\title{
THE PRISM MANIFOLD REALIZATION PROBLEM II
}

\author{
WILLIAM BALLINGER, YI NI, TYNAN OCHSE, AND FARAMARZ VAFAEE
}

\begin{abstract}
We continue our study of the realization problem for prism manifolds. Every prism manifold can be parametrized by a pair of relatively prime integers $p>1$ and $q$. We determine a complete list of prism manifolds $P(p, q)$ that can be realized by positive integral surgeries on knots in $S^{3}$ when $q>p$. The methodology undertaken to obtain the classification is similar to that of the case $q<0$ in an earlier paper.
\end{abstract}

\section{INTRODUCTION}

This paper is a continuation of $\left[\mathrm{BHM}^{+} 16\right]$, where the authors studied the Dehn surgery realization problem of prism manifolds. Recall that prism manifolds are spherical three-manifolds with dihedral type fundamental groups. Alternatively, an oriented prism manifold $P(p, q)$ has Seifert invariants

$$
(-1 ;(2,1),(2,1),(p, q)),
$$

where $q$ and $p>1$ are relatively prime integers. A surgery diagram of $P(p, q)$ is depicted in Figure $1 \mathrm{~A}$. When $q<0$, the realization problem for prism manifolds was solved in $\left[\mathrm{BHM}^{+} 16\right]$. More precisely, a complete list of $P(p, q)$, with $q<0$, that can be obtained by positive Dehn surgery on knots in $S^{3}$ is tabulated in $\left[\mathrm{BHM}^{+} 16\right.$, Table 1]. Indeed, every manifold in the table can be obtained by surgery on a Berge-Kang knot [BK]. Our main result, Theorem 1.1 below, provides the solution for those $P(p, q)$ with $q>p$ : see Table 1 .

Theorem 1.1. Given a pair of relatively prime integers $p>1$ and $q>p$, the prism manifold $P(p, q)$ can be obtained by $4 q$-surgery on a knot $K \subset S^{3}$ if and only if $P(p, q)$ belongs to one of the six families in Table 1. Moreover, in this case, there exists a Berge-Kang knot $K_{0}$ such that $P(p, q) \cong S_{4 q}^{3}\left(K_{0}\right)$, and that $K$ and $K_{0}$ have isomorphic knot Floer homology groups.

The methodology used to obtain Table 1 is similar to that of [Gre13, $\left.\mathrm{BHM}^{+} 16\right]$. When $q>p$, the prism manifold $P(p, q)$ bounds a negative definite four-manifold $X=X(p, q)$ with a Kirby diagram as in Figure 1D: see Section 2. Let $P(p, q)$ arise from surgery on a knot $K \subset S^{3}$. Let also $W_{4 q}=W_{4 q}(K)$ be the corresponding two-handle cobordism obtained by attaching a two-handle to the four-ball along the knot $K$ with framing $4 q$. Form the four-manifold $Z:=X \cup_{P(p, q)}\left(-W_{4 q}\right)$. It follows that $Z$ is a smooth, closed, negative definite four-manifold with $b_{2}(Z)=n+2$ for some $n \geq 1$ : see Figure 1D. Now, the celebrated theorem of Donaldson ("Theorem A") implies that the intersection pairing on $H_{2}(Z)$ is isomorphic to $-\mathbb{Z}^{n+2}$ [Don83], the Euclidean integer lattice with the negation of its usual dot product. This provides a necessary condition for $P(p, q)$ to be positive integer surgery on a knot; namely, the 
lattice $C(p, q)$, specified by the negative of the intersection pairing on $H_{2}(X)$, must embed as a codimension one sublattice of $\mathbb{Z}^{n+2}$. The key idea we use to sharpen this into a necessary and sufficient condition is the work of Greene [Gre13], which is built mainly on the use of the correction terms in Heegaard Floer homology in tandem with Donaldson's theorem. In order to state his theorem, we first require a combinatorial definition.

Definition 1.2. A vector $\sigma=\left(\sigma_{0}, \sigma_{1}, \ldots, \sigma_{n+1}\right) \in \mathbb{Z}^{n+2}$ that satisfies $0 \leq \sigma_{0} \leq \sigma_{1} \leq \cdots \leq$ $\sigma_{n+1}$ is a changemaker vector if for every $k$, with $0 \leq k \leq \sigma_{0}+\sigma_{1}+\cdots+\sigma_{n+1}$, there exists a subset $S \subset\{0,1, \ldots, n+1\}$ such that $k=\sum_{i \in S} \sigma_{i}$.

Using Lemma 2.6, the following is immediate from [Gre15, Theorem 3.3].

Theorem 1.3. Suppose $P(p, q)$ with $q>p$ arises from positive integer surgery on a knot in $S^{3}$. The lattice $C(p, q)$ is isomorphic to the orthogonal complement $(\sigma)^{\perp}$ of some changemaker vector $\sigma \in \mathbb{Z}^{n+2}$.

By determining the pairs $(p, q)$ which pass the embedding restriction of Theorem 1.3, we get the list of all prism manifolds $P(p, q)$ with $q>p$ that can possibly be realized by integer surgery on a knot in $S^{3}$ : again, see Table 1 . We still need to verify that every manifold in our list is indeed realized by a knot surgery. In fact, this is the case.

Theorem 1.4. Given a pair of relatively prime integers $p>1$ and $q>p, C(p, q) \cong(\sigma)^{\perp}$ for a changemaker vector $\sigma \in \mathbb{Z}^{n+2}$ if and only if $P(p, q)$ belongs to one of the six families in Table 1. Moreover, in this case, there exist a knot $K \subset S^{3}$ with $S_{4 q}^{3}(K) \cong P(p, q)$ and an isomorphism of lattices

$$
\varphi:\left(\mathbb{Z}^{n+2}, I\right) \rightarrow\left(H_{2}(Z),-Q_{Z}\right)
$$

such that $\varphi(\sigma)$ is a generator of $H_{2}\left(-W_{4 q}\right)$. Here I denotes the standard inner product on $\mathbb{Z}^{n+2}$ and $Q_{Z}$ is the intersection form of $Z=X(p, q) \cup\left(-W_{4 q}\right)$.

Remark 1.5. Theorem 1.4, in particular, highlights that the families in Table 1 are divided so that each changemaker vector corresponds to a unique family. However, a prism manifold $P(p, q)$ may belong to more than one family in Table 1 . We will address the overlaps between the families of Table 1 in Section 9: see Table 2.

Table 2 in $\left[\mathrm{BHM}^{+} 16\right]$ gives a conjecturally complete list of prism manifolds $P(p, q)$ with $q>0$ that can be obtained by performing surgery on a knot in $S^{3}$. Every manifold in $\left[\mathrm{BHM}^{+} 16\right.$, Table 2] is obtained by integral surgery on a Berge-Kang knot (see $\left[\mathrm{BHM}^{+} 16\right.$, Table 4] and [BK]). Theorem 1.1 proves $\left[\mathrm{BHM}^{+} 16\right.$, Conjecture 1.6] for the case $q>p$ since the manifolds in Table 1 coincide with those in $\left[\mathrm{BHM}^{+} 16\right.$, Table 2] with $q>p$. We leave open the realization problem for prism manifolds $P(p, q)$ with $0<q<p$. We plan to address this case in a future paper.

1.1. Organization. Section 2 collects the topological background on prism manifolds, and also reviews the essentials needed to prove our main results. In Section 3, we study $\mathrm{C}$-type lattices $C(p, q)$ that are central in the present work. To prove Theorem 1.4, we begin with a study of the changemaker lattices (Section 4), i.e. lattices of the form $(\sigma)^{\perp} \subset \mathbb{Z}^{n+2}$ for some changemaker vector $\sigma \in \mathbb{Z}^{n+2}$. We then study when a changemaker lattice, with a standard 
basis, is isomorphic to a $\mathrm{C}$-type lattice, with its distinguished vertex basis. The key to answering this combinatorial question is detecting the irreducible elements in either of the lattices. Indeed, the standard basis elements of a changemaker lattice are irreducible (Lemma 4.4), as are the vertex basis elements of a $\mathrm{C}$-type lattice. Furthermore, the classification of the irreducible elements of $\mathrm{C}$-type lattices is given in Proposition 3.2. We collect many structural results about these lattices in Sections 3 and 4.

We classify the changemaker $\mathrm{C}$-type lattices based on how $x_{0}$, the first element in the ordered basis of a $\mathrm{C}$-type lattice, is written in terms of the standard orthonormal basis elements of $\mathbb{Z}^{n+2}$. Accordingly, Sections 5, 6, and 7 will enumerate the possible changemaker vectors whose orthogonal complements are $\mathrm{C}$-type lattices. Section 8 tabulates the corresponding prism manifolds.

Finally, in Section 9, we address the overlaps between the families in Table 1. More precisely, we provide distinct knots corresponding to distinct changemakers that result in the same prism manifold. We then proceed with proving Theorems 1.1 and 1.4.

Acknowledgements. The heart of this project was done during the Caltech's Summer Undergraduate Research Fellowships (SURF) program in the summer of 2017. Y. N. was partially supported by NSF grant number DMS-1252992 and an Alfred P. Sloan Research Fellowship. F. V. was partially supported by an AMS-Simons Travel Grant. W. B. would like to thank William H. and Helen Lang, as well as Samuel P. and Frances Krown, for their generous support through the SURF program. T. O. would like to thank Joanna Wall Muir and Mr. James Muir for their generous support through the SURF program.

\section{Preliminaries}

For a pair of relatively prime integers $p>1$ and $q$, the prism manifold $P(p, q)$ is a Seifert fibered space with a surgery description depicted in Figure 1A. It is shown in $\left[\mathrm{BHM}^{+} 16\right]$ that if $P(p, q)$ is obtained by surgery on a knot in $S^{3}, p$ must be odd.

An equivalent surgery description for $P(p, q)$ is depicted in Figure 1D. To get the coefficients $a_{i}$, write $\frac{2 q-p}{q-p}$ in a Hirzebruch-Jung continued fraction

$$
\frac{2 q-p}{q-p}=a_{1}-\frac{1}{a_{2}-\frac{1}{\ddots \cdot-\frac{1}{a_{n}}}}=\left[a_{1}, a_{2}, \ldots, a_{n}\right]^{-} .
$$

From this point on in the paper, we assume that $q>p$. As a result, we have $a_{1} \geq 3$ in Equation (1). Moreover, each $a_{i} \geq 2$.

Definition 2.1. The $C$-type lattice $C(p, q)$ has a basis

$$
\left\{x_{0}, \ldots, x_{n}\right\},
$$




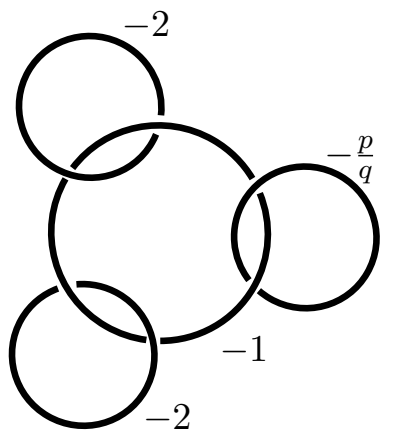

A.

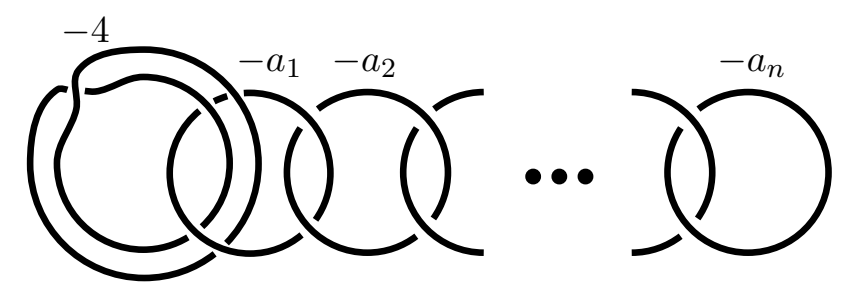

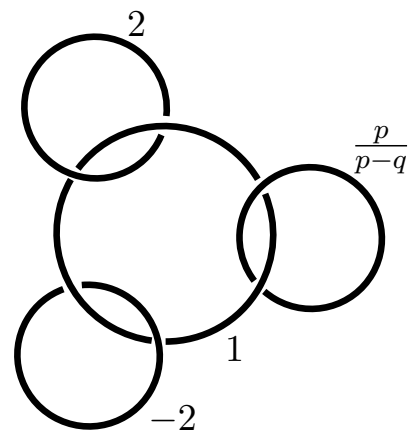

B.

C.

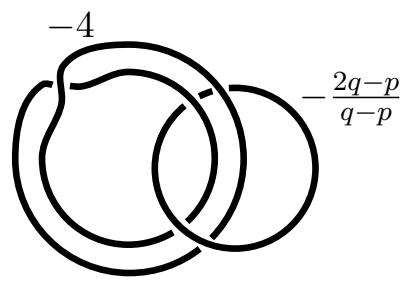

D.

Figure 1. Surgery presentations of $P(p, q)$. A and B correspond to the two equivalent choices of Seifert invariants $(-1 ;(2,1),(2,1),(p, q))$ and $(1 ;(2,1),(2,-1),(p, q-p))$. To go from B to C, blow down two 1-framed unknots in sequence: first blow down the middle unknot, changing the framing on the upper left unknot to 1 , and then blow down the upper left unknot. Finally, to get to $\mathrm{D}$, use slam-dunk moves to expand $\frac{2 q-p}{q-p}$ in a continued fraction. The last link gives a negative-definite four-manifold if $q<0$ or $q>p$.

and inner product given by

$$
\left\langle x_{i}, x_{j}\right\rangle= \begin{cases}4 & i=j=0 \\ a_{i} & i=j>0 \\ -2 & \{i, j\}=\{0,1\} \\ -1 & |i-j|=1, i>0, j>0 \\ 0 & |i-j|>1,\end{cases}
$$

where the coefficients $a_{i}$, for $i \in\{1, \cdots, n\}$, are defined by the continued fraction (1). We call (2) the vertex basis of $C(p, q)$. 


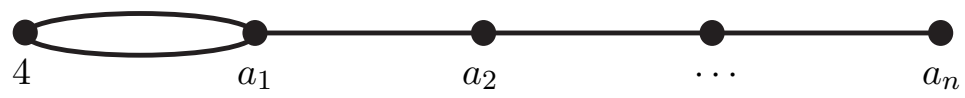

Figure 2. A C-type lattice $C(p, q)$ with $\frac{2 q-p}{q-p}=\left[a_{1}, a_{2}, \cdots, a_{n}\right]^{-}$. Note that $a_{1} \geq 3$ when $q>p$.

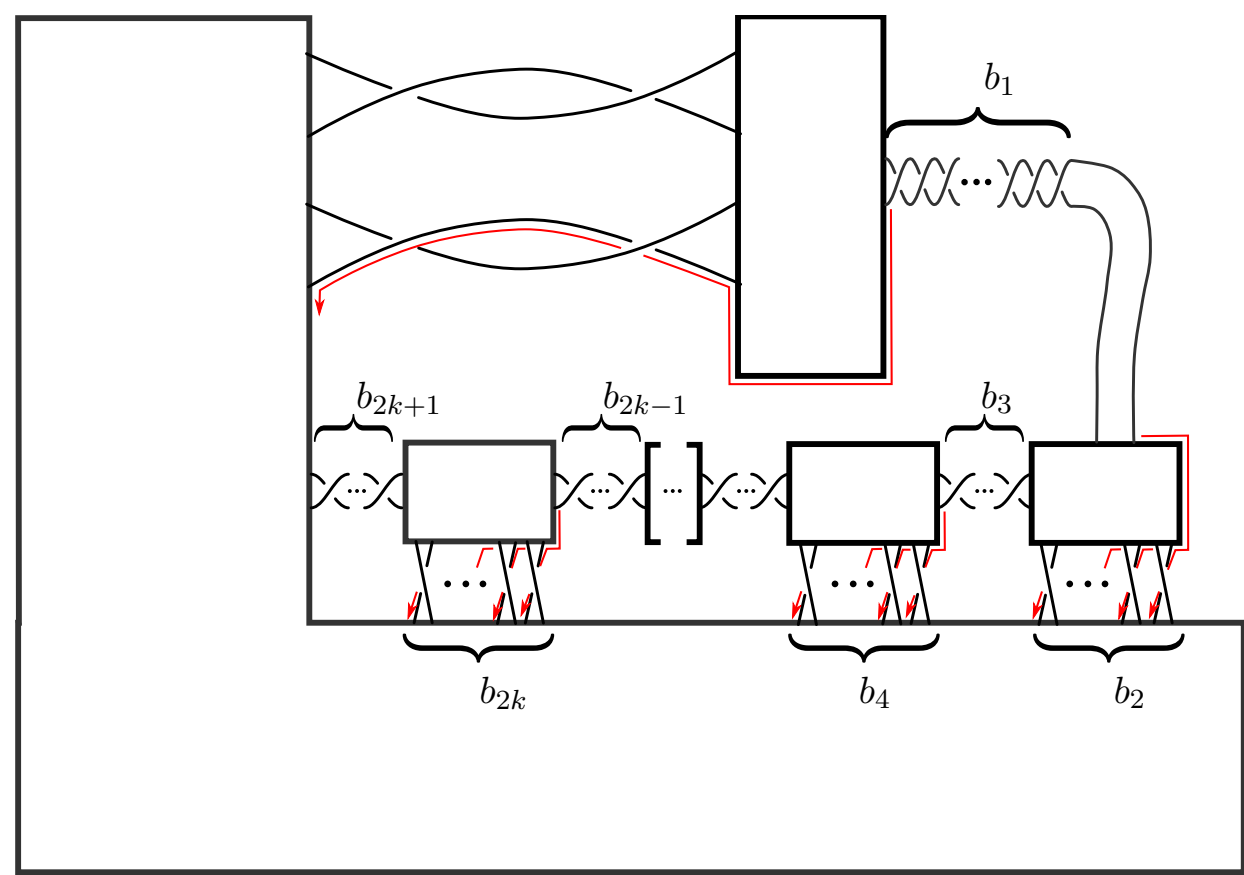

Figure 3. A handle decomposition of a surface embedded in $S^{3}$. The boundary of this surface is an alternating Montesinos link whose branched double cover is $P(p, q)$, and the branched double cover of $B^{4}$ over this surface with its interior pushed into the interior of $B^{4}$ is $X(p, q)$. Sliding the 1 -handles in this picture along the red arrows and then cancelling all but one of the 0 handles gives Figure 5 . This surface depends on parameters $b_{1}, \ldots, b_{m}$ where $m$ is either $2 k+1$ or $2 k$; if $m=2 k$ omit the band labelled $b_{2 k+1}$.

Let $X=X(p, q)$ be the four-manifold, bounded by $P(p, q)$, with a Kirby diagram as depicted in Figure 1D. The inner product space $\left(H_{2}(X),-Q_{X}\right)$ equals $C(p, q)$, where $Q_{X}$ denotes the intersection pairing of $X$ : see Figure 2. Note that $b_{2}(X)=n+1$, where $n$ is defined in (1).

Remark 2.2. When $q<0$ in Equation (1), it follows that $a_{1}=2$ and $C(p, q)$ is indeed isomorphic to a D-type lattice $\left[\mathrm{BHM}^{+} 16\right.$, Definition 2.8]. The prism manifold realization problem is solved in this case $\left[\mathrm{BHM}^{+} 16\right]$.

2.1. The four-manifold $X(p, q)$ revisited. In this subsection, we present a different construction of the four-manifold $X(p, q)$ as the branched double cover of $B^{4}$ over a particular surface: see Figure 3. As a Seifert fibered rational homology sphere, the prism manifold 


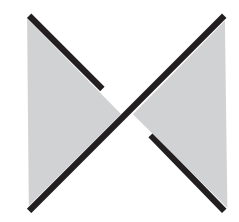

FiguRE 4. The coloring convention

$P(p, q)$ is the branched double cover of $S^{3}$ branched along a Montesinos link [Mon73]: choose $b_{1}, \ldots, b_{n}$ so that

$$
\frac{p}{q-p}=b_{1}+\frac{1}{b_{2}+\frac{1}{\ddots \cdot+\frac{1}{b_{m}}}}=\left[b_{1}, b_{2}, \ldots, b_{m}\right]^{+} .
$$

Since $q>p, \frac{p}{q-p}>0$ and we can choose the $b_{i}$ so that $b_{1} \geq 0$ and $b_{i}>0$ for $i>1$. The boundary of the surface $\Sigma$ drawn in Figure 3 is an alternating Montesinos link $L$, and $\Sigma$ itself is the surface formed by the black regions in a checkerboard coloring of the alternating diagram. We point out that we are using the coloring convention as in Figure 4. The branched double cover of $S^{3}$ branched along $L$ is $P(p, q)$. Let $X_{\Sigma}$ be the branched double cover of $B^{4}$ over the surface $\Sigma$ with its interior pushed into the interior of $B^{4}$. With this notation in place:

Proposition 2.3. $X(p, q) \cong X_{\Sigma}$.

We first recall the following lemma that will be used in the proof of Proposition 2.3 and also in Section 8.

Lemma 2.4 (Lemma 9.5 (1) and (3) of [Gre13]). For integers $r, s, t \geq 0$,

1. $\left[\ldots, r, 2^{[s]}, t, \ldots\right]^{-}=[\ldots, r-1,-(s+1), t-1, \ldots]^{-}$, and

2. $\left[\ldots, s, 2^{[t]}\right]^{-}=[\ldots, s-1,-(t+1)]^{-}$,

where $2^{[a]}$ means that the entry 2 appears a times.

We now proceed to prove Proposition 2.3. In order to obtain a Kirby diagram of branched double covers, we closely follow the treatment of [AK80]; in particular, see [AK80, Figure 4].

Proof of Proposition 2.3. Figure 3 depicts a handle decomposition of the surface $\Sigma$ whose branched double cover is $X_{\Sigma}$. By sliding the 1-handles along the red arrows in Figure 3 and then canceling all but only one of the 0-handles, we obtain the surface in Figure 5: a disc with several bands attached. The odd-numbered $b_{2 i+1}$ with $0<i<\frac{m-1}{2}$ contribute bands with $b_{2 i+1}+2$ half-twists, $b_{1}$ contributes a band with $b_{1}+3$ half-twists, and $b_{m}$ contributes a band with $b_{m}+1$ half-twists when $m$ is odd. The even-numbered $b_{2 i}$ contribute $b_{2 i}-1$ bands each, each with 2 half-twists. Therefore, the coefficients $a_{1}, \ldots, a_{n}$ of Figure 6 are

$$
\left(a_{1}, \ldots, a_{n}\right)= \begin{cases}\left(b_{1}+3,2^{\left[b_{2}-1\right]}, b_{3}+2,2^{\left[b_{4}-1\right]}, \ldots, 2^{\left[b_{m-1}-1\right]}, b_{m}+1\right) & m \text { odd } \\ \left(b_{1}+3,2^{\left[b_{2}-1\right]}, b_{3}+2,2^{\left[b_{4}-1\right]}, \ldots, b_{m-1}+2,2^{\left[b_{m}-1\right]}\right) & m \text { even }\end{cases}
$$




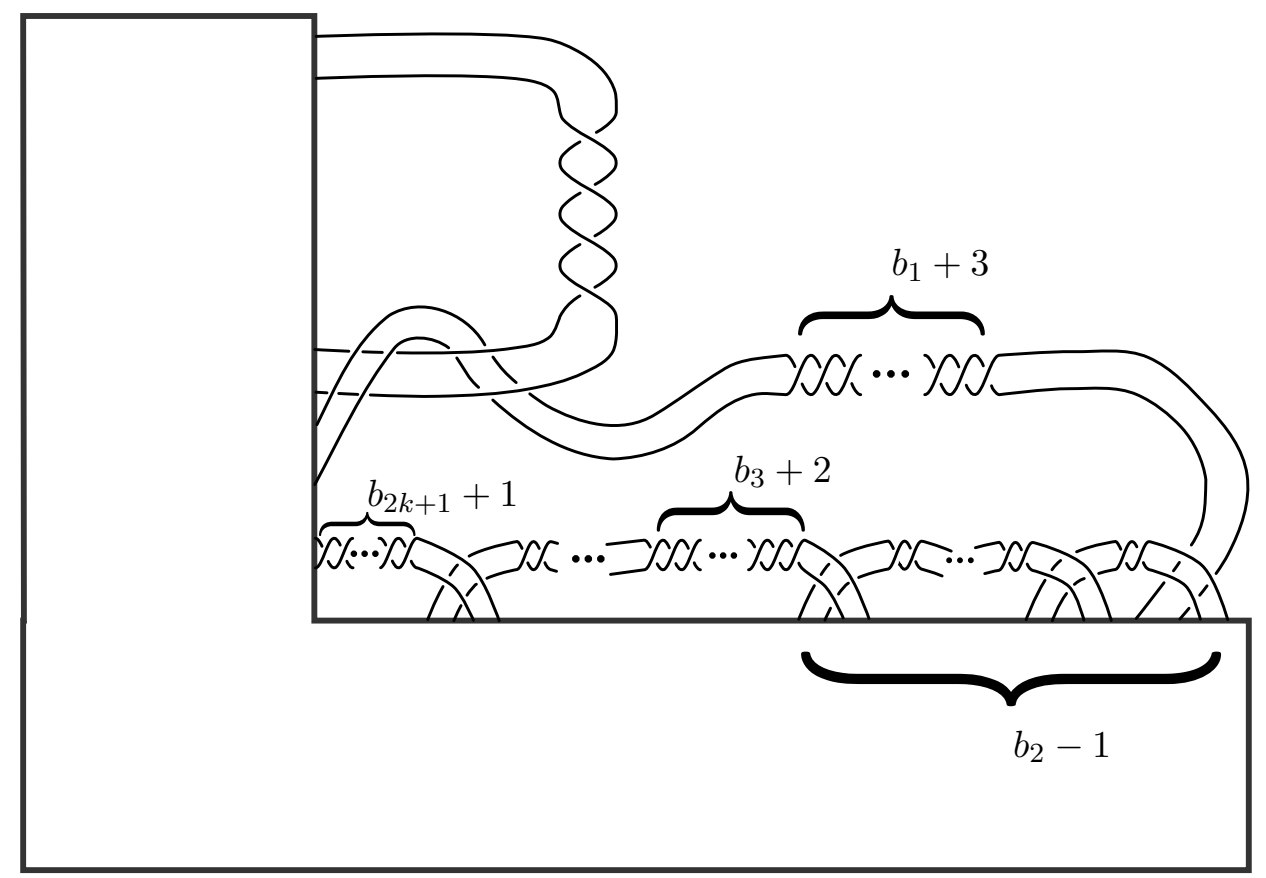

FiguRE 5. Another view of the surface shown in Figure 3. From this picture a Kirby diagram representing the branched double cover of $B^{4}$ over this surface (shown in Figure 6) can be read off using the methods of Figure 4 in [AK80]. As before, if $m$ is even omit the band labelled $b_{2 k+1}$.

Using Lemma 2.4,

$$
\begin{aligned}
{\left[a_{1}, \ldots, a_{n}\right]^{-} } & =\left[b_{1}+2,-b_{2}, b_{3},-b_{4}, \ldots, \pm b_{m}\right]^{-} \\
& =\left[b_{1}+2, b_{2}, \ldots, b_{m}\right]^{+} \\
& =\frac{p}{q-p}+2 \\
& =\frac{2 q-p}{q-p}
\end{aligned}
$$

That is, the $a_{i}$ in Equation (4) are the same as those of Equation (1). The branched double cover of $B^{4}$ branched over the surface in Figure 5 is depicted in Figure 6; comparing it with Figure 1D, the result follows.

2.2. Input from Heegaard Floer homology. We assume familiarity with Floer homology and only review the essential input here for completeness. See, for instance, [OS04a, OS04b]. In [OS03], Ozsváth and Szabó defined the correction term $d(Y, \mathfrak{t})$ that associates a rational number to an oriented rational homology sphere $Y$ equipped with a $\operatorname{Spin}^{c}$ structure $t$. If $Y$ is boundary of a negative definite four-manifold $X$, then

$$
c_{1}(\mathfrak{s})^{2}+b_{2}(X) \leq 4 d(Y, \mathfrak{t})
$$




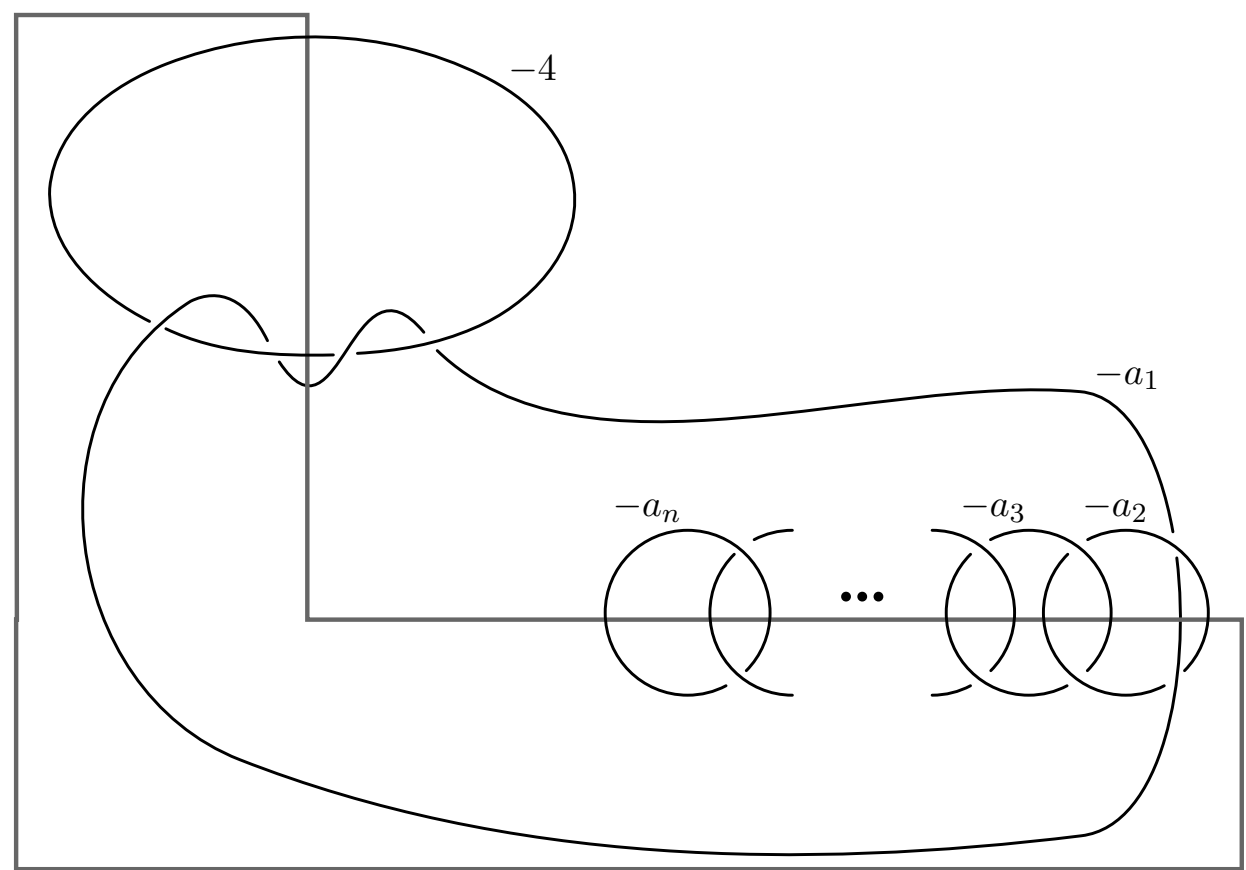

FiguRE 6. A Kirby diagram representing the branched double cover of the surface in Figure 3. This is the same as the diagram defining $X(p, q)$. The grey box is not part of the link, but is included only to show the relationship with Figure 5.

for any $\mathfrak{s} \in \operatorname{Spin}^{c}(X)$ that extends $\mathfrak{t} \in \operatorname{Spin}^{c}(Y)$.

Definition 2.5. A smooth, compact, negative definite four-manifold $X$ is sharp if for every $\mathfrak{t} \in \operatorname{Spin}^{c}(Y)$, there exists some $\mathfrak{s} \in \operatorname{Spin}^{c}(X)$ extending $\mathfrak{t}$ such that the equality is realized in Equation (5).

Using Proposition 2.3, the following is immediate from [OS05b, Theorem 3.4].

Lemma 2.6. $X(p, q)$ is a sharp four-manifold.

2.3. Alexander polynomials of knots on which surgery yield $P(p, q)$ with $q>p$. Using techniques that will be developed in the next sections in tandem with Theorem 1.3, we will find the classification of all C-type lattices $C(p, q)$ that are isomorphic to $(\sigma)^{\perp}$ for some changemaker vector $\sigma$ in $\mathbb{Z}^{n+2}$. If the corresponding prism manifold $P(p, q)$ is indeed arising from surgery on a knot $K \subset S^{3}$, we are able to compute the Alexander polynomial of $K$ from the values of the components of $\sigma$ : let $S$ be the closed surface obtained by capping off a Seifert surface for $K$ in $W_{4 q}$. It is straightforward to check that the class $[S]$ generates $H_{2}\left(W_{4 q}\right)$. It follows from Theorem 1.3 that, under the embedding $H_{2}(X) \oplus H_{2}\left(-W_{4 q}\right) \hookrightarrow H_{2}(Z)$, the homology class $[S]$ gets mapped to a changemaker vector $\sigma$. Let $\left\{e_{0}, e_{1}, \cdots, e_{n+1}\right\}$ be the 
standard orthonormal basis for $\mathbb{Z}^{n+2}$, and write

$$
\sigma=\sum_{i=0}^{n+1} \sigma_{i} e_{i}
$$

Also, define the characteristic covectors of $\mathbb{Z}^{n+2}$ to be

$$
\operatorname{Char}\left(\mathbb{Z}^{n+2}\right)=\left\{\sum_{i=0}^{n+1} \mathfrak{c}_{i} e_{i} \mid \mathfrak{c}_{i} \text { odd for all } i\right\} .
$$

We remind the reader that, writing the Alexander polynomial of $K$ as

$$
\Delta_{K}(T)=b_{0}+\sum_{i>0} b_{i}\left(T^{i}+T^{-i}\right)
$$

the $k$-th torsion coefficient of $K$ is

$$
t_{k}(K)=\sum_{j \geq 1} j b_{k+j}
$$

where $k \geq 0$. The following lemma is immediate from [Gre15, Lemma 2.5].

Lemma 2.7. The torsion coefficients satisfy

$$
t_{i}(K)=\left\{\begin{array}{cl}
\min _{\mathfrak{c}} \frac{\mathfrak{c}^{2}-n-2}{8}, & \text { for each } i \in\{0,1, \ldots, 2 q\}, \\
0, & \text { for } i>2 q .
\end{array}\right.
$$

where $\mathfrak{c}$ is subject to

$$
\mathfrak{c} \in \operatorname{Char}\left(\mathbb{Z}^{n+2}\right), \quad\langle\mathfrak{c}, \sigma\rangle+4 q \equiv 2 i \quad(\bmod 8 q)
$$

And for $i>0$,

$$
b_{i}=t_{i-1}-2 t_{i}+t_{i+1}, \quad \text { for } i>0,
$$

and

$$
b_{0}=1-2 \sum_{i>0} b_{i}
$$

where the $b_{i}$ are as in (6).

\section{C-Type Lattices}

This section assembles facts about C-type lattices that will be used in the classification. We mainly use the notation of [Gre13, $\left.\mathrm{BHM}^{+} 16\right]$. Recall that we always assume $q>p$, so $a_{1} \geq 3$ : see Figure 2.

Let $L$ be a lattice. Given $v \in L$, let $|v|=\langle v, v\rangle$ be the norm of $v$. An element $\ell \in L$ is reducible if $\ell=x+y$ for some nonzero $x, y \in L$, with $\langle x, y\rangle \geq 0$, and irreducible otherwise. An element $\ell \in L$ is breakable if $\ell=x+y$ with $|x|,|y| \geq 3$ and $\langle x, y\rangle=-1$, and unbreakable otherwise.

Among the irreducible elements of a lattice, intervals are the most convenient for us: 
Definition 3.1. In a C-type lattice, if $I$ is any subset of $\left\{x_{0}, x_{1}, \ldots, x_{n}\right\}$ then write $[I]=$ $\sum_{x \in A} x$. An interval is an element of the form $[I]$ with $I=\left\{x_{a}, x_{a+1}, \ldots, x_{b}\right\}$ for $0 \leq a \leq$ $b \leq n$. We say that $a$ is the left endpoint of the interval, and $b$ is the right endpoint of the interval. Say that $[I]$ contains $x_{i}$ if $I$ does.

Given the fact that $a_{1} \geq 3$, the following is immediate from [Gre13, Proposition 3.3].

Proposition 3.2. If $v \in C(p, q)$ is irreducible, $v=\epsilon[I]$ for some $\epsilon= \pm 1$ and $[I]$ an interval.

Definition 3.3. Given a lattice $L$ and a subset $V \subset L$, the pairing graph is $\hat{G}(V)=(V, E)$, where $e=\left(v_{i}, v_{j}\right) \in E$ if $\left\langle v_{i}, v_{j}\right\rangle \neq 0$.

Corollary 3.4. The lattice $C(p, q)$ is indecomposable; that is, $C(p, q)$ is not the direct sum of two nontrivial lattices.

Proof. Suppose that $C(p, q) \cong L_{1} \oplus L_{2}$. Then each $x_{i}$, being irreducible, must be in either $L_{1}$ or $L_{2}$. However, any element of $L_{1}$ has zero pairing with any element of $L_{2}$. Since $\left\langle x_{i}, x_{i+1}\right\rangle \neq 0, \hat{G}\left(\left\{x_{0}, \ldots, x_{n}\right\}\right)$ is connected. This means that all of the $x_{i}$ are in the same part of the decomposition, and the other is trivial.

In a C-type lattice, we have that $\left|\left\langle x_{0}, x_{1}\right\rangle\right|=2$. It turns out that the inner product of $x_{0}$ with any other element in the $C$-type lattice lives in $2 \mathbb{Z}$. The following lemma is straightforward to prove.

Lemma 3.5. For any $v \in C(p, q),\left\langle x_{0}, v\right\rangle$ is even. In particular, the reflection $r_{x_{0}}: v \mapsto$ $v-2 \frac{\left\langle x_{0}, v\right\rangle}{\left\langle x_{0}, x_{0}\right\rangle} x_{0}$ about $x_{0}^{\perp}$ is an involution of $C(p, q)$.

Definition 3.6. A vertex $x_{i}$ has high weight if $i>0$ and $\left|x_{i}\right|=a_{i}>2$.

Proposition 3.7. An element $\epsilon[I] \in C(p, q)$ with $\epsilon \in\{ \pm 1\}$ is unbreakable if and only if $[I]$ contains at most one element of high weight.

Proof. The conclusion is obvious when $I=\left\{x_{0}\right\}$. Now we assume $I \neq\left\{x_{0}\right\}$. If $[I]$ does not contain $x_{0}$, this reduces to the analogous fact about linear lattices [Gre13, Corollary $3.5(4)$ ]. The reflection $r_{x_{0}}$ exchanges intervals with left endpoint 0 and intervals with left endpoint 1 , which reduces the case of intervals containing $x_{0}$ to the case of intervals not containing $x_{0}$.

Definition 3.8. Consider the graph $C$ on vertex set $\left\{x_{0}, \ldots, x_{n}\right\}$ that has two edges between $x_{0}$ and $x_{1}$ and one edge between $x_{i}$ and $x_{i+1}$ for $0<i<n$. Given two intervals $[I]$ and $[J]$, say that an edge of $C$ is dangling if one of its ends is in $I$, the other is in $J$, and at least one of the ends is not in $I \cap J$. Write $\delta([I],[J])$ for the number of dangling edges.

Lemma 3.9. For two intervals $[I],[J],\langle[I],[J]\rangle=|[I \cap J]|-\delta([I],[J])$.

Proof. Suppose $I=\left\{x_{a}, \ldots, x_{b}\right\}$ and $J=\left\{x_{c}, \ldots, x_{d}\right\}$. Then we can express

$$
\langle[I],[J]\rangle=\sum_{i=a}^{b} \sum_{j=c}^{d}\left\langle x_{i}, x_{j}\right\rangle
$$


Terms in this sum with $|i-j|>1$ vanish. The remaining terms either have $x_{i}$ and $x_{j}$ in $I \cap J$, so occur as terms in the expansion of $|[I \cap J]|$, or have at least one of $x_{i}$ or $x_{j}$ not in $I \cap J$, so contribute to $\delta([I],[J])$.

We frequently use the following lemma, which is stated without proof.

Lemma 3.10. Let $I \neq\left\{x_{0}\right\}$ be an interval. Then

$$
|[I]|=2+\sum_{x_{i} \in I \backslash\left\{x_{0}\right\}}\left(\left|x_{i}\right|-2\right) .
$$

Given the structure of a C-type lattice, the following is immediate.

Lemma 3.11. For any intervals $I, J, \delta([I],[J])$ is $0,1,2$, or 3 . If $\delta([I],[J])=3$, then $\left\langle x_{0},[I]\right\rangle=-\left\langle x_{0},[J]\right\rangle= \pm 2$.

To more precisely describe the value $\delta([I],[J])$, it will be convenient to use some terminology from [Gre13]:

Definition 3.12. For two intervals $[I]$ and $[J]$ with left endpoints $i_{0}, j_{0}$ and right endpoints $i_{1}, j_{1}$, say that $[I]$ and $[J]$ are distant if either $i_{1}+1<j_{0}$ or $j_{1}+1<i_{0}$, that $[I]$ and $[J]$ share a common end if $i_{0}=j_{0}$ or $i_{1}=j_{1}$, and that $[I]$ and $[J]$ are consecutive if $i_{1}+1=j_{0}$ or $j_{1}+1=i_{0}$. Write $[I] \prec[J]$ if $I \subset J$ and $[I]$ and $[J]$ share a common end, and $[I] \dagger[J]$ if they are consecutive. If $[I]$ and $[J]$ are either consecutive or share a common end, say that they abut. If $I \cap J$ is nonempty and $[I]$ and $[J]$ do not share a common end, write $[I] \pitchfork[J]$.

Remark 3.13. If $\left\langle[I], x_{0}\right\rangle=\left\langle[J], x_{0}\right\rangle$ or if either $\left\langle[I], x_{0}\right\rangle$ or $\left\langle[J], x_{0}\right\rangle$ is zero, then $\delta([I],[J])$ is 0 if $[I]$ and $[J]$ are distant, 1 if $[I]$ and $[J]$ abut, and 2 if $[I] \pitchfork[J]$. If $\left\langle[I], x_{0}\right\rangle \neq\left\langle[J], x_{0}\right\rangle$ and both are nonzero, $\delta([I],[J])$ is 2 if $[I]$ and $[J]$ abut, and 3 if $[I] \pitchfork[J]$. In the latter case, $[I]$ and $[J]$ are never distant.

We will also need to know which irreducible elements of $C(p, q)$ are breakable. In light of Proposition 3.2, we only need to study that for intervals.

Lemma 3.14 (Lemma 3.10 of $\left[\mathrm{BHM}^{+} 16\right]$ ). An interval $[A]$ is breakable if there are at least two high weight vertices.

Definition 3.15. For an unbreakable interval $\left[I_{j}\right] \in C(p, q)$ with $\left|\left[I_{j}\right]\right| \geq 3$, let $x_{z_{j}}$ be the unique element with $\left|x_{z_{j}}\right| \geq 3$.

We end this section by determining when two C-type lattices are isomorphic.

Proposition 3.16. If $C(p, q) \cong C\left(p^{\prime}, q^{\prime}\right)$, then $p=p^{\prime}$ and $q=q^{\prime}$.

Proof. If $L$ is a lattice isomorphic to $C(p, q)$, then to recover $p$ and $q$ from $L$ it suffices to recover the ordered sequence of norms $\left(\left|x_{1}\right|,\left|x_{2}\right|, \cdots,\left|x_{n}\right|\right)$. To do this, we will first identify the elements of this sequence that are at least 3, and then fill in the 2 's.

We claim that unless $(p, q)=(2,3)$, there is a unique (up to sign) unbreakable irreducible element $y$ such that $|y|=4$ and $\langle y, v\rangle$ is even for all $v$ in $L$, and $y= \pm x_{0}$. Let $I \neq\left\{x_{0}\right\}$ 
be any interval representing an unbreakable irreducible element with norm 4 . Suppose $I=$ $\left\{x_{a}, x_{a+1}, \ldots, x_{b}\right\}$. If $a>1$, then $\left\langle[I], x_{a-1}\right\rangle=-1$ is odd. If $b<n$, then $\left\langle[I], x_{b+1}\right\rangle=-1$ is odd. So we assume $a=0$ or 1 , and $b=n$. If $I$ contains at least two high weight vertices, then $I$ is breakable. So $x_{1}$ is the only high weight vertex, and $4=|[I]|=\left|x_{1}\right|$. If $n>1$, then $\left\langle[I], x_{b}\right\rangle=1$ is odd. So $n=1,\left|x_{1}\right|=4$. From $(1)$ we get $(p, q)=(2,3)$.

From now on, we assume $(p, q) \neq(2,3)$. Let $R$ be the sublattice of $L$ generated by $x_{0}$ and all vectors of norm 2 . Since $L$ contains no vectors of norm 1 , any vector of norm 2 in $L$ is irreducible. By Lemma 3.10, then, $R$ is generated by $x_{0}$ and the $x_{i}$ with $\left|x_{i}\right|=2$.

Now, let $V_{0}$ be the set of irreducible, unbreakable elements of $L \backslash\left\{ \pm x_{0}\right\}$ with norm at least 3 , and let $V$ be the quotient of $V_{0}$ by the relation $v \sim u$ whenever either $v-u \in R$ or $v+u \in R$. Every element of $V_{0}$ corresponds to an interval containing a unique high-weight vertex, and $v \sim u$ if and only if these high-weight vertices are the same. Therefore, $V$ consists of precisely the equivalence classes of the $x_{i}$ with $\left|x_{i}\right| \geq 3, i>0$, and if $v \in V_{0}$ with $v \sim x_{i}$ we have $|v|=\left|x_{i}\right|$.

Finally, let $W$ be the set of indecomposible components of $R$, so each element of $W$ corresponds to either $x_{0}$ or a run of 2's in the sequence of norms $\left(\left|x_{1}\right|,\left|x_{2}\right|, \ldots,\left|x_{n}\right|\right)$. Let $\mathcal{B}$ be the bipartite graph with vertex set $V \cup W$, and an edge between $v \in V$ and $w \in W$ if there is a representative $\tilde{v} \in L$ of $v$ and an element $\tilde{w} \in W$ such that $\langle\tilde{v}, \tilde{w}\rangle=-1$, or $w$ corresponds to $x_{0}$ and $\left\langle\tilde{v}, x_{0}\right\rangle=-2$. Then $v$ and $w$ neighbor in $\mathcal{B}$ if and only if the element $x_{i}$ representing $v$ is adjacent to $x_{0}$ or the run of 2's corresponding to $w$, so $\mathcal{B}$ is in fact a path. Furthermore, there is a unique element $w_{0} \in W$ that contains $x_{0}$, and $w_{0}$ must be one of the ends of the path $\mathcal{B}$. We can now recover $\left(\left|x_{1}\right|,\left|x_{2}\right|, \ldots,\left|x_{n}\right|\right)$ as follows: The vertex $w_{0}$ neighbors a unique element $v \in V$ in $\mathcal{B}$. The rest of the sequence is completed in the following way - as we travel down the path $\mathcal{B}$, when we encounter an element $w \in W$ we add rk $w$-many 2 's to the sequence, and when we encounter an element $v \in V$ we add $|\tilde{v}|$ to the sequence for $\tilde{v}$ a representative of $v$.

\section{Changemaker Lattices}

A lattice is called a changemaker lattice if it is isomorphic to the orthogonal complement of a changemaker vector. Whenever $P(p, q)$, with $q>p$, comes from positive integer surgery on a knot, $C(p, q)$ is isomorphic to a changemaker lattice $(\sigma)^{\perp} \subset \mathbb{Z}^{n+2}$. In this section, we will assemble some basic structural results about C-type lattices that are isomorphic to changemaker lattices.

Write $\left(e_{0}, e_{1}, \ldots, e_{n+1}\right)$ for the orthonormal basis of $\mathbb{Z}^{n+2}$, and write $\sigma=\sum_{i} \sigma_{i} e_{i}$. Since $C(p, q)$ is indecomposable (Corollary 3.4), $\sigma_{0} \neq 0$, otherwise $(\sigma)^{\perp}$ would have a direct summand $\mathbb{Z}$. So $\sigma_{0}=1$.

We will need several results from [Gre13, Section 3] about changermaker lattices: 
Definition 4.1. The standard basis of $(\sigma)^{\perp}$ is the collection $S=\left\{v_{1}, \ldots, v_{n}\right\}$, where

$$
v_{j}=\left(2 e_{0}+\sum_{i=1}^{j-1} e_{i}\right)-e_{j}
$$

whenever $\sigma_{j}=1+\sigma_{0}+\cdots+\sigma_{j-1}$, and

$$
v_{j}=\left(\sum_{i \in A} e_{i}\right)-e_{j}
$$

whenever $\sigma_{j}=\sum_{i \in A} \sigma_{i}$, with $A \subset\{0, \ldots, j-1\}$ chosen to maximize the quantity $\sum_{i \in A} 2^{i}$. A vector $v_{j} \in S$ is called tight in the first case, just right in the second case as long as $i<j-1$ and $i \in A$ implies that $i+1 \in A$, and gappy if there is some index $i$ with $i \in A, i<j-1$, and $i+1 \notin A$. Such an index, $i$, is a gappy index for $v_{j}$.

The standard basis $S$ is in fact a basis of $C(p, q)$.

Definition 4.2. For $v \in \mathbb{Z}^{n+2}, \operatorname{supp} v=\left\{i \mid\left\langle e_{i}, v\right\rangle \neq 0\right\}$ and $\operatorname{supp}^{+} v=\left\{i \mid\left\langle e_{i}, v\right\rangle>0\right\}$.

Lemma 4.3 (Lemma 3.12 (3) in [Gre13]). If $\left|v_{k+1}\right|=2$, then $k$ is not a gappy index for any $v_{j}$ with $j \in\{1, \cdots, n+1\}$.

Lemma 4.4 (Lemma 3.13 in [Gre13]). Each $v_{j} \in S$ is irreducible.

Lemma 4.5 (Lemma 3.15 in [Gre13]). If $v_{j} \in S$ is breakable, then it is tight.

Lemma 4.6 (Lemma 3.14 (2) (3) in [Gre13]). Suppose that $v_{t} \in S$ is tight.

(1) If $v_{j}=e_{t}+e_{j-1}-e_{j}, j>t$, then $v_{t}+v_{j}$ is irreducible.

(2) If $v_{t+1}=e_{0}+e_{1}+\cdots+e_{t}-e_{t+1}$, then $v_{t+1}-v_{t}$ is irreducible.

Lemma 4.7 (Lemma 4.9 in $\left.\left[\mathrm{BHM}^{+} 16\right]\right)$. For any $v_{j} \in S$, we have $j-1 \in \operatorname{supp} v_{j}$.

For the rest of this section, suppose $\sigma=\left(\sigma_{0}, \sigma_{1}, \ldots, \sigma_{n+1}\right) \in \mathbb{Z}^{n+2}$ is a changemaker vector such that $(\sigma)^{\perp}$ is isomorphic to a C-type lattice $C(p, q)$ with $q>p$. Also, let $x_{0}, \ldots, x_{n}$ be the vertex basis of $C(p, q)$, and let $S=\left(v_{1}, \ldots, v_{n+1}\right)$ be the standard basis of $(\sigma)^{\perp}$. Each $v_{i}$ is an irreducible element in a C-type lattice (Lemma 4.4), so corresponds to some interval (Proposition 3.2). By a slight abuse of notation, denote $\left[v_{i}\right]$ for the interval corresponding to $v_{i}$. Let $\epsilon_{i} \in\{ \pm 1\}$ satisfy $v_{i}=\epsilon_{i}\left[v_{i}\right]$.

The C-type lattice $C(p, q)$ contains an element $x_{0}$ with $\left|x_{0}\right|=4$, and any vector of norm 4 in $\mathbb{Z}^{n+2}$ is of the form either $\pm 2 e_{k}$ or $\pm e_{k_{0}} \pm e_{k_{1}} \pm e_{k_{2}} \pm e_{k_{3}}$ for distinct indices $k_{i}$. Vectors of the first form cannot be in $(\sigma)^{\perp}$ since $\sigma_{0} \neq 0$, so $x_{0}$ must be of the second form. In fact, we can say a little bit more about how $x_{0}$ can be written in terms of the $e_{i}$. We start by the following lemma.

Lemma 4.8. There is no element $v \in C(p, q)$ with $\left\langle v, x_{0}\right\rangle \neq 0$ and $|v|=2$.

Proof. Since $C(p, q)$ is indecomposible, it contains no $x$ with $|x|=1$ (such an $x$ would generate a $\mathbb{Z}$-summand of $C(p, q))$. Therefore, if $v \in C(p, q)$ with $|v|=2$, it must be irreducible, so $v= \pm[I]$ for $[I]$ an interval. By Lemma 3.10, $[I]$ contains only $x_{0}$ or elements of norm 2 . In 
particular, $[I]$ does not contain $x_{1}$, since $a_{1} \geq 3$. This means that $[I]$ also cannot contain $x_{0}$, since then $[I]=x_{0}$ and $|v|=4$. Therefore, $\left\langle[I], x_{0}\right\rangle=0$, and so $\left\langle v, x_{0}\right\rangle=0$.

Proposition 4.9. For some indices $k_{1}<k_{2}<k_{3}, x_{0}$ is equal to one of $e_{0}+e_{k_{1}}+e_{k_{2}}-e_{k_{3}}$ or $e_{0}-e_{k_{1}}-e_{k_{2}}+e_{k_{3}}$, possibly after a global sign change in the isomorphism between $(\sigma)^{\perp}$ and $C(p, q)$.

Proof. Since $\left|x_{0}\right|=4$ and $x_{0} \in(\sigma)^{\perp}$,

$$
x_{0}=\delta_{0} e_{k_{0}}+\delta_{1} e_{k_{1}}+\delta_{2} e_{k_{2}}+\delta_{2} e_{k_{3}}
$$

for indices $k_{0}<k_{1}<k_{2}<k_{3}$ and signs $\delta_{i}$ such that $\sum_{i} \delta_{i} \sigma_{i}=0$. By a global sign change, we might as well assume that $\delta_{0}=1$. If $k_{0}>0,\left\langle x_{0}, v_{k_{0}}\right\rangle=-1$ is odd, violating Lemma 3.5. So $k_{0}=0$.

We claim that if $\sigma_{k_{i}}=\sigma_{k_{j}}$, then $\delta_{i}=\delta_{j}$. Otherwise $v=\delta_{i} e_{k_{i}}+\delta_{j} e_{k_{j}}$ would be in $(\sigma)^{\perp}$ with $|v|=2$ and $\left\langle v, x_{0}\right\rangle=2$, which contradicts Lemma 4.8. Therefore, if $\delta_{1}=-1$ then $\sigma_{1}>\sigma_{0}$, and so $\delta_{0} \sigma_{0}+\delta_{1} \sigma_{1}<0$. Therefore, $\delta_{2} \sigma_{2}+\delta_{3} \sigma_{3}>0$. Since $\sigma_{2} \leq \sigma_{3}$, this means that $\delta_{3}=1$, and then $\delta_{2}=-1$ since $\sigma_{1}<\sigma_{0}+\sigma_{2}+\sigma_{3}$. In the other case, if $\delta_{1}=1$ then $\delta_{0} \sigma_{0}+\delta_{1} \sigma_{1}>0$, so $\delta_{2} \sigma_{2}+\delta_{3} \sigma_{3}<0$ and $\delta_{3}=-1$. If also $\delta_{2}=-1$, then

$$
\sigma_{0}+\sigma_{1}=\sigma_{2}+\sigma_{3}
$$

Since $\sigma_{0} \leq \sigma_{1} \leq \sigma_{2} \leq \sigma_{3}$, this can only happen if all of the $\sigma_{i}$ are equal, again contradicting the fact that if $\sigma_{i}=\sigma_{j}$ we must have $\delta_{i}=\delta_{j}$.

Corollary 4.10. The vector $v_{1}$ is equal to $2 e_{0}-e_{1}$ if $k_{1}>1$, and $e_{0}-e_{1}$ otherwise. If $x_{0}=e_{0}-e_{k_{1}}-e_{k_{2}}+e_{k_{3}}$, the first of these occurs.

Proof. Note that $v_{1}$ is always either $e_{0}-e_{1}$ or $2 e_{0}-e_{1}$. Using Lemma 3.5, the first statement of the lemma follows. For the second statement, if $k_{1}=1$ and $v_{1}=e_{1}-e_{0}$, then if $x_{0}=e_{0}-e_{k_{1}}-e_{k_{2}}+e_{k_{3}}$ we have that $\left\langle v_{1}, x_{0}\right\rangle=2$ and $\left|v_{1}\right|=2$, contradicting Lemma 4.8.

Lemma 4.11. If $k_{1}>1, v_{1}$ is the only tight vector. If $k_{1}=1, v_{k_{2}}$ can be tight but there is no other tight vector.

Proof. We claim that if $v_{t}$ is tight, then either $t<k_{1}$ or $t=k_{2}$. Using Lemma 3.5, we must have that either $k_{2} \leq t<k_{3}$ or $t<k_{1}$ as otherwise $v_{t}$ will have odd pairing with $x_{0}$. If $k_{2}<t<k_{3}$, then

$$
\sigma_{t}=1+\sigma_{0}+\sigma_{1}+\cdots+\sigma_{t-1} \geq 1+\sigma_{0}+\sigma_{k_{1}}+\sigma_{k_{2}} .
$$

However, by Proposition 4.9, the fact that $\left\langle x_{0}, \sigma\right\rangle=0$ implies that

$$
\sigma_{k_{3}}=\sigma_{k_{2}}+\sigma_{k_{1}} \pm \sigma_{0} \leq \sigma_{k_{2}}+\sigma_{k_{1}}+\sigma_{0}<\sigma_{t}
$$

contradicting the fact that $t<k_{3}$. The claim follows.

If $k_{1}=1$, it is only possible that $t=k_{2}$, so the second statement of the lemma follows. Suppose now that $k_{1}>1$. We have that $v_{1}=2 e_{0}-e_{1}$ by Corollary 4.10. So if $v_{t}$ is tight with $t>1$, we get that $\left\langle v_{1}, v_{t}\right\rangle=3$ and $\left|v_{t}\right|>\left|v_{1}\right|=5$. Also, since either $t<k_{1}$ or $t=k_{2}$, 
$\left\langle v_{t}, x_{0}\right\rangle=\left\langle v_{1}, x_{0}\right\rangle=2$. Therefore, either $\epsilon_{1}=-1$ and $\left[v_{1}\right]$ has left endpoint 1 , or $\epsilon_{1}=1$ and $\left[v_{1}\right]$ has left endpoint 0 , and the same holds for $\epsilon_{t}$ and $\left[v_{t}\right]$. By Lemma 3.9,

$$
3=\left\langle v_{1}, v_{t}\right\rangle=\epsilon_{1} \epsilon_{t}\left(\left|\left[v_{1} \cap v_{t}\right]\right|-\delta\left(\left[v_{1}\right],\left[v_{t}\right]\right)\right),
$$

$\left|\left[v_{1} \cap v_{t}\right]\right| \geq 2$ and $\delta\left(\left[v_{1}\right],\left[v_{t}\right]\right) \leq 3$, so if $\epsilon_{1} \neq \epsilon_{t}$, the right hand side of this equation is at most 1 . Therefore, $\epsilon_{1}=\epsilon_{t}$, and the left endpoints of $\left[v_{1}\right]$ and $\left[v_{t}\right]$ are equal. Since $\left|v_{t}\right|>\left|v_{1}\right|$, the right endpoint of $\left[v_{t}\right]$ is to the right of the right endpoint of $\left[v_{1}\right]$. This means that $\delta\left(\left[v_{1}\right],\left[v_{t}\right]\right)=1$ and $v_{1} \cap v_{t}=v_{1}$, so

$$
\left\langle v_{1}, v_{t}\right\rangle=\epsilon_{1} \epsilon_{t}\left(\left|\left[v_{1} \cap v_{t}\right]\right|-\delta\left(\left[v_{1}\right],\left[v_{t}\right]\right)\right)=\left|\left[v_{1}\right]\right|-1=4 \neq 3 .
$$

Therefore, $v_{1}$ is the only tight vector.

Lemma 4.12. For $j \neq k_{3},\left\langle v_{j}, x_{0}\right\rangle \geq 0$.

Proof. Using Proposition 4.9, either $x_{0}=e_{0}+e_{k_{1}}+e_{k_{2}}-e_{k_{3}}$ or $x_{0}=e_{0}-e_{k_{1}}-e_{k_{2}}+e_{k_{3}}$. If $x_{0}=e_{0}+e_{k_{1}}+e_{k_{2}}-e_{k_{3}}$, it would only be possible to have $\left\langle v_{j}, x_{0}\right\rangle<0$ for $j=k_{1}$ or $j=k_{2}$. However, in these cases one has $\left\langle v_{j}, x_{0}\right\rangle \geq-1$, and since $\left\langle v_{j}, x_{0}\right\rangle$ is even, it follows that $\left\langle v_{j}, x_{0}\right\rangle \geq 0$. If $x_{0}=e_{0}-e_{k_{1}}-e_{k_{2}}+e_{k_{3}}$, then $\left\langle v_{j}, x_{0}\right\rangle$ is always at least -3 , since $\left\langle v_{j}, e_{0}\right\rangle \geq 0$. Therefore, since it is even, $\left\langle v_{j}, x_{0}\right\rangle \geq-2$. Given that $j \neq k_{3}$, the only possible way to have $\left\langle v_{j}, x_{0}\right\rangle=-2$ is that $k_{1}, k_{2} \in \operatorname{supp}^{+}\left(v_{j}\right)$, and $0, k_{3} \notin \operatorname{supp}^{+}\left(v_{j}\right)$. Observe that this cannot happen since then $v_{j}+x_{0}$ is still of the form $-e_{j}+\sum_{i \in A^{\prime}} e_{i}$ for some $A^{\prime} \subset\{0, \ldots, j-1\}$, but $A^{\prime}$ is lexicographically after $\operatorname{supp}^{+} v_{j}$, contradicting the maximality criterion in Definition 4.1.

Lemma 4.13. If $v_{i}$ and $v_{j}$ are two unbreakable standard basis vectors with $i, j \neq k_{3}$, then it cannot be the case that $\left[v_{i}\right]$ contains $x_{0}$ and $\left[v_{j}\right]$ contains $x_{1}$ but not $x_{0}$. In particular, $\delta\left(\left[v_{i}\right],\left[v_{j}\right]\right) \leq 2$.

Proof. Assume the contrary. Since $i, j \neq k_{3}$, and $k_{3}=\max \operatorname{supp}\left(x_{0}\right)$, neither $v_{i}$ nor $v_{j}$ is equal to $\pm x_{0}$, and by Lemma 4.12, $\left\langle v_{i}, x_{0}\right\rangle$ and $\left\langle v_{j}, x_{0}\right\rangle$ are both nonnegative. Therefore, $\left\langle v_{i}, x_{0}\right\rangle=\left\langle v_{j}, x_{0}\right\rangle=2$. Since $x_{0}$ is contained in $\left[v_{i}\right]$, the left endpoint of $\left[v_{i}\right]$ is 0 and $\epsilon_{i}=1$. Similarly, $\left[v_{j}\right]$ has left endpoint 1 and $\epsilon_{j}=-1$. Therefore, $\delta\left(\left[v_{i}\right],\left[v_{j}\right]\right)$ is either 2 or 3 , and since $v_{i}$ and $v_{j}$ are unbreakable and $a_{1} \geq 3, z_{i}=z_{j}=1$ and $\left|\left[v_{i} \cap v_{j}\right]\right|=\left|v_{i}\right|=\left|v_{j}\right|=a_{1}$. This means that

$$
\left\langle v_{i}, v_{j}\right\rangle=\epsilon_{i} \epsilon_{j}\left(\left|\left[v_{i} \cap v_{j}\right]\right|-\delta\left(\left[v_{i}\right],\left[v_{j}\right]\right)\right)=-\left|v_{i}\right|+\delta\left(\left[v_{i}\right],\left[v_{j}\right]\right)=-\left|v_{j}\right|+\delta\left(\left[v_{i}\right],\left[v_{j}\right]\right)
$$

Since $v_{i}$ and $v_{j}$ are standard basis vectors, $\left\langle v_{i}, v_{j}\right\rangle \geq-1$. Since $\left|v_{i}\right| \geq 3$ and $\delta\left(\left[v_{i}\right],\left[v_{j}\right]\right)$ is either 2 or $3,\left|v_{i}\right|$ is either 3 or 4 . That is, using Equation (7), $\left\langle v_{i}, v_{j}\right\rangle$ is equal to -1 if $\left|v_{i}\right|=4$ and either 0 or -1 if $\left|v_{i}\right|=3$. In particular,

$$
\left\langle v_{i}, v_{j}\right\rangle \leq 0
$$

Using Proposition 4.9, suppose first that $x_{0}=e_{0}+e_{k_{1}}+e_{k_{2}}-e_{k_{3}}$. Then since $\left\langle v_{i}, x_{0}\right\rangle=$ $\left\langle v_{j}, x_{0}\right\rangle=2$ and $i, j \neq k_{3}$, each of $\operatorname{supp}^{+}\left(v_{i}\right)$ and $\operatorname{supp}^{+}\left(v_{j}\right)$ contain at least two of $0, k_{1}$, and $k_{2}$, and $i, j \notin\left\{k_{1}, k_{2}\right\}$. In particular, $\operatorname{supp}^{+}\left(v_{i}\right)$ and $\operatorname{supp}^{+}\left(v_{j}\right)$ intersect, so $\left\langle v_{i}, v_{j}\right\rangle \geq 0$. Therefore, using Equation (7) and the earlier discussion, we must have $\left|v_{i}\right|=\left|v_{j}\right|=3$, so $\operatorname{supp}^{+}\left(v_{i}\right)$ and $\operatorname{supp}^{+}\left(v_{j}\right)$ in fact contain no elements outside of $\left\{0, k_{1}, k_{2}\right\}$. In particular, 
$\operatorname{supp}^{+}\left(v_{i}\right)$ does not contain $j$, and vice versa, $\operatorname{supp}^{+}\left(v_{j}\right)$ does not contain $i$. Therefore, we get that $\left\langle v_{i}, v_{j}\right\rangle \geq 1$ which is a contradiction to (8).

If now $x_{0}=e_{0}-e_{k_{1}}-e_{k_{2}}+e_{k_{3}}$, then since $\left\langle v_{i}, x_{0}\right\rangle=2$ and $i \neq k_{3}$, there are two cases: Case 1 is that $\operatorname{supp}^{+}\left(v_{i}\right)$ contains 0 and $k_{3}$ but not $k_{1}$ and $k_{2}$, and Case 2 is that $i=k_{2}$ or $k_{1}$, $\operatorname{supp}^{+}\left(v_{i}\right)$ contains 0 , and (if $\left.i=k_{2}\right), \operatorname{supp}^{+}\left(v_{i}\right)$ does not contain $k_{1}$. The same holds for $v_{j}$. If one of $v_{i}$ and $v_{j}$ is in Case 1 , then $\left\langle v_{i}, v_{j}\right\rangle \geq 1$, a contradiction to (8). If both $v_{i}$ and $v_{j}$ are in Case 2, we may assume $i=k_{1}$ and $j=k_{2}$, and we still have $\left\langle v_{i}, v_{j}\right\rangle \geq 1$, a contradiction.

Corollary 4.14. If $v_{i}$ and $v_{j}$ are two unbreakable standard basis vectors with $i \neq j$ and $i, j \neq k_{3}$, then $\left|\left\langle v_{i}, v_{j}\right\rangle\right| \leq 1$, with equality if only if $\left[v_{i}\right]$ abuts $\left[v_{j}\right]$.

Proof. If neither $\left[v_{i}\right]$ nor $\left[v_{j}\right]$ contains $x_{0}$, then both $v_{i}$ and $v_{j}$ are contained in a linear sublattice of $C(p, q)$ and this reduces to [Gre13, Lemma 4.4]. Similarly, if one of $\left[v_{i}\right]$ or $\left[v_{j}\right]$ contains $x_{0}$ and the other contains neither $x_{0}$ nor $x_{1}$, or if both $\left[v_{i}\right]$ and $\left[v_{j}\right]$ contain $x_{0}$, then reflecting both $v_{i}$ and $v_{j}$ about $x_{0}^{\perp}$ puts both of them in a linear sublattice of $C(p, q)$. Using Lemma 4.13, these are the only possibilities.

Corollary 4.15. If $v_{i}$ and $v_{j}$ are unbreakable with $\left|v_{i}\right|,\left|v_{j}\right| \geq 3, i \neq j$ and $i, j \neq k_{3}$, then $z_{i} \neq z_{j}$, where $z_{i}$ and $z_{j}$ are defined in Definition 3.15 .

Proof. Suppose for contradiction $x_{z_{i}}=x_{z_{j}}$. By Lemma 4.13, $\delta\left(\left[v_{i}\right],\left[v_{j}\right]\right) \leq 2$. Therefore, using Lemmas 3.9 and 3.10,

$$
\left\langle\left[v_{i}\right],\left[v_{j}\right]\right\rangle=\left|\left[v_{i} \cap v_{j}\right]\right|-\delta\left(\left[v_{i}\right],\left[v_{j}\right]\right)=\left|x_{z_{i}}\right|-\delta\left(\left[v_{i}\right],\left[v_{j}\right]\right) \geq 3-2=1,
$$

By Corollary 4.14, $\left\langle\left[v_{i}\right],\left[v_{j}\right]\right\rangle=1$ and $\left[v_{i}\right]$ abuts $\left[v_{j}\right]$. We would then have $\delta=1$, so the equality in (9) cannot be attained, a contradiction.

Corollary 4.16. There is at most one $j \neq k_{3}$ for which $v_{j}$ is unbreakable and $\left\langle v_{j}, x_{0}\right\rangle$ is nonzero.

Proof. Since $a_{1} \geq 3$, if there exists an unbreakable standard basis element $v_{j}$ for which $\left\langle v_{j}, x_{0}\right\rangle \neq 0, j \neq k_{3}$, then $x_{z_{j}}=x_{1}$. It follows from Corollary 4.15 that there exists at most one such $j$.

Since the pairings of $v_{k_{3}}$ with other standard basis vectors are difficult to control, and since Corollary 4.16 gives good control on the pairings between $x_{0}$ and the other standard basis vectors, it will be easier in what follows if we replace $S$ with the modified basis

$$
S^{\prime}=\left(S \backslash\left\{v_{k_{3}}\right\}\right) \cup\left\{x_{0}\right\} .
$$

The set $S^{\prime}$ is still a basis of $(\sigma)^{\perp}$ because $\left\langle x_{0}, e_{k_{3}}\right\rangle= \pm 1$ but $\left\langle x_{0}, e_{j}\right\rangle=0$ for $j>k_{3}$, so if we write $x_{0}$ as a linear combination of elements of $S$, the coefficient of $v_{k_{3}}$ will be \pm 1 .

Using Lemmas 4.14 and 4.16, we can relate the pairings between elements of $S^{\prime}$ very closely to the geometry of the intervals. It will be convenient to use two graphs associated to a C-type lattice. Recall that the pairing graph $\hat{G}(V)$ for a subset $V$ of a lattice $L$ has vertex set $V$ and an edge $\left(v_{i}, v_{j}\right)$ whenever $\left\langle v_{i}, v_{j}\right\rangle \neq 0$ (Definition 3.3). 
Definition 4.17. If $T$ is a set of irreducible vectors in a C-type lattice $C(p, q)$, the intersection graph $G(T)$ has vertex set $T$, and an edge between $v$ and $w$ if the intervals corresponding to $v$ and $w$ abut. We write $v \sim w$ if $v$ and $w$ are connected in $G(T)$.

Lemma 4.18. If the intervals corresponding to $v$ and $w$ abut, then $\langle v, w\rangle \neq 0$.

Proof. If one of $v, w$ is $x_{0},\langle v, w\rangle= \pm 2 \neq 0$. If none of $v, w$ is $x_{0}$, then $\delta([v],[w])=1$, our conclusion follows from Lemma 3.9.

The following is immediate from Corollary 4.14 and Lemma 4.18:

Proposition 4.19. For $T \subset S^{\prime}, G(T)$ is obtained from $\hat{G}(T)$ by removing some edges incident to breakable vectors.

In particular, if we write $\bar{S}^{\prime}$ for the set of unbreakable elements of $S^{\prime}, G\left(\bar{S}^{\prime}\right)=\hat{G}\left(\bar{S}^{\prime}\right)$. The main use we have for this result is the following structural facts about the intersection graph.

Definition 4.20. A claw in a graph $G$ is a quadruple $\left(v, w_{1}, w_{2}, w_{3}\right)$ of vertices such that $v$ neighbors all the $w_{i}$, but no two of the $w_{i}$ neighbor each other.

Lemma 4.21 (Lemma 4.8 of [Gre13]). The intersection graph $G(T)$ has no claws.

Definition 4.22. Given a set $T$ of unbreakable elements in a C-type lattice and $v_{1}, v_{2}, v_{3} \in T$, $\left(v_{1}, v_{2}, v_{3}\right)$ is a heavy triple if $\left|v_{i}\right| \geq 3$ and $v_{i} \neq \pm x_{0}$ for each $i$, and if each pair among the $v_{i}$ is connected by a path in $G(T)$ disjoint from the third.

Lemma 4.23 (Based on Lemma 4.10 of [Gre13]). $G\left(\bar{S}^{\prime}\right)$ has no heavy triples.

Proof. If $v_{i}, v_{j}$, and $v_{k}$ are unbreakable and have norm at least 3 , and none of them is $\pm x_{0}$, then by Corollary 4.15 we might as well assume $z_{i}<z_{j}<z_{k}$. Then any path from $v_{i}$ to $v_{k}$ in $G\left(\bar{S}^{\prime}\right)$ includes some $v_{\ell} \in \bar{S}^{\prime}$ such that $\left[v_{\ell}\right]$ contains $x_{z_{j}}$, where $\bar{S}^{\prime}$ is defined in (10). But then $\ell=j$, so $\left(v_{i}, v_{j}, v_{k}\right)$ is not heavy.

The proof of the following lemma is identical to [Gre13, Lemma 3.8].

Lemma 4.24. If the elements of $T$ are linearly independent, any cycle in $G(T)$ induces a complete subgraph.

Corollary 4.25 (Based on Lemma 4.11 of [Gre13]). Any cycle in $G\left(\bar{S}^{\prime}\right)$ has length three.

Proof. By Corollary 4.16, any cycle in $G\left(\bar{S}^{\prime}\right)$ does not contain $x_{0}$. Using Lemma 4.24, the cycle will contain at most two vertices of norm $>2$ to avoid producing a heavy triple. (See Definition 3.6.) If it had two vertices of norm 2, using Lemma 4.24, they would have nonzero inner product, so must be of the form $v_{i}=e_{i-1}-e_{i}$ and $v_{i+1}=e_{i}-e_{i+1}$ for some $i$. But for any other $j(j \neq i, i+1)$, Lemma 4.3 implies that $\operatorname{supp}\left(v_{j}\right) \cap\{i-1, i, i+1\}$ is one of $\emptyset$, $\{i+1\},\{i, i+1\}$, or $\{i-1, i, i+1\}$. In none of these cases does $v_{j}$ have nonzero inner product with both $v_{i}$ and $v_{i+1}$, a criterion that must be fulfilled by Lemma 4.24. That is, any cycle in $G\left(\bar{S}^{\prime}\right)$ must be of length three. 
Lemma 4.26. Let $m<N$ be two possitive integers satisfying $k_{3} \notin[m, N]$. Suppose that $v_{m}$ is unbreakable and it neighbors either $x_{0}$ or some unbreakable $v_{j}$ with $j<m$. Suppose that for any index $i$ satisfying $m<i \leq N$, we have $\min \operatorname{supp}\left(v_{i}\right) \geq m$, and $v_{i}$ is unbreakable. Then $\left|v_{i}\right|=2$ for any $i$ satisfying $m<i \leq N$.

Proof. When $i=m+1$, we clearly have $\left|v_{i}\right|=2$. Now assume $\left|v_{i}\right|=2$ for any $i$ satisfying $m<i<l \leq N$, we want to prove $\left|v_{l}\right|=2$. Let $t=\min \operatorname{supp}\left(v_{l}\right) \geq m$, then $v_{l}$ is just right by Lemmas 4.3 and 4.7. If $m<t<l-1$, we would have a claw $\left(v_{t}, v_{l}, v_{t-1}, v_{t+1}\right)$. If $t=m$ and $v_{m}$ neighbors $x_{0}$, we would have a claw $\left(v_{m}, v_{l}, x_{0}, v_{m+1}\right)$ by Corollary 4.16. If $t=m$ and $v_{m}$ neighbors an unbreakable $v_{j}$ with $j<m$, we would have a claw $\left(v_{m}, v_{l}, v_{j}, v_{m+1}\right)$. So $t=l-1$ and $\left|v_{l}\right|=2$.

\section{5. $k_{1}=1, k_{2}>2$}

In this section we consider, in the notation of Proposition 4.9, the case where $k_{1}=1$ and $k_{2}>2$. Using Corollary 4.10, one has

$$
x_{0}=e_{0}+e_{1}+e_{k_{2}}-e_{k_{3}},
$$

where $k_{2}>2$. Also, we have that $v_{1}=e_{0}-e_{1}$. So

$$
\sigma_{0}=\sigma_{1}=1 \text {. }
$$

By Lemmas 4.5 and 4.11, the only possible breakable vector is $v_{k_{2}}$. In what follows we classify all changemaker vectors whose orthogonal complements are isomorphic to C-type lattices with $x_{0}$ as given in (11) and $k_{2}>2$. We start by determining the first $k_{3}+1$ components of such changemaker vectors.

Proposition 5.1. If $k_{1}=1$ and $k_{2}>2$, the initial segment $\left(\sigma_{0}, \sigma_{1}, \cdots, \sigma_{k_{3}}\right)$ of $\sigma$ is equal to $\left(1,1,2^{[s]}, \sigma_{k_{2}}, \sigma_{k_{2}}+2\right)$ for some $s>0$.

Proof. We start by observing that, using Lemma 3.5, we must have $v_{2}=e_{0}+e_{1}-e_{2}$. So $\sigma_{2}=2$. By Corollary 4.16, $\min \operatorname{supp}\left(v_{i}\right) \geq 2$ for all $2<i<k_{2}$. It follows from Lemma 4.26 that $\left|v_{i}\right|=2$ for all $2<i<k_{2}$. So $\sigma_{i}=2$ for $2 \leq i<k_{2}$. Now, using (11) and (12) together with the fact that $\left\langle\sigma, x_{0}\right\rangle=0$, we get that $\sigma_{k_{3}}=\sigma_{k_{2}}+2$. We claim that $k_{3}=k_{2}+1$. Suppose for contradiction that $k_{3} \neq k_{2}+1$. The component $\sigma_{k_{2}+1}$ must be between $\sigma_{k_{2}}$ and $\sigma_{k_{2}}+2=\sigma_{k_{3}}$. If $\sigma_{k_{2}+1}$ is equal to either $\sigma_{k_{2}}$ or $\sigma_{k_{3}}$, there will be an element $v \in(\sigma)^{\perp}$ with $\left\langle v, x_{0}\right\rangle=1$, contradicting Lemma 3.5. If $\sigma_{k_{2}+1}=\sigma_{k_{2}}+1$, then $v_{k_{2}+1}=e_{1}+e_{k_{2}}-e_{k_{2}+1}$. But then $\left\langle v_{k_{2}+1}, x_{0}\right\rangle=2 \neq 0$, contradicting Corollary 4.16 since $\left\langle v_{2}, x_{0}\right\rangle=2$. This finishes the proof.

Corollary 5.2. In the situation of Proposition 5.1, the component $\sigma_{k_{2}}$ of the changemaker vector is one of $2 s-1,2 s+1$, or $2 s+3$. These correspond to $v_{k_{2}}$ being gappy, just right, or tight, respectively.

Proof. If $v_{k_{2}}$ is tight, the third of these possibilities occurs. If not, using Corollary 4.16, we get that $\left\langle v_{k_{2}}, x_{0}\right\rangle=0$. (Note that $\left\langle v_{2}, x_{0}\right\rangle=2$.) So $1 \in \operatorname{supp}^{+}\left(v_{k_{2}}\right)$ and $0 \notin \operatorname{supp}^{+}\left(v_{k_{2}}\right)$. Since 

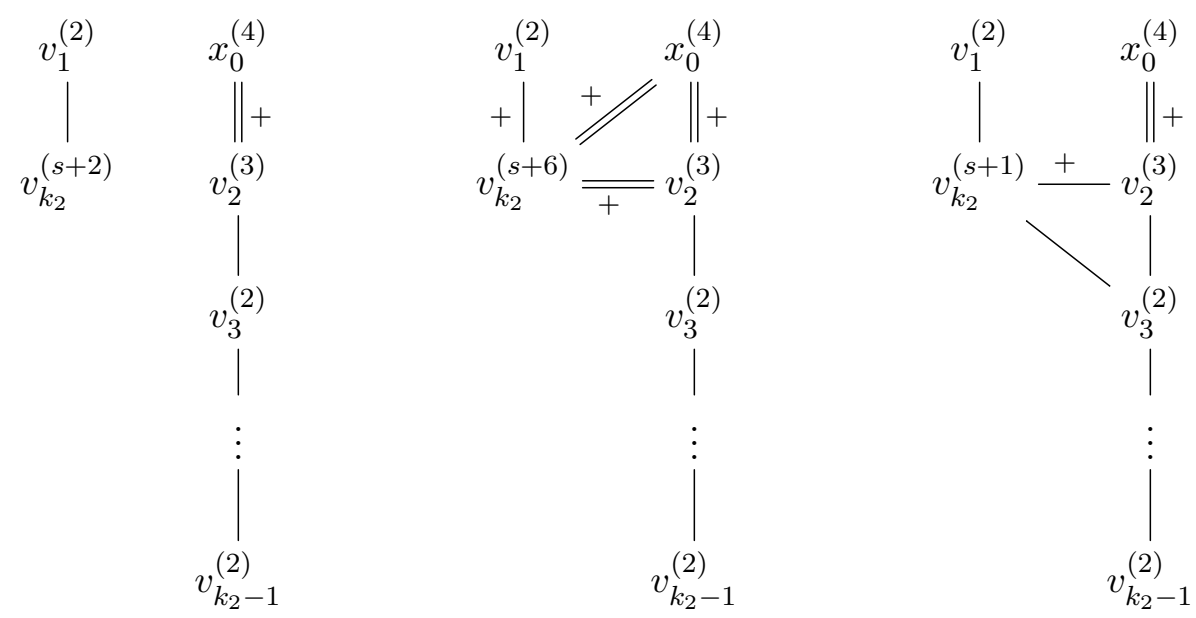

FiguRE 7. Pairing graphs of the standard basis when $v_{k_{2}}$ is just right (left), tight (center), and gappy (right). Superscripts give the norm of the basis vector, the number of edges gives the absolute value of the inner product, and an edge is labelled with + if the inner product is positive.

$\left|v_{j}\right|=2$ for $2<j<k_{2}$, Lemma 4.3 implies that the only possible gappy index for $v_{k_{2}}$ is 1 , so

$$
v_{k_{2}}=e_{1}+e_{j}+e_{j+1}+\cdots+e_{k_{2}-1}-e_{k_{2}},
$$

for some $1<j<k_{2}$. If $j>3$, the pairing graph will have a cycle on $v_{2}, \cdots, v_{j}, v_{k_{2}}$ of length larger than 3, contradicting Corollary 4.25. In particular, if 1 is indeed a gappy index for $v_{k_{2}}$, then $j=3$, and $\sigma_{k_{2}}=2 s-1$. Otherwise one has $j=2$, and therefore $\sigma_{k_{2}}=2 s+1$.

It turns out that the classification will highly depend on the type of the vector $v_{k_{2}}$ : whether it is tight, just right, or gappy. For $j>k_{3}$, let

$$
S_{j}=\operatorname{supp}\left(v_{j}\right) \cap\left\{0,1, \ldots, k_{3}\right\},
$$

and let

$$
S_{j}^{\prime}=\operatorname{supp}\left(v_{j}\right) \cap\left\{0,1, k_{2}, k_{3}\right\} .
$$

Given that $\left\langle v_{2}, x_{0}\right\rangle=2$ and, using Corollary 4.16, we must have $\left\langle v_{j}, x_{0}\right\rangle=0$, and that $S_{j}^{\prime}$ is one of $\emptyset,\left\{1, k_{3}\right\}$, or $\left\{k_{2}, k_{3}\right\}$ by Lemma 4.3. Figure 7 depicts the paring graphs of the possible changemaker C-type lattices on their first $k_{3}$ vectors of the basis $S^{\prime}$, defined in (10), depending on the type of $v_{k_{2}}$. With a slight abuse of notation, we often use $v_{k_{3}}$ in place of $x_{0}$.

With the notation of this section in place:

Lemma 5.3. If $S_{j}^{\prime}=\emptyset, S_{j}$ is either $\emptyset$ or $\left\{k_{2}-1\right\}$. In the second case, $v_{k_{2}}$ is not gappy.

Proof. Set $i=\min S_{j}$. Suppose for contradiction that $S_{j}$ is nonempty and $i<k_{2}-1$. If $i>2$, then there will be a claw on $v_{i}, v_{i+1}, v_{i-1}, v_{j}$. If $i=2$ there will be a claw $\left(v_{2}, v_{3}, x_{0}, v_{j}\right)$. Therefore, $i=k_{2}-1$, and so the first statement follows. If $S_{j}=\left\{k_{2}-1\right\}$, then $\left\langle v_{j}, v_{k_{2}-1}\right\rangle=-1$, 
$\left\langle v_{j}, v_{k_{2}}\right\rangle=1$, and $\left\langle v_{j}, v_{i}\right\rangle=0$ for all other $i \leq k_{3}$, so if $v_{k_{2}}$ is gappy there is a claw $\left(v_{k_{2}}, v_{1}, v_{2}, v_{j}\right)$ (see Figure 7).

Lemma 5.4. If $S_{j}^{\prime}=\left\{k_{2}, k_{3}\right\}, S_{j}$ is either $\left\{k_{2}, k_{3}\right\}$ or $\left\{k_{2}-1, k_{2}, k_{3}\right\}$. In either case, $v_{k_{2}}$ is not gappy.

Proof. Again, set $i=\min S_{j}$. If $i<k_{2}-1$, there will be a claw on either $v_{i}, v_{i+1}, v_{i-1}, v_{j}$ or $v_{2}, v_{3}, x_{0}, v_{j}$, depending on whether $i>2$ or $i=2$. So the first statement follows. Corresponding to the two possibilities for $S_{j}$, the vector $v_{j}$ will have nonzero inner product with either $v_{k_{2}}$ or $v_{k_{2}-1}$, but no other $v_{i}$ with $i \leq k_{3}$. If $v_{k_{2}}$ is gappy, this creates a claw $\left(v_{k_{2}}, v_{1}, v_{2}, v_{j}\right)$ in the first case, and a heavy triple $\left(v_{2}, v_{k_{2}}, v_{j}\right)$ in the second: again, see Figure 7.

Lemma 5.5. If $S_{j}^{\prime}=\left\{1, k_{3}\right\}$, either $S_{j}$ is one of $\left\{1,2,3, \ldots, k_{2}-1, k_{3}\right\}$ and $\left\{1,3, \ldots, k_{2}-1, k_{3}\right\}$ and $v_{k_{2}}$ is tight, or $S_{j}=\left\{1, k_{3}\right\}, s=1$, and $v_{k_{2}}$ is not gappy.

Proof. Using Lemma 4.3 , none of $2, \ldots, k_{2}-2$ can be a gappy index for $v_{j}$. Thus, we must have either $S_{j}=\left\{1, k_{3}\right\}$ or $S_{j}=\left\{1, k, k+1, \ldots, k_{2}-1, k_{3}\right\}$ for some $1<k<k_{2}$.

In the first case, $v_{j}$ will have nonzero inner product with just $v_{1}, v_{2}$, and $v_{k_{2}}$. If $v_{k_{2}}$ is gappy, this creates a heavy triple $\left(v_{2}, v_{k_{2}}, v_{j}\right)$. If $v_{k_{2}}$ is just right or tight, this creates a claw $\left(v_{2}, v_{j}, x_{0}, v_{3}\right)$, unless $s=1$ : see Figure 7 .

In the second case, to avoid a cycle $\left(v_{2}, v_{3}, \ldots, v_{k}, v_{j}\right)$ of length longer than 3 (Corollary 4.25) we must have $k$ equal to 2 or 3 . Then $\left\langle v_{j}, v_{k_{2}}\right\rangle$ is either $s$ or $s+1$, and unless $v_{k_{2}}$ is tight this must be at most 1 (Corollary 4.14). Since $s \geq 1$, if $v_{k_{2}}$ is not tight, we must have $\left\langle v_{j}, v_{k_{2}}\right\rangle=s=1$. Note that in this case $k_{3}=4, k_{2}=3, v_{k_{2}}=e_{1}+e_{2}-e_{3}$, and $S_{j}=\{1,2,4\}$. Consequently, $\left\langle v_{j}, v_{3}\right\rangle=2$, again contradicting Corollary 4.14.

Proposition 5.6. If $v_{k_{2}}$ is gappy, then $s \geq 2$ and $n+1=k_{3}$ (i.e. $v_{k_{3}}$ is the last standard basis vector). The corresponding changemaker vectors are

$$
\left(1,1,2^{[s]}, 2 s-1,2 s+1\right), s \geq 2 .
$$

Proof. By Corollary 5.2, $\sigma_{k_{2}}=2 s-1 \geq 2$, so $s \geq 2$. By Lemmas 5.5, 5.4, and 5.3, we get that $S_{j}=\emptyset$ for all $j>k_{3}$. If $v_{k_{3}+1}$ existed it would have $k_{3} \in S_{k_{3}+1}$.

Proposition 5.7. If $v_{k_{2}}$ is just right, then one of the following holds:

(1) $v_{k_{3}+1}=e_{k_{2}}+e_{k_{3}}-e_{k_{3}+1}, v_{k_{3}+2}=e_{k_{2}-1}+e_{k_{2}}+e_{k_{3}}+e_{k_{3}+1}-e_{k_{3}+2}$, and $k_{3}+2=n+1$.

(2) $v_{k_{3}+1}=e_{k_{2}-1}+e_{k_{2}}+e_{k_{3}}-e_{k_{3}+1}, v_{k_{3}+2}=e_{k_{2}}+e_{k_{3}}+e_{k_{3}+1}-e_{k_{3}+2}$, and $k_{3}+2=n+1$.

(3) $s=1$, so $k_{2}=3$. $v_{5}=e_{3}+e_{4}-e_{5},\left|v_{i}\right|=2$ for $5<i<\ell, v_{\ell}=e_{1}+e_{4}+e_{5}+\cdots+e_{\ell-1}-e_{\ell}$, and either $v_{\ell+1}=e_{\ell-1}+e_{\ell}-e_{\ell+1}$ and $\left|v_{i}\right|=2$ for $i>\ell+1$, or $\ell=n+1$.

(4) $s=1$, so $k_{2}=3$. $v_{5}=e_{1}+e_{4}-e_{5}$, and either $v_{6}=e_{3}+e_{4}+e_{5}-e_{6}$ and $\left|v_{i}\right|=2$ for $i>6$ or $5=n+1$.

The corresponding changemaker vectors are

(1) $\left(1,1,2^{[s]}, 2 s+1,2 s+3,4 s+4,8 s+10\right), s \geq 1$.

(2) $\left(1,1,2^{[s]}, 2 s+1,2 s+3,4 s+6,8 s+10\right), s \geq 1$. 
(3) $\left(1,1,2,3,5,8^{[s]}, 8 s+6,8 s+14^{[t]}\right), s, t \geq 0$, (the parameter $s$ in this family is not the previous s.)

Proof. We divide the proof into two cases, based on whether or not there is some $\ell$ with $S_{\ell}=\left\{1, k_{3}\right\}$. If there is no such $\ell$, then by Lemmas 5.5, 5.4, and 5.3, for any $j>k_{3}$, $S_{j}$ is either empty or one of the three possibilities: $\left\{k_{2}-1\right\},\left\{k_{2}, k_{3}\right\}$, or $\left\{k_{2}-1, k_{2}, k_{3}\right\}$. If $S_{j}=\left\{k_{2}-1\right\},\left\langle v_{j}, v_{k_{2}-1}\right\rangle$ and $\left\langle v_{j}, v_{k_{2}}\right\rangle$ are both nonzero, but $\left\langle v_{j}, v_{i}\right\rangle=0$ for all other $i \leq k_{3}$. If $S_{j}=\left\{k_{2}, k_{3}\right\},\left\langle v_{j}, v_{k_{2}}\right\rangle$ is nonzero but $\left\langle v_{j}, v_{i}\right\rangle=0$ for all other $i \leq k_{3}$, and if $S_{j}=\left\{k_{2}-1, k_{2}, k_{3}\right\}$ only $\left\langle v_{j}, v_{k_{2}-1}\right\rangle$ is nonzero. In particular, no $v_{j}$ with $j \leq k_{3}$ except for $v_{k_{2}}$ and $v_{k_{2}-1}$ can have nonzero pairing with $v_{i}$ for some $i>k_{3}$. Furthermore, for $j$ equal to either $k_{2}$ or $k_{2}-1$, we claim that there can be at most one $i>k_{3}$ with $\left\langle v_{j}, v_{i}\right\rangle$ nonzero: if there were two, there would be either a claw if they did not neighbor each other, or a heavy triple if they did. See Figure 7. (For instance, if $v_{r}$ and $v_{t}$, with $r, t>k_{3}$, both have nonzero pairing with $v_{k_{2}-1}$, and also if $v_{r}$ and $v_{t}$ pair with each other, then there will be a heavy triple $\left(v_{r}, v_{t}, v_{2}\right)$.) Since the pairing graph of a basis must be connected, there in fact must be some $j>k_{3}$ with $\left\langle v_{j}, v_{k_{2}}\right\rangle$ nonzero, and some $j>k_{3}$ with $\left\langle v_{j}, v_{k_{2}-1}\right\rangle$ nonzero. This has two implications. First that the vector $v_{k_{3}+1}$ exists, and either $S_{k_{3}+1}=\left\{k_{2}, k_{3}\right\}$ or $S_{k_{3}+1}=\left\{k_{2}-1, k_{2}, k_{3}\right\}$. Second, there is another index $j^{\prime}>k_{3}+1$ with $S_{j^{\prime}}$ equal to the other of these two possibilities of $S_{k_{3}+1}$.

It remains only to show that $j^{\prime}=k_{3}+2$, and that there is no further standard basis vector. Since $S_{k_{3}+1} \cap S_{j^{\prime}}=\left\{k_{2}, k_{3}\right\}$, in order to keep $\left\langle v_{k_{3}+1}, v_{j^{\prime}}\right\rangle \leq 1$ (Corollary 4.14), it must be the case that $k_{3}+1 \in \operatorname{supp}^{+}\left(v_{j^{\prime}}\right)$, and in this case $\left\langle v_{k_{3}+1}, v_{j^{\prime}}\right\rangle=1$. Therefore, $v_{k_{3}+1}$ and $v_{j^{\prime}}$ are adjacent in the intersection graph. If $j^{\prime}>k_{3}+2$, then since $S_{k_{3}+2}=\emptyset$, we get that $\left|v_{k_{3}+2}\right|=2$. Therefore, using Lemma $4.3, k_{3}+1$ cannot be a gappy index for $v_{j^{\prime}}$, so $k_{3}+2 \in$ $\operatorname{supp}^{+}\left(v_{j^{\prime}}\right)$. This means that $\left\langle v_{j^{\prime}}, v_{k_{3}+2}\right\rangle=0$, so there is a claw on either $v_{k_{3}+1}, v_{k_{2}}, v_{k_{3}+2}, v_{j^{\prime}}$ or $v_{k_{3}+1}, v_{k_{2}-1}, v_{k_{3}+2}, v_{j^{\prime}}$, depending on the possibilities for $S_{k_{3}+1}$. Therefore, $j^{\prime}=k_{3}+2$.

Finally, if $v_{k_{3}+3}$ existed, it would have $S_{k_{3}+3}=\emptyset$, so would equal either $e_{k_{3}+1}+e_{k_{3}+2}-e_{k_{3}+3}$ or $e_{k_{3}+2}-e_{k_{3}+3}$. Therefore, $v_{k_{3}+3}$ would have nonzero inner product with either $v_{k_{3}+1}$ or $v_{k_{3}+2}$ but not both, hence we get a claw centered at either $v_{k_{3}+1}$ or $v_{k_{3}+2}$.

If there is some $\ell$ with $S_{\ell}=\left\{1, k_{3}\right\}$, then $s=1$ by Lemma 5.5. In this case, $\left\langle v_{\ell}, v_{1}\right\rangle=-1$, $\left\langle v_{\ell}, v_{2}\right\rangle=1, k_{2}=3$, and $\left\langle v_{\ell}, v_{k_{2}}\right\rangle=1$. If, for any $i>k_{3}$ with $i \neq \ell$, we had $\left\langle v_{i}, v_{2}\right\rangle \neq 0$, there would be either a claw $\left(v_{2}, x_{0}, v_{i}, v_{\ell}\right)$ or a heavy triple $\left(v_{2}, v_{i}, v_{\ell}\right)$ depending on whether or not $\left[v_{i}\right]$ and $\left[v_{\ell}\right]$ abut. Since we must have $\left\langle v_{i}, v_{2}\right\rangle=0$ for all $i>k_{3}$ with $i \neq \ell$, the set $S_{i}$ cannot be $\left\{1, k_{3}\right\},\left\{k_{2}-1\right\}$ or $\left\{k_{2}-1, k_{2}, k_{3}\right\}$, so by Lemmas 5.35 .4 and 5.5 ,

$$
S_{i}=\emptyset \text { or }\left\{k_{2}, k_{3}\right\} \text {. }
$$

Also, we have

$$
\left\langle v_{i}, v_{\ell}\right\rangle=0, \quad \text { for any } i>k_{3} \text { with } i \neq \ell \text {. }
$$

Otherwise, either $S_{i}=\emptyset$ in which case there would be a claw $\left(v_{\ell}, v_{1}, v_{2}, v_{i}\right)$, or $S_{i}=\left\{k_{2}, k_{3}\right\}$ and there would be a heavy triple $\left(v_{i}, v_{\ell}, v_{k_{2}}\right)$.

Now, $k_{3} \in S_{k_{3}+1}$ (Lemma 4.7), so $S_{k_{3}+1}^{\prime}$ is either $\left\{1, k_{3}\right\}$ or $\left\{k_{2}, k_{3}\right\}$. It follows from Lemmas 5.4 and 5.5 and (15) that $S_{k_{3}+1}=S_{k_{3}+1}^{\prime}$. If $S_{k_{3}+1}=\left\{1, k_{3}\right\}$, from (16) we get that $\left\langle v_{k_{3}+2}, v_{k_{3}+1}\right\rangle=$ 0 if $n+1 \geq k_{3}+2$, and therefore by (15), $S_{k_{3}+2}=\left\{k_{2}, k_{3}\right\}$. We claim that $S_{i}=\emptyset$ for $i>k_{3}+2$, 
and also $k_{3}+1 \notin \operatorname{supp}\left(v_{i}\right)$. Note that from (15) if $S_{i} \neq \emptyset$, one necessarily has $S_{i}=\left\{k_{2}, k_{3}\right\}$. Also, to avoid pairing with $v_{k_{3}+1}$, it must be the case that $k_{3}+1 \in \operatorname{supp}^{+}\left(v_{i}\right)$, but this would imply $\operatorname{supp}^{+}\left(v_{i}\right) \cap \operatorname{supp}^{+}\left(v_{k_{3}+2}\right)=\left\{k_{2}, k_{3}, k_{3}+1\right\}$ hence $\left\langle v_{i}, v_{k_{3}+2}\right\rangle \geq 2$, contradicting Corollary 4.14. So $S_{i}=\emptyset$, hence $k_{3}+1 \notin \operatorname{supp}\left(v_{i}\right)$ by (16). This justifies the claim. It follows from Lemma 4.26 that $\left|v_{i}\right|=2$ for $i>k_{3}+2$. This is the last of the possibilities listed in the statement of the proposition.

Lastly, suppose that $S_{k_{3}+1}=\left\{k_{2}, k_{3}\right\}$ (note that $S_{\ell}=\left\{1, k_{3}\right\}$ ). When $i>k_{3}+1$ and $i \neq \ell, S_{i} \neq\left\{k_{2}, k_{3}\right\}$, otherwise we get a heavy triple $\left(v_{i}, v_{k_{2}}, v_{k_{3}+1}\right)$. So $S_{i}=\emptyset$ by (15). By Lemma 4.26, $\left|v_{i}\right|=2$ for $k_{3}+1<i<\ell$. By (16), $v_{\ell}$ is orthogonal to all of $v_{k_{3}+1}, \ldots, v_{\ell-1}$, so all of $k_{3}+1, \ldots, \ell-1$ are members of $\operatorname{supp} v_{\ell}$, forcing $v_{\ell}$ to be of the listed form. If $n+1 \geq l+1$, $v_{\ell+1}$ is also orthogonal to $v_{\ell}$, so $\operatorname{supp} v_{\ell+1} \cap\left\{k_{3}+1, \ldots, \ell-1\right\}$ contains exactly one element, which must be $\ell-1$ by Lemma 4.3. It follows that $v_{\ell+1}=e_{\ell-1}+e_{\ell}-e_{\ell+1}$, as desired. If, for some $i>\ell+1,\left\langle v_{i}, v_{\ell-1}\right\rangle$ is nonzero, then $\ell-1 \in \operatorname{supp}\left(v_{i}\right)$, and $\ell \in \operatorname{supp}\left(v_{i}\right)$ by (16), so $\left\langle v_{i}, v_{\ell+1}\right\rangle \neq 0$ and hence $\left(v_{k_{3}+1}, v_{\ell+1}, v_{i}\right)$ is a heavy triple. Therefore, $v_{i}$ is orthogonal to both $v_{\ell-1}$ and $v_{\ell}$ for $i>\ell+1$, so by Lemma $4.3 \min \operatorname{supp} v_{i} \geq \ell+1$. Then Lemma 4.26 implies that $\left|v_{i}\right|=2$ for $i>\ell+1$, so we are in the third listed situation.

Lemma 5.8. If $v_{k_{2}}$ is tight, $S_{j}$ is one of $\emptyset,\left\{k_{2}-1\right\}$, or $\left\{1,2,3, \ldots, k_{2}-1, k_{3}\right\}$ for each $j>k_{3}$.

Proof. By Lemmas 5.5, 5.4, and 5.3, it suffices to show that $S_{j}$ cannot be $\left\{k_{2}, k_{3}\right\},\left\{k_{2}-\right.$ $\left.1, k_{2}, k_{3}\right\},\left\{1,3, \ldots, k_{2}-1, k_{3}\right\}$, or $\left\{1, k_{3}\right\}$. In the first case, $\left\langle v_{j}, v_{k_{2}}\right\rangle=-1$ and $\left\langle v_{j}, v_{i}\right\rangle=0$ for all other $i \leq k_{3}$. In particular since $v_{j}$ is orthogonal to $v_{1}$ and $v_{2}, v_{j}$ cannot neighbor $v_{k_{2}}$ in the intersection graph without creating a claw. Therefore, $\left[v_{j}\right] \pitchfork\left[v_{k_{2}}\right]$, and so $\delta\left(\left[v_{j}\right],\left[v_{k_{2}}\right]\right)=2$. In order to have $\left\langle v_{j}, v_{k_{2}}\right\rangle=-1$, then, we must have $\left|v_{j}\right|=\left|\left[v_{j} \cap v_{k_{2}}\right]\right|=3$ and $\epsilon_{j}=-\epsilon_{k_{2}}$. Since $\epsilon_{j}=-\epsilon_{k_{2}}$ and $\left[v_{j}\right] \pitchfork\left[v_{k_{2}}\right], v_{j}+v_{k_{2}}$ is the sum of two distant intervals, so is reducible. However, since $\left|v_{j}\right|=3, j=k_{3}+1$ and $v_{j}=e_{k_{2}}+e_{k_{3}}-e_{k_{3}+1}$, and so $v_{k_{2}}+v_{j}$ is irreducible by Lemma 4.6.

In the second case, $\left\langle v_{j}, v_{k_{2}-1}\right\rangle=-1$ and all other $\left\langle v_{j}, v_{i}\right\rangle$ with $i \leq k_{3}$ are zero. Since $\left\langle v_{2}, x_{0}\right\rangle \neq$ $0,\left[v_{2}\right]$ contains $x_{1}$, so $3=\left|v_{2}\right|=\left|x_{1}\right|$. Since $\left|v_{k_{2}}\right|>3$, $\left[v_{k_{2}}\right]$ contains high weight elements other than $x_{1}$. Since $\left[v_{2}\right]$ contains $x_{1}$ and $v_{k_{2}-1}$ is connected by a path of norm-two vectors to $v_{2}$, the unique high weight element $x_{z_{j}}$ of $\left[v_{j}\right]$ is contained in $\left[v_{k_{2}}\right]$. This implies that $\left\langle v_{j}, v_{k_{2}}\right\rangle$ must be nonzero, a contradiction.

In the last two cases, $v_{j}$ has nonzero inner product with both $v_{1}$ and $v_{2}$, so $\left[v_{j}\right]$ abuts both $\left[v_{1}\right]$ and $\left[v_{2}\right]$. Since $\left[v_{1}\right]$ and $\left[v_{2}\right]$ abut $\left[v_{k_{2}}\right]$ at opposite ends, $\left[v_{k_{2}}\right]$ must be contained in the union of $\left[v_{1}\right],\left[v_{2}\right]$, and $\left[v_{j}\right]$. However, $\left\langle v_{j}, v_{k_{2}}\right\rangle \leq s$, so $\left|v_{j}\right| \leq s+\delta\left(\left[v_{k_{2}}\right],\left[v_{j}\right]\right) \leq s+2$. This means that there are only two high weight elements in $\left[v_{k_{2}}\right]$, with one being $x_{1}$ and the other having norm at most $s+2$, so by Lemma 3.10, $\left|v_{k_{2}}\right| \leq s+3$. This contradicts the fact that $\left|v_{k_{2}}\right|=s+6$.

Proposition 5.9. If $v_{k_{2}}$ is tight, $v_{k_{3}+1}=e_{1}+e_{2}+\cdots+e_{k_{2}-1}+e_{k_{3}}-e_{k_{3}+1}, v_{k_{3}+2}$ is either $e_{k_{3}+1}-e_{k_{3}+2}$ or $e_{k_{2}-1}+e_{k_{3}+1}-e_{k_{3}+2}$, and $\left|v_{j}\right|=2$ for all $j>k_{3}+2$. (None of the vectors past $v_{k_{3}}$ are necessary to make the lattice $C$-type $-n+1$ could be $k_{3}$ or anything larger.)

The corresponding changemaker vectors are 
(1) $\left(1,1,2^{[s]}, 2 s+3,2 s+5,4 s+6^{[t]}\right), s \geq 1, t \geq 0$.

(2) $\left(1,1,2^{[s]}, 2 s+3,2 s+5,4 s+6,4 s+8^{[t]}\right), s \geq 1, t \geq 1$.

Proof. Since $k_{3} \in \operatorname{supp}\left(v_{k_{3}+1}\right), S_{k_{3}+1}$ is necessarily equal to $\left\{1,2,3, \ldots, k_{2}-1, k_{3}\right\}$ by Lemma 5.8 , and so $v_{k_{3}+1}=e_{1}+e_{2}+\cdots+e_{k_{2}-1}+e_{k_{3}}-e_{k_{3}+1}$. For any other $j$ with $S_{j}=S_{k_{3}+1}$, we get that $\left\langle v_{j}, v_{k_{3}+1}\right\rangle \geq k_{2}-1 \geq 2$, contradicting Corollary 4.14. Therefore, for $j>k_{3}+1, S_{j}$ is either $\emptyset$ or $\left\{k_{2}-1\right\}$. Suppose for some $j>k_{3}+1$ we have $S_{j}=\left\{k_{2}-1\right\}$. Then $\left\langle v_{j}, v_{k_{2}}\right\rangle=1$ while $v_{j}$ is orthogonal to both $x_{0}$ and $v_{1}$. Since $\left\langle v_{k_{2}}, v_{1}\right\rangle=1$ and $\left\langle x_{0}, v_{1}\right\rangle=0,\left[v_{1}\right]$ abuts the right endpoint of $\left[v_{k_{2}}\right]$. Hence $x_{z_{j}} \in\left[v_{k_{2}}\right]$. By Lemma 3.9, we get that $\left|v_{j}\right|=3$, and $\epsilon_{j}=\epsilon_{k_{2}}$. Since also $\left\langle v_{k_{3}+1}, v_{k_{2}}\right\rangle=s+1, \epsilon_{k_{3}+1}=\epsilon_{k_{2}}=\epsilon_{j}$, so $\left\langle v_{j}, v_{k_{3}+1}\right\rangle$ is either -1 or 0 depending on whether their intervals abut. However, since $\left|v_{j}\right|=3, v_{j}=e_{k_{2}-1}+e_{j-1}-e_{j}$, so $\left\langle v_{j}, v_{k_{3}+1}\right\rangle$ is 1 if $j>k_{3}+2$ and 0 if $j=k_{3}+2$. Therefore, $j=k_{3}+2$ and $S_{i}=\emptyset$ for $i>k_{3}+2$. For any $i>k_{3}+2$, if $\min \operatorname{supp}\left(v_{i}\right)=k_{3}+1, v_{i} \sim v_{k_{3}+1}$. Since $v_{k_{3}+1} \sim v_{1},\left\langle v_{k_{3}+1}, v_{k_{2}}\right\rangle \neq 0$ and $\left[v_{1}\right]$ abuts the right endpoint of $\left[v_{k_{2}}\right], x_{z_{k_{3}+1}}$ is the rightmost high weight vertex in $\left[v_{k_{2}}\right]$ and $\left[v_{1}\right]$ abuts the right endpoint of $\left[v_{k_{3}+1}\right]$. As $\left\langle v_{i}, v_{k_{2}}\right\rangle=0,\left[v_{i}\right]$ must abut the right endpoint of $\left[v_{k_{3}+1}\right]$. We then conclude that $\left[v_{1}\right]$ and $\left[v_{i}\right]$ abut, which is impossible. So min $\operatorname{supp}\left(v_{i}\right)>k_{3}+1$ when $i>k_{3}+2$. Using Lemma 4.26, we conclude that $\left|v_{i}\right|=2$ for $i>k_{3}+2$.

$$
\text { 6. } k_{1}=1, k_{2}=2
$$

In this section we consider the case where $k_{1}=1$ and $k_{2}=2$. Using Corollary 4.10, we get that

$$
x_{0}=e_{0}+e_{1}+e_{2}-e_{k_{3}} .
$$

Also, we have that $v_{1}=e_{0}-e_{1}$. So

$$
\sigma_{0}=\sigma_{1}=1
$$

By Lemma 4.11, the only possible tight vector is $v_{2}$. In what follows we classify all the changemaker vectors whose orthogonal complements are isomorphic to C-type lattices with $x_{0}$ as given in (17). As in the previous section, we start by determining the first $k_{3}+1$ components of such changemaker vectors. It turns out that the initial segment of $\sigma$ depends on whether or not $v_{2}$ is tight.

Lemma 6.1. If $v_{2}$ is tight, the initial segment $\left(\sigma_{0}, \sigma_{1}, \cdots, \sigma_{k_{3}}\right)$ of $\sigma$ is equal to $(1,1,3,5)$.

Proof. By assumption, $v_{2}=2 e_{0}+e_{1}-e_{2}$, so $\sigma_{2}=3$ and $\left|v_{2}\right|=6$. This together with (17) and (18), yields $\sigma_{k_{3}}=5$. We claim that $k_{3}=k_{2}+1=3$. Suppose for contradiction that $k_{3} \neq k_{2}+1$. Recall from Lemma 4.11 that $v_{k_{2}+1}$ cannot be tight. By combining this together with Lemma 3.5, it can only be the case that $\sigma_{k_{2}+1}=4$ and $v_{3}=e_{1}+e_{2}-e_{3}$. Note that $\left\langle v_{2}, x_{0}\right\rangle=2,\left\langle v_{1}, x_{0}\right\rangle=0$, and $\left\langle v_{1}, v_{2}\right\rangle=1$. Therefore, $\left[v_{1}\right]$ abuts the right endpoint of $\left[v_{2}\right]$. Given that $\left[v_{3}\right]$ abuts both $x_{0}$ and $\left[v_{1}\right]$, it follows that the only high weight vertex of $\left[v_{2}\right]$ is that of $\left[v_{3}\right]$ (see Definition 3.6 and Lemma 3.14). This implies that $\left|\left[v_{2}\right]\right|=\left|\left[v_{3}\right]\right|=3$ which is a contradiction. Hence $k_{3}=3$ and $v_{3}=e_{0}+e_{1}+e_{2}-e_{3}$.

Lemma 6.2. If $v_{2}$ is not tight, the initial segment $\left(\sigma_{0}, \sigma_{1}, \cdots, \sigma_{k_{3}}\right)$ of $\sigma$ is equal to either $(1,1,1,3)$ or $(1,1,1,2,3)$. 
Proof. When $v_{2}$ is not tight, using Lemma 3.5 together with the fact that $k_{2}=2$, we get that $v_{2}=e_{1}-e_{2}$, so $\sigma_{2}=1$. This together with (17) and (18), gives us that $\sigma_{k_{3}}=3$. Either $k_{3}=3$ and we get the first possibility stated in the proposition, or $k_{3}>3$. In the latter case, using Lemmas 3.5 and 4.7 , we must have that $v_{3}=e_{1}+e_{2}-e_{3}$, so $\sigma_{3}=2$. We claim that, if $k_{3}>3$, then $k_{3}=4$. If $k_{3} \neq 4$, then we must have $v_{4}=e_{3}-e_{4}$. That will produce a claw on $\left(v_{3}, v_{4}, x_{0}, v_{1}\right)$. This gives the second stated possibility.

We use the notation of Equations (13) and (14) in Section 5. Again, we use the basis $S^{\prime}$, defined in (10). Note that in this section, $v_{k_{3}}=x_{0}$. Moreover, if $k_{3}=3$, then $S_{j}=S_{j}^{\prime}$.

Proposition 6.3. If $v_{2}$ is tight, then one of the following is true:

(1) $\left|v_{3}\right|=4, v_{4}=e_{1}+e_{3}-e_{4}$, and $\left|v_{j}\right|=2$ for all $5 \leq j \leq 4+t, t \geq 0$.

(2) $\left|v_{3}\right|=4, v_{4}=e_{1}+e_{3}-e_{4}, v_{5}=e_{0}+e_{1}+e_{4}-e_{5}$, and $\left|v_{j}\right|=2$ for all $6 \leq j \leq 5+t$, $t \geq 0$.

The corresponding changemaker vectors are:

(1) $\left(1,1,3,5,6^{[t]}\right)$

(2) $\left(1,1,3,5,6,8^{[t+1]}\right)$

Proof. When $v_{2}$ is tight, using Lemma 6.1, the initial segment $\left(\sigma_{0}, \cdots, \sigma_{k_{3}}\right)$ of $\sigma$ is $(1,1,3,5)$. For any $j>3, S_{j}$ will be one of $\emptyset,\{1,2\},\{2,3\},\{1,3\},\{0,1\}$, or $\{0,1,2,3\}$ by Lemma 3.5 and Lemma 4.3. We will first show that $\{1,2\},\{2,3\}$ and $\{0,1,2,3\}$ do not occur. If $S_{j}=\{1,2\}$ for some $j>4$, then $\left\langle v_{j}, v_{1}\right\rangle=-1,\left\langle v_{j}, x_{0}\right\rangle=2$, and $\left\langle v_{j}, v_{2}\right\rangle=0$. Since $\left[x_{0}\right]$ and $\left[v_{1}\right]$ abut $\left[v_{2}\right]$ on opposite ends, and $\left[v_{j}\right]$ abuts both $\left[x_{0}\right]$ and $\left[v_{1}\right]$, the interval $\left[v_{2}\right]$ is contained in the union of $\left[x_{0}\right],\left[v_{j}\right]$, and $\left[v_{1}\right]$. Therefore, $\left|\left[v_{2} \cap v_{j}\right]\right|=\left|v_{2}\right|=6$, so $\left|\left\langle v_{j}, v_{2}\right\rangle\right|=6-\delta\left(\left[v_{j}\right],\left[v_{2}\right]\right) \geq 3$, a contradiction. If $S_{j}=\{2,3\}$, then $\left\langle v_{j}, v_{2}\right\rangle=-1$ but $\left\langle v_{j}, v_{1}\right\rangle=\left\langle v_{j}, x_{0}\right\rangle=0$. To avoid a claw $\left(v_{2}, v_{1}, x_{0}, v_{j}\right)$, then, we must have $\left[v_{2}\right] \pitchfork\left[v_{j}\right]$. Since $v_{j}$ is orthogonal to $x_{0}$, this means that $\delta\left(\left[v_{2}\right],\left[v_{j}\right]\right)=2$, so $\left|v_{j}\right|=\left|\left[v_{j} \cap v_{2}\right]\right|=3$ and $\epsilon_{2} \neq \epsilon_{j}$. Therefore, $v_{j}+v_{2}$ is reducible. Since $j-1 \in \operatorname{supp}^{+}\left(v_{j}\right)$, the only way to have $\left|v_{j}\right|=3$ is to have $j=4$, but then $v_{j}+v_{2}$ is irreducible by Lemma 4.6. If $S_{j}=\{0,1,2,3\},\left\langle v_{j}, v_{2}\right\rangle=2,\left\langle v_{j}, x_{0}\right\rangle=2$, and $\left\langle v_{j}, v_{1}\right\rangle=0$. Also, $\left|\left[v_{2} \cap v_{j}\right]\right|=\left|v_{j}\right| \geq 5$, so in order to have $\left\langle v_{j}, v_{2}\right\rangle=2$ we must have $\epsilon_{j}=\epsilon_{2}$ and $\delta\left(\left[v_{2}\right],\left[v_{j}\right]\right)=3$. By Lemma 3.11, $\left\langle v_{j}, x_{0}\right\rangle=-\left\langle v_{2}, x_{0}\right\rangle= \pm 2$, a contradiction. Therefore, for each $j>3, S_{j}$ is one of $\emptyset,\{0,1\}$ and $\{1,3\}$. Furthermore, if $S_{j}=\{0,1\}$, then $\left\langle v_{j}, x_{0}\right\rangle \neq 0$, so by Corollary 4.16 there is at most one $j$ with $S_{j}=\{0,1\}$.

If the index 4 exists, $3 \in S_{4}$, so $S_{4}=\{1,3\}, v_{4}=e_{1}+e_{3}-e_{4}$, and $\sigma_{4}=6$. If, for some $j>4$, $S_{j}=\{1,3\}$, then also $4 \in \operatorname{supp}^{+}\left(v_{j}\right)$ by Corollary 4.14 . Therefore, $\left|v_{j}\right| \geq 4$ and $\left\langle v_{j}, v_{4}\right\rangle=1$, so $\left[v_{4}\right]$ abuts $\left[v_{j}\right]$. Since $v_{j}$ is orthogonal to $x_{0}, \delta\left(\left[v_{2}\right],\left[v_{j}\right]\right) \leq 2$, so since $\left|v_{j}\right| \geq 4$ and $\left\langle v_{j}, v_{2}\right\rangle=1$ we must have $\left[v_{2}\right] \dagger\left[v_{j}\right]$. Therefore, using Corollary 4.15, either $\left[v_{2}\right]$ and $\left[v_{4}\right]$ are distant or they share a common end, but in either case we cannot have $\left\langle v_{2}, v_{4}\right\rangle=1$. Therefore, there is at most one $j>4$ with $S_{j}=\{0,1\}$, and for all other $i$ we have $S_{i}=\emptyset$. Suppose that for some $j$ we have $S_{j}=\{0,1\}$. It follows from Lemma 4.26 that $\left|v_{i}\right|=2$ when $4<i<j$. By Lemma 4.3, $v_{j}=e_{0}+e_{1}+e_{k}+e_{k+1}+\cdots+e_{j-1}-e_{j}$ for some $4 \leq k<j$, and to avoid a claw $\left(v_{j}, v_{1}, x_{0}, v_{k}\right)$ we must have $k=4$. Therefore, $\left|v_{j}\right|=j-1 \geq 4$. Since $\left\langle v_{j}, v_{2}\right\rangle=3$, we must 
have $\epsilon_{j}=\epsilon_{2}$, and since $\left\langle v_{j}, x_{0}\right\rangle=\left\langle v_{2}, x_{0}\right\rangle=2$ this means that $\delta\left(\left[v_{2}\right],\left[v_{j}\right]\right)=1$. Therefore, $\left|v_{j}\right|=\left\langle v_{j}, v_{2}\right\rangle+1=4$, so $j=5$. This means that $S_{5}$ is either $\emptyset$ or $\{0,1\}$, and $S_{i}=\emptyset$ for $i>5$.

If $S_{5}=\emptyset$, by Lemma 4.26, $\left|v_{i}\right|=2$ when $i \geq 5$. If $S_{5}=\{0,1\}$, we will show that min supp $v_{i} \geq 5$ when $i>5$.

We first claim that $x_{z_{4}} \in\left[v_{2}\right]$. Otherwise, as $\left\langle v_{4}, v_{2}\right\rangle=1$, we get $\left[v_{2}\right] \dagger\left[v_{4}\right]$ and $\epsilon_{2}=-\epsilon_{4}$. We also have $\left\langle v_{2}, v_{1}\right\rangle=-\left\langle v_{4}, v_{1}\right\rangle=1$. Thus we have either $\left[v_{1}\right] \prec\left[v_{2}\right]$ or $\left[v_{1}\right] \prec\left[v_{4}\right]$. If $\left[v_{1}\right] \prec\left[v_{2}\right]$, then $\epsilon_{1}=\epsilon_{2}$ and $\epsilon_{1}=\epsilon_{4}$, a contradiction to $\epsilon_{2}=-\epsilon_{4}$. Similarly, we can rule out $\left[v_{1}\right] \prec\left[v_{4}\right]$. This proves the claim.

Note that $\sigma_{0}=\sigma_{1}$ are the only two 1's in the coordinates of $\sigma$, so there does not exist any norm 2 vector $y \in(\sigma)^{\perp}$ such that $\left\langle y, v_{1}\right\rangle=-1$. Thus $\left[v_{1}\right]$ contains only one vertex which does not neighbor any norm 2 vertex. Since $v_{1} \sim v_{2}$ and $\left\langle v_{1}, x_{0}\right\rangle=0$, [ $\left.v_{1}\right]$ abuts the right end of $\left[v_{2}\right]$. As $x_{z_{4}} \in\left[v_{2}\right]$ and $v_{4} \sim v_{1}, x_{z_{4}}$ is the rightmost high weight vertex in [ $\left.v_{2}\right]$. If min supp $v_{i}=4$ for some $i>5$, then $v_{i} \sim v_{4}$ and $\left|v_{i}\right| \geq 3$. As $\left\langle v_{i}, v_{2}\right\rangle=0, x_{z_{i}}$ is the leftmost high weight vertex to the right of $\left[v_{2}\right]$. So $\left[v_{1}\right]$ is the unique vertex between $x_{z_{4}}$ and $x_{z_{i}}$. We then see that $\left[v_{1}\right]$ and $\left[v_{i}\right]$ abut, which is not possible as $\left\langle v_{1}, v_{i}\right\rangle=0$. This proves that $\min \operatorname{supp} v_{i} \geq 5$ when $i>5$. By Lemma 4.26, $\left|v_{i}\right|=2$ when $i>5$.

Proposition 6.4. If $v_{2}$ is not tight and $\left(\sigma_{0}, \ldots, \sigma_{k_{3}}\right) \neq(1,1,1,2,3)$, then one of the following is true (if only the norm of a standard basis vector is given, it is just right):

(1) $\left|v_{3}\right|=4,\left|v_{4}\right|=3,\left|v_{j}\right|=2$ for $5 \leq j \leq 4+t, v_{5+t}=e_{1}+e_{2}+e_{4}+e_{5}+\cdots+e_{4+t}-e_{5+t}$, $\left|v_{6+t}\right|=3$, and $\left|v_{j}\right|=2$ for $j>6+t(t \geq 0)$.

(2) $\left|v_{3}\right|=4,\left|v_{4}\right|=3$, and $\left|v_{5}\right|=6$.

(3) $\left|v_{3}\right|=4,\left|v_{4}\right|=5$, and $\left|v_{5}\right|=4$.

with corresponding changemaker vectors:

(1) $\left(1,1,1,3,4,4^{[t]}, 4 t+6,(4 t+10)^{[s]}\right), s, t \geq 0$

(2) $(1,1,1,3,4,10)$

(3) $(1,1,1,3,6,10)$

Proof. If $v_{2}$ is not tight and $\left(\sigma_{0}, \ldots, \sigma_{k_{3}}\right) \neq(1,1,1,2,3)$, using Lemma 6.2, it follows that $\left(\sigma_{0}, \cdots, \sigma_{k_{3}}\right)$ is $(1,1,1,3)$. Note that, using Lemmas 3.5 and 4.3 ,

$$
S_{i}=\emptyset,\{1,2\},\{2,3\} \text {, or }\{0,1,2,3\}, \quad \text { when } i \geq 4 \text {. }
$$

Using Lemma 4.7, we get that $S_{4}$ is either $\{2,3\}$ or $\{0,1,2,3\}$, that is, $\sigma_{4}$ is either 4 or 6 .

When $\sigma_{4}=6, v_{4}=e_{0}+e_{1}+e_{2}+e_{3}-e_{4}$. Since $\left\langle v_{4}, x_{0}\right\rangle=2$, using Corollary 4.16 and (19),

$$
S_{i}=\emptyset \text { or }\{2,3\} \quad \text { when } i>4 \text {. }
$$

Since the intersection graph must be connected, there will be some index $j$ for which $S_{j}=$ $\{2,3\}$. Additionally, using Corollary 4.14, we get that $4 \in \operatorname{supp}^{+} v_{j}$, as otherwise $\left\langle v_{j}, v_{4}\right\rangle=2$. It turns out that there is only one such $j$. In fact, if there were two such indices $j_{1}, j_{2}$, then $\{2,3,4\} \subset S_{j_{1}} \cap S_{j_{2}}$, we would have $\left\langle v_{j_{1}}, v_{j_{2}}\right\rangle \geq 2$, a contradiction. We claim that $j=5$. If $j \neq 5$, then $S_{5}=\emptyset$ by (20). Therefore, $\left|v_{5}\right|=2$, so, by Lemma $4.3,4$ cannot be a gappy index for $v_{j}$. This will give us a claw $\left(v_{4}, x_{0}, v_{5}, v_{j}\right)$. This justifies the claim; in particular, $\sigma_{5}=10$. 
If the index 6 existed, by (20) we must have $S_{6}=\emptyset$. Thus, $v_{6}$ is either $e_{4}+e_{5}-e_{6}$ or $e_{5}-e_{6}$. In the first case, there will be a claw $\left(v_{4}, v_{5}, v_{6}, x_{0}\right)$ and in the second case there will be a claw $\left(v_{5}, v_{4}, v_{6}, v_{2}\right)$. So the index 6 does not exist, and we get the third possibility listed in the proposition.

Now, suppose that $\sigma_{4}=4$. If $\sigma_{5} \neq 4,6$, by Lemma 4.7 and (19), $S_{5}$ is either $\{0,1,2,3\}$ or $\{2,3\}$. If $S_{i}=\{0,1,2,3\}$ or $\{2,3\}$ for some $i>5$, we will get a heavy triple $\left(v_{4}, v_{5}, v_{i}\right)$. So $S_{i}=\emptyset$ or $\{1,2\}$ when $i>5$.

If $S_{5}=\{2,3\}$, then $e_{5}=e_{2}+e_{3}+e_{4}-e_{5}$. Since the pairing graph is connected, there exists an index $i>5$ such that $S_{i}=\{1,2\}$. Using the path $v_{i} \sim v_{1} \sim v_{2}$, we will get a heavy triple $\left(v_{4}, v_{5}, v_{i}\right)$.

If $S_{5}=\{0,1,2,3\}, \sigma_{5}=10$. If the index 6 does exist, using Corollary 4.16, $S_{6}=\emptyset$. We will have a claw $\left(v_{4}, v_{2}, v_{5}, v_{6}\right)$ or $\left(v_{5}, x_{0}, v_{4}, v_{6}\right)$, depending on whether or not $4 \in \operatorname{supp}^{+}\left(v_{6}\right)$. So we get the second possibility listed in the proposition.

If $\sigma_{5}=6$, since $\left\langle v_{5}, x_{0}\right\rangle=2$, by Corollary 4.16 and (19) we have $S_{i}=\emptyset$ or $\{2,3\}$ when $i>5$. Assume that there exists $i>5$ such that $S_{i}=\{2,3\}$. Since $\left\langle v_{i}, v_{4}\right\rangle \leq 1,4 \in \operatorname{supp}\left(v_{i}\right)$. Since $\left\langle v_{i}, v_{5}\right\rangle \leq 1,5 \in \operatorname{supp}\left(v_{i}\right)$. We would then have a heavy triple $\left(v_{4}, v_{5}, v_{i}\right)$. So $S_{i}=\emptyset$ whenever $i>5$. If $\left|v_{6}\right|=2$, there will be a claw $\left(v_{5}, v_{1}, x_{0}, v_{6}\right)$. So $v_{6}=e_{4}+e_{5}-e_{6}$. Since $\left\langle v_{5}, x_{0}\right\rangle=2$, $x_{z_{5}}=x_{1}$. Since $v_{5}$ is connected to $v_{4}$ by a path of norm 2 vectors, $x_{z_{4}}$ is the leftmost high weight vertex to the right of $x_{z_{5}}$. Since $v_{4} \sim v_{6}$, by Corollary 4.15, $\left\langle v_{i}, v_{5}\right\rangle=\left\langle v_{i}, v_{4}\right\rangle=0$, whenever $i>6$. We then conclude that $\min \operatorname{supp}\left(v_{i}\right) \geq 6$ when $i>6$. Using Lemma 4.26, we get $\left|v_{i}\right|=2$ when $i>6$. This gives us the case $t=0$ in the first possibility listed in the proposition.

If $\sigma_{5}=4$, since the pairing graph is connected, there must be a unique index $j>4$ for which $\left\langle v_{j}, x_{0}\right\rangle=2$. Then $\sigma_{j}>4$, and $S_{j}$ is either $\{0,1,2,3\}$ or $\{1,2\}$ by (19). Let $t+5$ be the index such that $\sigma_{t+4}=4<\sigma_{t+5}$.

If $S_{j}=\{0,1,2,3\}$, then in order to avoid $\left\langle v_{j}, v_{4}\right\rangle=2$ (which contradicts Corollary 4.14) we must have $4 \in \operatorname{supp}^{+}\left(v_{j}\right)$. Moreover, using Lemma 4.3 , neither of $4,5, \ldots, t+3$ can be a gappy index for $v_{j}$. Hence we get a claw $\left(v_{4}, v_{2}, v_{j}, v_{5}\right)$ as $j>t+4 \geq 5$. That is, we must have $S_{j}=\{1,2\}$.

We claim that $j=t+5$. Suppose for contradiction that $j \neq t+5$. Then, using Corollary 4.16 and (19), $S_{t+5}$ is either $\emptyset$ or $\{2,3\}$. If $S_{t+5}=\{2,3\}$, then there will be a heavy triple $\left(v_{4}, v_{t+5}, v_{j}\right)$, where the paths connecting the three high norm vertices are through $v_{1}$ and/or $v_{2}$. If $S_{t+5}=\emptyset$, set $i=\min \operatorname{supp}\left(v_{t+5}\right)$. Using Lemma 4.3 , none of $4, \cdots, t+3$ can be a gappy index for $v_{t+5}$. Then there will be a claw on either $\left(v_{i}, v_{i-1}, v_{t+5}, v_{i+1}\right)$ or $\left(v_{4}, v_{2}, v_{t+5}, v_{5}\right)$, depending on whether $4<i<t+4$ or $i=4$. (Note that $i \neq t+4$ since $\sigma_{t+5}>4$.) This finishes the proof of the claim, that is, $j=t+5$ and $S_{t+5}=\{1,2\}$.

To avoid a cycle $v_{t+5} \sim v_{4} \sim v_{2} \sim v_{1} \sim v_{t+5}$ of length bigger than 3 (which violates Corollary 4.25), we must have $4 \in \operatorname{supp}^{+}\left(v_{t+5}\right)$. Furthermore, using Lemmas 4.3 and 4.7 , all the indices $5, \cdots, t+4 \in \operatorname{supp}\left(v_{t+5}\right)$, so $\sigma_{t+5}=4 t+6$. For $i>t+5$, using Corollary 4.16 and (19), the set $S_{i}$ is either $\emptyset$ or $\{2,3\}$. If $S_{i}=\{2,3\}$, we will get a heavy triple $\left(v_{i}, v_{4}, v_{t+5}\right)$. This proves that $S_{i}=\emptyset$ whenever $i>t+5$. Set $\ell=\min \operatorname{supp}\left(v_{i}\right)$. If $\ell=t+5$, there will be a claw 
$\left(v_{t+5}, x_{0}, v_{i}, v_{1}\right)$. If $4<\ell<t+4$, there will be a claw $\left(v_{\ell}, v_{\ell-1}, v_{i}, v_{\ell+1}\right)$, and if $\ell=4$ the claw will be on $v_{4}, v_{2}, v_{i}, v_{5}$. Therefore $\ell=t+4$ or $\ell \geq t+6$. In particular, $e_{t+6}=e_{t+4}+e_{t+5}-e_{t+6}$ and $\sigma_{t+6}=4 t+10$. When $i>t+6$, if $\ell=t+4$, we get a heavy triple $\left(v_{i}, v_{4}, v_{t+6}\right)$. So $\ell \geq t+6$ when $i>t+6$. Now we can apply Lemma 4.26 to conclude that $\left|v_{i}\right|=2$ whenever $i>t+6$, and we will get the first possibility listed in the proposition.

Proposition 6.5. If $\left(\sigma_{0}, \ldots, \sigma_{k_{3}}\right)=(1,1,1,2,3), v_{5}=e_{2}+e_{3}+e_{4}-e_{5}$, and $\left|v_{j}\right|=2$ for $j>5$. In this case, $\sigma=\left(1,1,1,2,3,6^{[t]}\right), t \geq 1$.

Proof. Since $4 \in S_{5}^{\prime}, S_{5}^{\prime}=\{2,4\}$ by Lemma 3.5 and Corollary 4.16, so the set $S_{5}$ is equal to either $\{2,4\}$ or $\{2,3,4\}$. If $S_{5}=\{2,4\}$, then there will be a cycle of length 4 on $\left(v_{3}, v_{1}, v_{2}, v_{5}\right)$. Therefore, $S_{5}=\{2,3,4\}$, and so, $\sigma_{5}=6$. There is a path $v_{3} \sim v_{1} \sim v_{2} \sim v_{5}$. For any $i>5$, to avoid a heavy triple $\left(v_{i}, v_{3}, v_{5}\right), v_{i}$ cannot neighbor $v_{1}$ or $v_{2}$. Combined with Lemmas 4.3 and 3.5 and Corollary 4.16, we must have $S_{i}^{\prime}=\emptyset$. If $3 \in S_{i}$, we would have a claw $\left(v_{3}, v_{i}, v_{1}, x_{0}\right)$. So $S_{i}=\emptyset$. By Lemma 4.26, we have $\left|v_{i}\right|=2$ whenever $i>5$.

Now $\sigma=\left(1,1,1,2,3,6^{[t]}\right), t \geq 0$. If $t=0$, then $p=1$, (see Section 8.) So we must have $t \geq 1$.

$$
\text { 7. } k_{1}>1
$$

In the present section we classify all the changemaker C-type lattices that have

$$
x_{0}=e_{0} \pm e_{k_{1}} \pm e_{k_{2}} \pm e_{k_{3}},
$$

where $k_{1}>1$. Using Lemma 3.5, we know that

$$
v_{1}=2 e_{0}-e_{1},
$$

and therefore, $\sigma_{1}=2$ and $\left|v_{1}\right|=5$. We remind the reader that, by Lemma $4.11, v_{1}$ is the only tight vector in the C-type lattices that concern us in this section. We also note that

$$
0 \in \operatorname{supp}\left(v_{k_{1}}\right)
$$

by Lemma 3.5. Compared to Sections 5 and 6, it will take longer to determine the initial segment $\left(\sigma_{0}, \cdots, \sigma_{k_{3}}\right)$ of $\sigma$. We start by specifying the positive integer $k_{1}$.

Lemma 7.1. The segment $\left(\sigma_{0}, \cdots \sigma_{k_{1}}\right)$ is either $(1,2,3)$ or $(1,2,2,3)$. In particular, $k_{1}=2$ or 3 , and $\sigma_{k_{1}}=3$.

Proof. Using Lemma 4.11, we get that $v_{2}$ is either $e_{0}+e_{1}-e_{2}$ or $e_{1}-e_{2}$. In the former case, using Lemma 3.5, we get that $k_{1}=2$, and so $\sigma_{k_{1}}=3$.

Now suppose that $v_{2}=e_{1}-e_{2}$. More generally, suppose that there exists $t \geq 1$ such that $\left(\sigma_{0}, \sigma_{1}, \cdots, \sigma_{t+1}\right)=\left(1,2,2^{[t]}\right)$, and that $\left|v_{t+2}\right|>2$. We will show that $t=1, k_{1}=3$, and that $\sigma_{t+2}$ (or simply $\sigma_{3}$ ) is 3 .

Set $j=\min \operatorname{supp}\left(v_{t+2}\right)$. We argue that $j=0$. (Note that, by Lemma 4.3 , none of $1,2, \cdots, t$ is a gappy index for $v_{t+2}$.) If $1<j<t+1$, there will be a claw on $v_{j}, v_{j-1}, v_{t+2}, v_{j+1}$. If $j=1$, then $\left\langle v_{t+2}, v_{1}\right\rangle=-1$ and $v_{t+2}$ will be orthogonal to $v_{2}$. Then $k_{1}>t+2$ by (22). There will be a claw on $v_{1}, x_{0}, v_{t+2}, v_{2}$, unless $\left[v_{t+2}\right] \pitchfork\left[v_{1}\right],\left|\left[v_{1} \cap v_{t+2}\right]\right|=\left|v_{t+2}\right|=3$, and $\epsilon_{t+2}=-\epsilon_{1}$. 
Thus $v_{1}+v_{t+2}$ is the sum of two distant intervals and so is reducible. Since $\left|v_{t+2}\right|=3$, $v_{t+2}=e_{1}+e_{t+1}-e_{t+2}$, and so $v_{1}+v_{t+2}$ is irreducible by Lemma 4.6 , a contradiction. That is, $j=0$, and that,

$$
v_{t+2}=e_{0}+e_{i}+e_{i+1}+\cdots+e_{t+1}-e_{t+2},
$$

with $i \geq 1$.

Since $0 \in \operatorname{supp}^{+}\left(v_{t+2}\right)$, using Lemma 3.5, we get that $k_{1}=t+2$. Furthermore, we claim that $x_{0}=e_{0}+e_{t+2}+e_{k_{2}}-e_{k_{3}}$. See Proposition 4.9. If $x_{0}=e_{0}-e_{t+2}-e_{k_{2}}+e_{k_{3}}$, then $\left\langle v_{t+2}, x_{0}\right\rangle=2$. Observe that $\left\langle v_{t+2}, v_{1}\right\rangle=1$ or 2 depending on whether or not $i=1$ in (23); in particular, $\left\langle v_{t+2}, v_{1}\right\rangle>0$. Since $\left|v_{t+2} \cap v_{1}\right|=\left|v_{t+2}\right| \geq 3$ and $\delta\left(\left[v_{1}\right],\left[v_{t+2}\right]\right) \leq 3$, using Lemma 3.9, it must be that $\epsilon_{1}=\epsilon_{t+2}$. Since $\left\langle v_{1}, x_{0}\right\rangle=\left\langle v_{t+2}, x_{0}\right\rangle=2,\left[v_{1}\right]$ and $\left[v_{t+2}\right]$ share their left endpoint, and $\delta\left(\left[v_{t+2}\right],\left[v_{1}\right]\right)=1$. Moreover, we must have $\left|v_{t+2}\right|=3$ (as otherwise $\left\langle v_{t+2}, v_{1}\right\rangle>2$ ). That is, $v_{t+2}=e_{0}+e_{t+1}-e_{t+2}$. We have $\left\langle v_{2}, v_{1}\right\rangle=-1$ and $\left\langle v_{2}, x_{0}\right\rangle=0$, so $\left[v_{2}\right]$ abuts the right end of $\left[v_{1}\right]$. Since also $v_{t+2} \sim v_{t+1},\left|v_{i}\right|=2$ for $i \in\{2, \cdots, t+1\}$,

$$
v_{2} \sim v_{3} \sim \cdots \sim v_{t+1}
$$

the interval $\left[v_{1}\right]$ is a subset of the union of the $\left[v_{j}\right]$ for $j \in\{2, \cdots, t+2\}$, which in turn implies that $\left|v_{1}\right|=\left|v_{t+2}\right|=3$, a contradiction. This shows that

$$
x_{0}=e_{0}+e_{t+2}+e_{k_{2}}-e_{k_{3}} .
$$

We now argue that $1 \notin \operatorname{supp}\left(v_{t+2}\right)$. Suppose for contradiction that $1 \in \operatorname{supp}\left(v_{t+2}\right)$. Using (23), we get that $\left|v_{t+2}\right| \geq 4,\left\langle v_{1}, v_{t+2}\right\rangle=1$ and $\left\langle v_{2}, v_{t+2}\right\rangle=0$. To avoid a claw on $v_{1}, x_{0}, v_{t+2}, v_{2}$, we must have $\left[v_{t+2}\right] \pitchfork\left[v_{1}\right]$. This implies that $\delta\left(\left[v_{1}\right],\left[v_{t+2}\right]\right)=2$. Using Lemma 3.9 and that $\left|v_{t+2}\right| \geq 4$, we see that $\left|\left\langle v_{1}, v_{t+2}\right\rangle\right| \geq 2$, a contradiction. That is, in (23), we must have $i>1$.

We claim that $i=2$. If $2<i<t+1$, there will be a claw on $v_{i}, v_{i-1}, v_{t+2}, v_{i+1}$. If $i=t+1$ (and $i>2)$, to avoid a claw on $v_{1}, x_{0}, v_{t+2}, v_{2}$, it must be that $\left[v_{t+2}\right] \pitchfork\left[v_{1}\right]$, and so $\delta\left(\left[v_{t+2}\right],\left[v_{1}\right]\right)=2$. To get $\left\langle v_{t+2}, v_{1}\right\rangle=2$, however, it must be $\left|v_{t+2}\right|=4$ which contradicts $i=t+1$. Therefore, in (23), we have $i=2$. In particular, $v_{2} \sim v_{t+2}$.

Finally, we argue that $t=1$. If $t>1$, we must have $v_{1} \sim v_{t+2}$ as otherwise we get a claw $\left(v_{2}, v_{1}, v_{t+2}, v_{3}\right)$. That is, $\left[v_{t+2}\right]$ abuts $\left[v_{1}\right]$. Therefore, to fulfill $\left\langle v_{t+2}, v_{1}\right\rangle=2,\left[v_{t+2}\right] \prec\left[v_{1}\right]$, and that $\left|v_{t+2}\right|=3$, which contradicts $t>1$ and (23). So $t=1$ as desired.

As part of the proof of Lemma 7.1, we showed that $x_{0}=e_{0}+e_{k_{1}}+e_{k_{2}}-e_{k_{3}}$ when $k_{1}=3$. Indeed, this is the case also when $k_{1}=2$.

Lemma 7.2. Let $k_{1}>1$. Then $x_{0}=e_{0}+e_{k_{1}}+e_{k_{2}}-e_{k_{3}}$.

Proof. We only need to show this for $k_{1}=2$. Suppose for contradiction $x_{0}=e_{0}-e_{2}-e_{k_{2}}+e_{k_{3}}$ (see Proposition 4.9). Note that $v_{2}=e_{0}+e_{1}-e_{2}$, and therefore, $\left\langle v_{2}, x_{0}\right\rangle=2=\left\langle v_{1}, x_{0}\right\rangle$, and $\left\langle v_{2}, v_{1}\right\rangle=1$. Since $\left|v_{2}\right|=3$, using Lemma 3.9, we see that $\epsilon_{1}=\epsilon_{2}$ and $\delta\left(\left[v_{1}\right],\left[v_{2}\right]\right)=2$. Since $\left\langle\left[v_{2}\right], x_{0}\right\rangle=\left\langle\left[v_{1}\right], x_{0}\right\rangle= \pm 2,\left[v_{1}\right],\left[v_{2}\right]$ share their left end point, so we cannot have $\delta\left(\left[v_{1}\right],\left[v_{2}\right]\right)=2$, a contradiction.

Now we proceed to determine the changemaker vectors. As in Section 5, we use the notation of (13) and (14). Also, we use the basis $S^{\prime}$, defined in (10), where $v_{k_{3}}$ is replaced by $x_{0}$. 
7.1. $\boldsymbol{k}_{1}=\mathbf{2}$. This subsection is devoted to classifying the changemaker C-type lattices with

$$
x_{0}=e_{0}+e_{2}+e_{k_{2}}-e_{k_{3}} .
$$

Recall that the changemaker starts with $(1,2,3)$. We have

$$
\left\langle v_{1}, v_{2}\right\rangle=1, \quad\left\langle v_{2}, x_{0}\right\rangle=0 .
$$

Lemma 7.3. The intervals $\left[v_{2}\right]$ and $\left[v_{1}\right]$ are consecutive with $\epsilon_{2}=-\epsilon_{1}$.

Proof. Using (25) and Lemma 3.9, either $\left[v_{2}\right] \pitchfork\left[v_{1}\right],\left|\left[v_{2}\right] \cap\left[v_{1}\right]\right|=\left|\left[v_{2}\right]\right|=3, \delta\left(\left[v_{2}\right],\left[v_{1}\right]\right)=2$, and $\epsilon_{2}=\epsilon_{1}$, or $\left[v_{2}\right] \dagger\left[v_{1}\right]$, and $\epsilon_{2}=-\epsilon_{1}$. In the former case, $v_{2}-v_{1}$ is the sum of two distant intervals, and so is reducible. However, we have $v_{2}=e_{0}+e_{1}-e_{2}$, and so $v_{2}-v_{1}$ is irreducible by Lemma $4.6(2)$.

Lemma 7.4. There does not exist an index $j>3, j \neq k_{3}$, such that $\operatorname{supp}\left(v_{j}\right) \cap\{0,1,2\}=\{1\}$.

Proof. Otherwise, we will have $\left\langle v_{j}, v_{1}\right\rangle=-\left\langle v_{j}, v_{2}\right\rangle=-1$. We also have $\left\langle v_{j}, x_{0}\right\rangle=0$ by Lemma 3.5. By Lemma 7.3, $\left[v_{j}\right]$ and $\left[v_{1}\right]$ share their right endpoint, so $\delta\left(\left[v_{j}\right],\left[v_{1}\right]\right)=1$. By Lemma $3.9,\left|\left\langle v_{1}, v_{j}\right\rangle\right|=\left|v_{j}\right|-1>1$, a contradiction.

Lemma 7.5. $\sigma_{3} \in\{3,4\}$. Furthermore, if $\sigma_{3}=4$ then $\left[v_{3}\right]$ and $\left[v_{1}\right]$ share their left endpoint, and that $\epsilon_{3}=\epsilon_{1}$.

Proof. All the possibilities for $\sigma_{3}$ lie in $\{3,4,5,6\}$. If $\sigma_{3}=5$, we get that $v_{3}=e_{1}+e_{2}-e_{3}$. So $\left\langle v_{3}, v_{1}\right\rangle=-1$ and $v_{3}$ is orthogonal to $v_{2}$. By Lemma $3.5, k_{2}=3$ and $\left\langle v_{3}, x_{0}\right\rangle=0$. Using Lemma 7.3 , we know that $\left[v_{2}\right]$ abuts $\left[v_{1}\right]$, and therefore, there will be a claw on $v_{1}, x_{0}, v_{3}, v_{2}$, unless $\left[v_{3}\right] \pitchfork\left[v_{1}\right],\left|\left[v_{1}\right] \cap\left[v_{3}\right]\right|=\left|v_{3}\right|=3$, and $\epsilon_{3}=-\epsilon_{1}$. Thus $v_{1}+v_{3}$ is the sum of two distant intervals and so is reducible. However, $v_{3}+v_{1}$ is irreducible by Lemma 4.6, a contradiction. If $\sigma_{3}=6$, we see that $v_{3}=e_{0}+e_{1}+e_{2}-e_{3}$ (and, in particular, $\left|v_{3}\right|=4$ ). This implies that $\left\langle v_{3}, x_{0}\right\rangle=2$ and $\left\langle v_{1}, v_{3}\right\rangle=1$. The latter will only be possible if both $\delta\left(\left[v_{1}\right],\left[v_{3}\right]\right)=3$ and $\epsilon_{1}=\epsilon_{3}$, a contradiction to Lemma 3.11.

If $\sigma_{3}=4$, we have $v_{3}=e_{0}+e_{2}-e_{3}$. Using Lemma 3.9, the second statement of the lemma is immediate because $\left\langle v_{3}, v_{1}\right\rangle=\left\langle v_{3}, x_{0}\right\rangle=\left\langle v_{1}, x_{0}\right\rangle=2$ and $\left|v_{3}\right|=3$.

Lemma 7.6. If $0 \in \operatorname{supp}\left(v_{j}\right)$ and $2 \notin \operatorname{supp}\left(v_{j}\right)$ for some $j>3$ and $j \neq k_{3}$, then $\left[v_{j}\right],\left[v_{1}\right]$ share their right endpoint, and $v_{j}=e_{0}+e_{j-1}-e_{j}$. Moreover, there exists at most one such $j$.

Proof. We have $1 \notin \operatorname{supp}\left(v_{j}\right)$, otherwise $\left\langle v_{2}, v_{j}\right\rangle=2$, a contradiction to Corollary 4.14. So $\left\langle v_{1}, v_{j}\right\rangle=2$. Since $\left\langle v_{j}, v_{2}\right\rangle=1,\left[v_{j}\right]$ and $\left[v_{2}\right]$ are consecutive by Corollary 4.15. It follows from Lemma 7.3 that $\left[v_{j}\right]$ and $\left[v_{1}\right]$ share their right endpoint, and so $\delta\left(\left[v_{j}\right],\left[v_{1}\right]\right)=1$. Then, to get $\left\langle v_{1}, v_{j}\right\rangle=2$, we must have $\left|v_{j}\right|=3$ and $v_{j}=e_{0}+e_{j-1}-e_{j}$. Lastly, there exists at most one such $j$ by Corollary 4.15 .

Proposition 7.7. If $\sigma_{3}=3$, the initial segment $\left(\sigma_{0}, \cdots, \sigma_{k_{3}}\right)$ of $\sigma$ is $(1,2,3,3,7)$.

Proof. Suppose that $\sigma_{3}=3$ (see Lemma 7.5). This implies that $k_{2}=3$ (Lemma 3.5). Using Equation (24), we see that $\sigma_{k_{3}}=7$. We claim that $k_{3}=k_{2}+1=4$. If $k_{3} \neq 4$, by Lemma 3.5, $\sigma_{4} \in\{4,6\}$. Suppose $\sigma_{4}=4$, or equivalently, $v_{4}=e_{0}+e_{3}-e_{4}$. This gives us that $\left\langle v_{4}, x_{0}\right\rangle=2$ 
and $\left\langle v_{4}, v_{2}\right\rangle=1$. By Lemma 7.3, the interval $\left[v_{1}\right]$ will be a subset of $\left[v_{4}\right] \cup\left\{x_{0}\right\}$, which implies that $\left|v_{1}\right|=3$, a contradiction. Suppose $\sigma_{4}=6$, or equivalently, $v_{4}=e_{2}+e_{3}-e_{4}$. Then there will be a claw $\left(v_{2}, v_{1}, v_{4}, v_{3}\right)$. This justifies the claim, that is, $\sigma_{4}=7$ and $k_{3}=4$.

Proposition 7.8. If $\sigma_{3}=4$, the initial segment $\left(\sigma_{0}, \cdots, \sigma_{k_{3}}\right)$ of $\sigma$ is either $(1,2,3,4,5,9)$ or $\left(1,2,3,4^{[s]}, 4 s+3,4 s+7\right), s \geq 1$.

Proof. All the possibilities for $\sigma_{4}$ lie in $\{4,5,6,7,8,9,10\}$. We first argue that $\sigma_{4} \notin\{6,8,9,10\}$. Suppose $\sigma_{4}=6$, then $v_{4}=e_{1}+e_{3}-e_{4}$, contradicting Lemma 7.4. If $\sigma_{4}=10$, then $v_{4}$ will have nonzero inner product with $v_{2}$ and $v_{3}$. Using Lemmas 7.5 and 7.3 , the interval $\left[v_{1}\right]$ equals the union of $\left[v_{3}\right]$ and $\left[v_{4}\right]$, that is, $\left|v_{1}\right|=6$, a contradiction. If $\sigma_{4}=8$, then both the unbreakable vectors $v_{3}$ and $v_{4}$ will have nonzero inner product with $x_{0}$, contradicting Corollary 4.16. When $\sigma_{4}=9, v_{4}=e_{1}+e_{2}+e_{3}-e_{4}$. Notice that $\left\langle v_{4}, v_{1}\right\rangle=-1$ while $v_{4}$ is orthogonal to $x_{0}$. The latter gives us that $\delta\left(\left[v_{4}\right],\left[v_{1}\right]\right) \leq 2$. Therefore, given that $\left|v_{4}\right|=4$, we must have $\left[v_{4}\right]$ and $\left[v_{2}\right]$ share their left endpoint by Lemma 7.3, a contradiction to Corollary 4.15. Therefore $\sigma_{4} \in\{4,5,7\}$.

Suppose that $\sigma_{4}=5$, that is, $v_{4}=e_{0}+e_{3}-e_{4}$. Using Lemma 3.5, $k_{2}=4$, and so $\sigma_{k_{3}}=9$ by Equation (24). Since $\left\langle v_{3}, x_{0}\right\rangle=2,\left\langle v_{5}, x_{0}\right\rangle=0$ by Corollary 4.16, unless $k_{3}=5$. Since $4 \in \operatorname{supp}\left(v_{5}\right)$, we get that $k_{3}=5$.

Let $s \geq 1$ be the integer satisfying that $\sigma_{3}=\cdots=\sigma_{s+2}=4$, and that $\sigma_{s+3}>4$. By Lemma 3.5, $k_{2} \geq s+3$. Set $j=\min \operatorname{supp}\left(v_{s+3}\right)<s+2$. If $3<j<s+2$, there will be a claw $\left(v_{j}, v_{j-1}, v_{s+3}, v_{j+1}\right)$, and if $j=3$, the claw will be $\left(v_{3}, x_{0}, v_{s+3}, v_{4}\right)$. If $j=1$, then $2 \in \operatorname{supp}\left(v_{s+3}\right)$ by Lemma 7.4. Thus $\left|v_{s+3}\right| \geq 4$. Since $\left\langle v_{s+3}, x_{0}\right\rangle=0, \delta\left(\left[v_{s+3}\right],\left[v_{1}\right]\right) \leq 2$. Then

$$
\left|\left\langle v_{s+3}, v_{1}\right\rangle\right| \geq 4-2 \geq 2,
$$

a contradiction. Lastly, suppose $j=0$. By Corollary 4.16, $\left\langle v_{s+3}, x_{0}\right\rangle=0$, it must be the case that $2 \notin \operatorname{supp}\left(v_{s+3}\right)$. By Lemma 7.6, $v_{s+3}=e_{0}+e_{s+2}-e_{s+3}$. If $s=1$ and $\sigma_{4}=5$, this case was discussed in the previous paragraph. However, if $s>1$, then $\left\langle v_{s+3}, v_{3}\right\rangle=1$, and so $\left[v_{1}\right]$ will be the union of $\left[v_{3}\right]$ and $\left[v_{s+3}\right]$ by Lemma 7.5 and Lemma 7.6. Since $\left|v_{3}\right|=3$, to get $\left|v_{1}\right|=5$, it must be that $\left|v_{s+3}\right|=4$, a contradiction. So we are left with the case $j=2$.

Note that $\left\langle v_{s+3}, v_{2}\right\rangle=-1$, and $v_{s+3}$ is orthogonal to $v_{1}$ and $x_{0}$, so $\left[v_{s+3}\right]$ is distant from $\left[v_{1}\right]$ by Lemma 7.3. Using Lemma 7.5, we get that $v_{s+3}$ is orthogonal to $v_{3}$, and so $3 \in \operatorname{supp}\left(v_{s+3}\right)$. By Lemma 4.3, we get that $4, \cdots, s+1 \in \operatorname{supp}\left(v_{s+3}\right)$. That is, $\sigma_{s+3}=4 s+3$, and that $k_{2}=s+3$. Using Equation (24), we get that $\sigma_{k_{3}}=4 s+7$. With the same argument as in the case $\sigma_{4}=5$, we get that $k_{3}=k_{2}+1=s+4$. This recovers the case $\sigma_{4}=7$ when $s=1$.

Proposition 7.9. If $\left(\sigma_{0}, \cdots, \sigma_{k_{3}}\right)=(1,2,3,4,5,9)$, then $n+1=k_{3}$ (i.e. $v_{k_{3}}$ is the last standard basis vector).

Proof. We claim that the index 6 does not exist. Suppose for contradiction that it exists. Since $5 \in S_{6}^{\prime}$, then $S_{6}^{\prime}$ must be one of $\{4,5\},\{2,5\}$, or $\{0,5\}$ (Lemma 3.5 and Corollary 4.16).

By Lemma 7.6, the intervals $\left[v_{4}\right]$ and $\left[v_{1}\right]$ share their right endpoint, and $S_{6}^{\prime} \neq\{0,5\}$.

Suppose that $S_{6}^{\prime}=\{4,5\}$ or $\{2,5\}$, then $\left\langle v_{6}, x_{0}\right\rangle=0$. We have that one of $\left\langle v_{6}, v_{4}\right\rangle$ and $\left\langle v_{6}, v_{3}\right\rangle$ is zero and the other one is nonzero, depending on whether or not $3 \in S_{6}$. By Lemma 7.3 
and Corollary 4.15, $\left[v_{6}\right]$ and $\left[v_{1}\right]$ are not consecutive. Using Lemma 7.5 and the fact that $\left[v_{4}\right]$ and $\left[v_{1}\right]$ share their right endpoint, we conclude that $\left[v_{6}\right] \subset\left[v_{1}\right]$ and $\delta\left(\left[v_{6}\right],\left[v_{1}\right]\right) \leq 2$. Since $\left|v_{6}\right| \geq 3$, we must have $\left\langle v_{6}, v_{1}\right\rangle \neq 0$. That is, $1 \in \operatorname{supp}\left(v_{6}\right)$, and so $\left|v_{6}\right| \geq 4$. Using Lemmas 7.3, 7.5 and Corollary 4.15, $\left[v_{1}\right]$ will have all the high weight vertices of $\left[v_{3}\right],\left[v_{6}\right]$, and $\left[v_{4}\right]$, and so, $\left|v_{1}\right| \geq 6$, a contradiction. This proves the claim.

Proposition 7.10. When $\left(\sigma_{0}, \cdots, \sigma_{k_{3}}\right)=(1,2,3,3,7)$, there exists $s \geq 0$, such that $v_{s+5}=$ $e_{3}+\cdots+e_{s+4}-e_{s+5}, v_{5}=e_{0}+e_{4}-e_{5}$ if $s>0$, and $\left|v_{j}\right|=2$ for $5<j<s+5$ and $j>s+5$. In this case, $\sigma=\left(1,2,3,3,7,8^{[s]}, 8 s+10^{[t]}\right)(s, t \geq 0)$.

Proof. First suppose that $\sigma_{5} \neq 10$. Since $k_{3}=4 \in S_{5}^{\prime}$, the set $S_{5}^{\prime}$ is either $\{0,4\},\{3,4\}$, or $\{0,2,3,4\}$ (Lemmas 3.5 and 4.3). If $S_{5}^{\prime}=\{3,4\}$, as $\sigma_{5} \neq 10$, we must have $1 \in S_{5}$, a contradiction to Lemma 7.4. If $S_{5}^{\prime}=\{0,2,3,4\}$, then $\left\langle v_{1}, v_{5}\right\rangle>0$. Since $\left|v_{5}\right| \geq 5$ and $\delta\left(\left[v_{1}\right],\left[v_{5}\right]\right) \leq 3$, we have $\epsilon_{1}=\epsilon_{5}$. Since $\left\langle v_{1}, v_{5}\right\rangle \leq 2$, and that $\left|v_{5}\right| \geq 5$, we must have $\delta\left(\left[v_{5}\right],\left[v_{1}\right]\right)=3$. Since $\epsilon_{1}=\epsilon_{5}$, by Lemma 3.11, $\left\langle v_{5}, x_{0}\right\rangle=-\left\langle v_{1}, x_{0}\right\rangle= \pm 2$, which is not true. Therefore, $S_{5}^{\prime}=\{0,4\}$ and $v_{5}=e_{0}-e_{4}+e_{5}$ by Lemma 7.6.

We claim that if $S_{j} \neq \emptyset$ for some $j>5$, then $S_{j}=\{3,4\}$. Assume that $S_{j} \neq \emptyset$. By Lemmas 3.5 and $4.3, S_{j}^{\prime}$ is one of $\emptyset,\{0,3\},\{0,4\},\{2,3\},\{3,4\}$, and $\{0,2,3,4\}$. If $S_{j}^{\prime}=\emptyset$, then $S_{j}=\{1\}$, contradicting Lemma 7.4. Since $S_{5}^{\prime}=\{0,4\}, S_{j}^{\prime} \neq\{0,3\}$ or $\{0,4\}$ by Lemma 7.6. If $S_{j}^{\prime}=\{2,3\}$, then $\left\langle v_{j}, x_{0}\right\rangle=2$. Since $\delta\left(\left[v_{j}\right],\left[v_{1}\right]\right) \leq 3,\left|v_{j}\right| \geq 4$, we have $\left\langle v_{j}, v_{1}\right\rangle \neq 0$. Since $0 \notin \operatorname{supp}\left(v_{j}\right)$, we must have $1 \in \operatorname{supp}\left(v_{j}\right)$, and so $\left|v_{j}\right| \geq 5$. Using Lemma 3.9, we get $\left|\left\langle v_{j}, v_{1}\right\rangle\right|>1$, contradicting the fact that $\left\langle v_{1}, v_{j}\right\rangle=-1$. If $S_{j}^{\prime}=\{0,2,3,4\}$, then we have $\left\langle v_{j}, x_{0}\right\rangle=2$ and $\left|v_{j}\right| \geq 6$. Thus $x_{1}=x_{z_{j}}$ is contained in $\left[v_{1}\right]$. However, $\left|v_{1}\right|=5<6=\left|v_{j}\right|$, a contradiction. So $S_{j}^{\prime}=\{3,4\}$. Using Lemma 7.4 , we conclude that $1 \notin S_{j}$. So $S_{j}=\{3,4\}$.

If $S_{j}=\emptyset$ for all $j>5$, it follows from Lemma 4.26 that $\left|v_{j}\right|=2$ whenever $j>5$. Now assume that $S_{j} \neq \emptyset$ for some $j>5$. Let $s+5$ be the smallest such $j$. Then $S_{s+5}=\{3,4\}$ by the earlier discussion. We also know that $\left|v_{i}\right|=2$ for any $5<i<s+5$ by Lemma 4.26 . If $5 \notin \operatorname{supp}\left(v_{s+5}\right)$, then $\left\langle v_{s+5}, v_{5}\right\rangle \neq 0$ and $\left\langle v_{s+5}, v_{3}\right\rangle \neq 0$, and so there will be a cycle $\left(v_{s+5}, v_{3}, v_{2}, v_{5}\right)$ of length bigger than 3: see Figure 8. Thus $5 \in \operatorname{supp}\left(v_{s+5}\right)$, and as a result $6, \cdots, s+4 \in \operatorname{supp}\left(v_{s+5}\right)$ by Lemma 4.3. Therefore, $\sigma_{s+5}=8 s+10$.

Note that, $S_{j}=\emptyset$ when $j>s+5$. Otherwise, by the earlier discussion, $S_{j}=\{3,4\}$, and we would have a heavy triple $\left(v_{j}, v_{s+5}, v_{2}\right)$. Given $j>s+5$, let $\ell=\min \operatorname{supp}\left(v_{j}\right) \geq 5$. Note that

$$
v_{5} \sim v_{6} \sim \cdots \sim v_{s+4},
$$

$\left[v_{5}\right]$ and $\left[v_{1}\right]$ share their right endpoint, $\left\langle v_{i}, v_{1}\right\rangle=0$ and $\left|v_{i}\right|=2$ when $5<i<s+5$, so $\left[v_{i}\right] \subset\left[v_{1}\right]$ when $5 \leq i<s+5$. If $\ell \leq s+4$, then $\left\langle v_{j}, v_{\ell}\right\rangle \neq 0$. Thus $\left[v_{j}\right] \cap\left[v_{1}\right] \neq \emptyset$. Note also that $\delta\left(\left[v_{j}\right],\left[v_{1}\right]\right) \leq 2$ since $v_{j}$ is orthogonal to $x_{0}$. Since $\left|v_{j}\right| \geq 3$, we get that $\left|\left\langle v_{j}, v_{1}\right\rangle\right|>0$, a contradiction. Thus we have proved that $\min \operatorname{supp}\left(v_{j}\right) \geq s+5$ when $j>s+5$. It follows from Lemma 4.26 that $\left|v_{j}\right|=2$ when $j>s+5$.

Finally suppose that $\sigma_{5}=10$. Assume that there exists $\ell>5$ such that $S_{\ell} \neq \emptyset$. By Lemmas 3.5 and $4.3, S_{\ell}^{\prime}$ is one of $\emptyset,\{0,3\},\{0,4\},\{2,3\},\{3,4\}$, and $\{0,2,3,4\}$. If $S_{\ell}^{\prime}=\emptyset$, then $S_{\ell}=\{1\}$, contradicting Lemma 7.4. By Lemma 7.6, $S_{\ell}^{\prime} \neq\{0,3\}$ or $\{0,4\}$. Suppose $S_{\ell}^{\prime}=\{2,3\}$. If $1 \notin \operatorname{supp}\left(v_{\ell}\right)$, there will be a claw $\left(v_{2}, v_{1}, v_{\ell}, v_{3}\right)$. If $1 \in \operatorname{supp}\left(v_{\ell}\right)$, then $\left|\left\langle v_{\ell}, v_{1}\right\rangle\right|=1$. By 

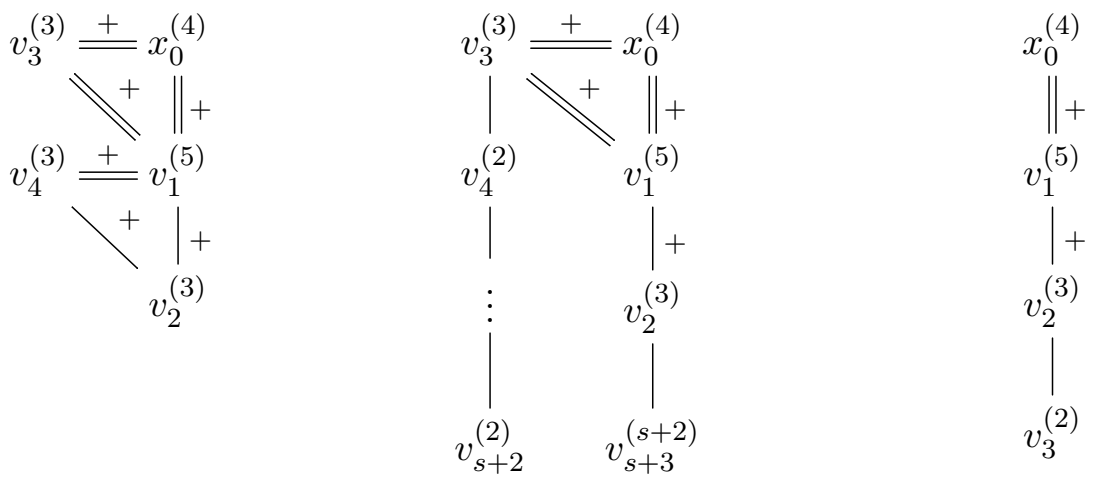

Figure 8. Pairing graphs when $\left(\sigma_{0}, \cdots, \sigma_{k_{3}}\right)$ is $(1,2,3,4,5,9)$ (left), $\left(1,2,3,4^{[s]}, 4 s+3,4 s+7\right)$ (center), or $(1,2,3,3,7)$ (right).

Lemma 7.3, $\left[v_{\ell}\right]$ and $\left[v_{1}\right]$ are not consecutive. Since $\delta\left(\left[v_{\ell}\right],\left[v_{1}\right]\right) \leq 3$ and $\left|v_{\ell}\right| \geq 5$, we get $\left|\left\langle v_{\ell}, v_{1}\right\rangle\right| \geq 2$, a contradiction. If $S_{\ell}^{\prime}=\{3,4\}$, there will be a heavy triple $\left(v_{5}, v_{\ell}, v_{2}\right)$. If $S_{\ell}^{\prime}=\{0,2,3,4\}$, then $\left|v_{\ell}\right| \geq 6$ and $\left\langle v_{\ell}, x_{0}\right\rangle=2$, so $x_{z_{\ell}}=x_{1}$. Thus $\left|\left[v_{1}\right]\right| \geq\left|\left[v_{\ell}\right]\right| \geq 6$, a contradiction. So we proved that $S_{\ell}=\emptyset$ whenever $\ell>5$. It follows from Lemma 4.26 that $\left|v_{j}\right|=2$ when $j>5$.

Proposition 7.11. If $\left(\sigma_{0}, \cdots \sigma_{k_{3}}\right)=\left(1,2,3,4^{[s]}, 4 s+3,4 s+7\right), s>0$, then $v_{s+5}=e_{s+3}+$ $e_{s+4}-e_{s+5}$, and $\left|v_{j}\right|=2$ for $j>s+5$. In this case, $\sigma=\left(1,2,3,4^{[s]}, 4 s+3,4 s+7,(8 s+10)^{[t]}\right)$ $(s>0, t \geq 0)$.

Proof. Suppose that $\ell>s+4$ is an index such that $S_{\ell} \neq \emptyset$. We will prove that $\ell=s+5$ and $v_{s+5}=e_{s+3}+e_{s+4}-e_{s+5}$. Our conclusion then follows from Lemma 4.26.

Step 1. $S_{\ell}^{\prime}$ must be either $\emptyset$ or $\{s+3, s+4\}$.

Using Lemma 4.3 and Corollary $4.16, S_{\ell}^{\prime}$ is either $\emptyset,\{0, s+4\},\{2, s+4\}$, or $\{s+3, s+4\}$. Suppose $S_{\ell}^{\prime}=\{0, s+4\}$, by Lemma 7.6, $v_{\ell}=e_{0}+e_{s+4}-e_{s+5},\left[v_{\ell}\right]$ and $\left[v_{1}\right]$ share their right endpoint. As $\left\langle v_{\ell}, v_{3}\right\rangle \neq 0,\left[v_{1}\right]$ equals the union of $\left[v_{3}\right]$ and $\left[v_{\ell}\right]$ by Lemma 7.5, i.e. $\left|v_{1}\right|=4$, a contradiction. Suppose $S_{\ell}^{\prime}=\{2, s+4\}$. If $1 \notin S_{\ell}$, as $s+3 \notin S_{\ell},\left\langle v_{e l l}, v_{s+3}\right\rangle \neq 0$, there will be a heavy triple $\left(v_{\ell}, v_{s+3}, v_{2}\right)$. If $1 \in S_{\ell}$ (and consequently, $\left|v_{\ell}\right| \geq 4$ ), then there will be a claw $\left(v_{1}, x_{0}, v_{\ell}, v_{2}\right)$, unless $\left[v_{\ell}\right] \pitchfork\left[v_{1}\right]$. If $\left[v_{\ell}\right] \pitchfork\left[v_{1}\right]$, however, we get $\delta\left(\left[v_{\ell}\right],\left[v_{1}\right]\right)=2$, and so $\left|\left\langle v_{\ell}, v_{1}\right\rangle\right| \geq 2$, a contradiction to the fact that $\left\langle v_{\ell}, v_{1}\right\rangle=-1$.

Step 2. If $S_{\ell}^{\prime}=\emptyset$ or $\{s+3, s+4\}$, then $S_{\ell}=S_{\ell}^{\prime}$. In particular, $S_{s+5}=\{s+3, s+4\}$.

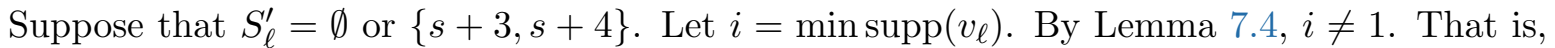
$\left\langle v_{\ell}, v_{1}\right\rangle=0$. Also, note that $v_{\ell}$ is orthogonal to $x_{0}$, and so $\delta\left(\left[v_{\ell}\right],\left[v_{1}\right]\right) \leq 2$. If $3 \leq i \leq s+2$, since $v_{3} \sim v_{4} \sim \cdots \sim v_{i} \sim v_{\ell}$, using Lemmas 7.5 and 7.3, $x_{z_{\ell}} \in\left[v_{1}\right]$. Therefore, $\left\langle v_{1}, v_{\ell}\right\rangle \neq 0$, a contradiction. So $i \geq s+3$ and hence $S_{\ell}=S_{\ell}^{\prime}$. Clearly, $S_{s+5}=\{s+3, s+4\}$ by Lemma 4.7.

Step 3. If $S_{\ell}=\{s+3, s+4\}$, then $\ell=s+5$. 
Assume that $\ell>s+5$ and $S_{\ell}=\{s+3, s+4\}$, then we have a heavy triple $\left(v_{s+3}, v_{s+5}, v_{\ell}\right)$.

7.2. $k_{1}=3$. In this subsection we focus on the changemaker C-type lattices with

$$
x_{0}=e_{0}+e_{3}+e_{k_{2}}-e_{k_{3}} .
$$

Recall that the changemaker starts with $(1,2,2,3)$.

Lemma 7.12. The intervals $\left[v_{3}\right]$ and $\left[v_{1}\right]$ share their right endpoint and $\epsilon_{3}=\epsilon_{1}$. Moreover, $\left[v_{2}\right]$ abuts the right endpoint of $\left[v_{1}\right]$ and $\left[v_{3}\right]$.

Proof. Since $\left|v_{3}\right|=3$ and $\left\langle v_{1}, v_{3}\right\rangle=2$, from Lemma 3.9, it must be the case that $\epsilon_{1}=\epsilon_{3}$ and $\delta\left(\left[v_{1}\right],\left[v_{3}\right]\right)=1$. The first statement of the lemma is now immediate because $v_{3}$ is orthogonal to $x_{0}$. Since $\left\langle v_{2}, v_{1}\right\rangle \neq 0$ and $\left\langle v_{2}, x_{0}\right\rangle=0,\left[v_{2}\right]$ abuts the right endpoint of $\left[v_{1}\right]$.

Corollary 7.13. Suppose that there exists a vector $v_{j}$ such that $j>3, j \neq k_{3}$, and $\left\langle v_{j}, v_{1}\right\rangle=2$. Then $j=4$, and that $v_{4}=e_{0}+e_{3}-e_{4}$.

Proof. Suppose that $j$ is such an index. Therefore, $0 \in \operatorname{supp}^{+}\left(v_{j}\right)$ and $1 \notin \operatorname{supp}^{+}\left(v_{j}\right)$. (This, in particular, implies that $\left.\left|v_{j}\right| \geq 3\right)$. We claim that $\left\langle v_{j}, x_{0}\right\rangle \neq 0$. Otherwise, assume $\left\langle v_{j}, x_{0}\right\rangle=0$. Since $\left\langle v_{j}, v_{1}\right\rangle=2, x_{z_{j}} \in\left[v_{1}\right]$. Using Lemma 7.12 and Corollary 4.15, $\left[v_{1}\right]$ contains at least 3 high weight vertices $x_{1}, x_{z_{j}}, x_{z_{3}}$, and $\delta\left(\left[v_{j}\right],\left[v_{1}\right]\right)=2$. Since $\left|v_{1}\right|=5$, we have $\left|x_{z_{j}}\right|=3$, so by Lemma 3.9 we have $\left|\left\langle v_{j}, v_{1}\right\rangle\right|=1$, a contradiction. This justifies the claim, and therefore, $\left\langle v_{j}, x_{0}\right\rangle=2$. Since $\left|v_{j}\right| \geq 3$ and $\delta\left(\left[v_{1}\right],\left[v_{j}\right]\right) \leq 3$, to get $\left\langle v_{j}, v_{1}\right\rangle=2$, we must have $\epsilon_{1}=\epsilon_{j}$. Thus, $\delta\left(\left[v_{j}\right],\left[v_{1}\right]\right)=1$ and $\left|v_{j}\right|=3$. That is, $v_{j}=e_{0}+e_{j-1}-e_{j}$. We now argue that $j=4$. Suppose for contradiction that $j>4$. Thus $\left\langle v_{j}, v_{3}\right\rangle=1$. Using Lemma 7.12, we get that the interval $\left[v_{1}\right]$ equals the union of $\left[v_{j}\right]$ and $\left[v_{3}\right]$. Since $\left|v_{j}\right|=\left|v_{3}\right|=3$, we get that $\left|v_{1}\right|=4$, which is a contradiction.

Lemma 7.14. Let $v_{j}$ be a vector such that $j>3, j \neq k_{3}$. Then $\left\langle v_{j}, v_{1}\right\rangle \in\{0,2\}$. As a result, $\min \operatorname{supp}\left(v_{j}\right) \geq 2$ unless $j=4$ and $v_{4}=e_{0}+e_{3}-e_{4}$.

Proof. Assume that $\left\langle v_{j}, v_{1}\right\rangle \notin\{0,2\}$, then $\operatorname{supp}\left(v_{j}\right) \cap\{0,1\}=\{1\}$ or $\{0,1\}$. By Lemma 4.3, $2 \in \operatorname{supp}\left(v_{j}\right)$. If $0 \in \operatorname{supp}\left(v_{j}\right)$, since $\left|\left\langle v_{j}, v_{3}\right\rangle\right| \leq 1$ by Corollary 4.14 , we have $3 \in \operatorname{supp}\left(v_{j}\right)$. Thus $\left|v_{j}\right| \geq 5$. Since $\left\langle x_{0}, v_{j}\right\rangle=2, x_{z_{j}}=x_{1}$. By Corollary 4.15 and Lemma $7.12, x_{z_{j}} \neq x_{z_{3}}$. So

$$
5=\left|v_{1}\right| \geq\left|x_{z_{j}}\right|+\left|x_{z_{3}}\right|-2 \geq 5+1,
$$

a contradiction.

We have shown that $0 \notin \operatorname{supp}\left(v_{j}\right)$. If $3 \notin \operatorname{supp}\left(v_{j}\right)$, then $j>4$ and $\left|v_{j}\right| \geq 4$. As $\left\langle v_{j}, v_{3}\right\rangle=$ 1 , using Corollary 4.15, $\left[v_{j}\right]$ and $\left[v_{3}\right]$ are consecutive. By Lemma 7.12 and the fact that $\left\langle v_{j}, v_{2}\right\rangle=0$ we conclude that $\left[v_{j}\right] \subset\left[v_{1}\right]$. Since $\left\langle v_{j}, x_{0}\right\rangle=0,\left[v_{1}\right]$ contains at least three high weight vertices: $x_{1}, x_{z_{j}}, x_{z_{3}}$. This is impossible as $\left|v_{1}\right|=5$ and $\left|v_{j}\right| \geq 4$.

Now we have $\operatorname{supp}\left(v_{j}\right) \cap\{0,1,2,3\}=\{1,2,3\}$, so $\left\langle v_{j}, v_{3}\right\rangle=0$. By Lemma 4.7, $\left|v_{j}\right| \geq 5$ unless $j=4$. By Lemma 7.12 and the fact that $\left\langle v_{j}, v_{1}\right\rangle \neq 0$ we conclude that $\left[v_{j}\right] \subset\left[v_{1}\right]$. So $\left[v_{1}\right]$ contains at least two high weight vertices: $x_{z_{j}}, x_{z_{3}}$. It follows that $\left|v_{j}\right| \leq 4$. So $j=4$ and $\left|v_{4}\right|=e_{1}+e_{2}+e_{3}-e_{4}$. Since $\left|v_{4}\right|=4,\left[v_{1}\right]$ contains exactly two high weight vertices, so $x_{1}$ must be $x_{z_{4}}$. So $\left\langle v_{4}, x_{0}\right\rangle \neq 0$, which is not possible. This shows that $\left\langle v_{j}, v_{1}\right\rangle \in\{0,2\}$. 
If $\min \operatorname{supp}\left(v_{j}\right)<2$, then $\left\langle v_{j}, v_{1}\right\rangle \neq 0$. We must have $\left\langle v_{j}, v_{1}\right\rangle=2$, so $j=4$ and $v_{4}=e_{0}+e_{3}-e_{4}$ by Corollary 7.13 .

Lemma 7.15. Let $v_{j}$ be a vector such that $j>4, j \neq k_{3}$. Then $\operatorname{supp}\left(v_{j}\right) \cap\{0,1,2,3\} \neq\{2\}$ or $\{3\}$.

Proof. Assume that $\operatorname{supp}\left(v_{j}\right) \cap\{0,1,2,3\}$ contains only one element which is 2 or 3 . Then $\left|v_{j}\right| \geq 3,\left\langle v_{j}, v_{3}\right\rangle \neq 0$ while $\left\langle v_{j}, v_{1}\right\rangle=0$. By Lemma 7.12, $\left[v_{j}\right]$ abuts the left endpoint of $\left[v_{3}\right]$, so $\left[v_{j}\right] \subset\left[v_{1}\right]$. Since $\left|v_{j}\right| \geq 3$ and $\delta\left(\left[v_{j}\right],\left[v_{1}\right]\right) \leq 3$, using Lemma 3.9, we get that $\left\langle v_{j}, v_{1}\right\rangle \neq 0$ unless $\left|v_{j}\right|=\delta\left(\left[v_{j}\right],\left[v_{1}\right]\right)=3$. However, if $\delta\left(\left[v_{j}\right],\left[v_{1}\right]\right)=3,\left[v_{1}\right]$ is contained in the union of $\left[v_{j}\right],\left[v_{3}\right]$ and $\left\{x_{0}\right\}$. Since $\left|v_{j}\right|=\left|v_{3}\right|=3$, we have $\left|v_{1}\right|=4$, a contradiction.

Lemma 7.16. $\sigma_{4} \in\{3,4,5\}$. Furthermore, if $\sigma_{4}=3$ then $\left[v_{4}\right]$ abuts the left endpoint of $\left[v_{3}\right]$. If $\sigma_{4}=4$ then $\left[v_{4}\right]$ and $\left[v_{1}\right]$ share their left endpoint.

Proof. If $\min \operatorname{supp}\left(v_{4}\right)<2$, using Lemma 7.14, $\sigma_{4}=4$. By Lemma 4.7, if $\min \operatorname{supp}\left(v_{4}\right) \geq 2$, $v_{4}=e_{2}+e_{3}-e_{4}$ or $e_{3}-e_{4}$. So $\sigma_{4}=5$ or 3 .

When $\sigma_{4}=3,\left[v_{4}\right]$ abuts $\left[v_{3}\right]$ and $\left\langle v_{4}, v_{1}\right\rangle=0$. By Lemma 7.12, $\left[v_{4}\right]$ abuts the left endpoint of $\left[v_{3}\right]$. When $\sigma_{4}=4,\left\langle v_{4}, v_{1}\right\rangle=2=\left\langle v_{4}, x_{0}\right\rangle$. So $\delta\left(\left[v_{4}\right],\left[v_{1}\right]\right)=1$ by Lemma 3.9. Thus $\left[v_{4}\right]$ and $\left[v_{1}\right]$ share their left endpoint by Lemma 7.12.

Proposition 7.17. If $\sigma_{4}=3$, the initial segment $\left(\sigma_{0}, \cdots, \sigma_{k_{3}}\right)$ of $\sigma$ is $(1,2,2,3,3,7)$.

Proof. Suppose that $\sigma_{4}=3$ (see Lemma 7.16). This implies that $k_{2}=4$ (Lemma 3.5). Using Equation (26), we get that $\sigma_{k_{3}}=7$. If $k_{3} \neq 5$, using Lemma 3.5 and Lemma 7.14, we must have $S_{5} \supset\{3,4\}$. By Lemma 7.15 , we have $2 \in S_{5}$, so $\left\langle v_{5}, x_{0}\right\rangle=2$ and $v_{5} \sim v_{2}$. By Lemma 7.12, $\left[v_{1}\right]$ is contained in the union of $x_{0},\left[v_{5}\right],\left[v_{2}\right]$. So $\left|v_{1}\right|=\left|v_{5}\right|=4$, which is not possible.

Proposition 7.18. If $\sigma_{4} \neq 3$, the initial segment $\left(\sigma_{0}, \cdots, \sigma_{k_{3}}\right)$ of $\sigma$ is $\left(1,2,2,3,4^{[s]}, 4 s+\right.$ $5,4 s+9), s \geq 0$.

Proof. Suppose that $\sigma_{4} \neq 3$ (see Lemma 7.16). Furthermore, let $s \geq 0$ satisfy that $\sigma_{i}=4$ for any $4 \leq i<s+4$, and that $\sigma_{s+4}>4$. We have $k_{2} \geq s+4$ by Lemma 3.5. Set $j=\min \operatorname{supp}\left(v_{s+4}\right)<s+3$. Then $j \geq 2$ by Lemma 7.14. Also, $j \neq 3$ by Lemma 7.15. If $4<j<s+3$, we will get a claw $\left(v_{j}, v_{j-1}, v_{s+4}, v_{j+1}\right)$, and if $j=4$, the claw will be on $v_{4}, x_{0}, v_{s+4}, v_{5}$. This proves that $j=2$. By Lemma 7.15, $3 \in \operatorname{supp}\left(v_{s+4}\right)$.

We will show that $\sigma_{s+4}=4 s+5$. If $s=0, v_{4}=e_{2}+e_{3}-e_{4}$, and we are done. If $s>0$, since $2,3 \in \operatorname{supp}\left(v_{s+4}\right),\left|v_{s+4}\right| \geq 4$. Also, $v_{s+4}$ must be orthogonal to $v_{4}$, as otherwise, using Lemmas 7.16 and 7.12 , all the three intervals $\left[v_{4}\right],\left[v_{s+4}\right]$, and $\left[v_{3}\right]$ will be subsets of $\left[v_{1}\right]$, which implies that $\left|v_{1}\right| \geq 6$, a contradiction. That is, $4 \in \operatorname{supp}\left(v_{s+4}\right)$. Using Lemma $4.3, v_{s+4}$ is just right and $\sigma_{s+4}=4 s+5$.

Using Lemma 3.5, we see that $k_{2}=s+4$. By Equation (26), we have $\sigma_{k_{3}}=4 s+9$. Note that $k_{2} \in \operatorname{supp}\left(v_{k_{2}+1}\right)$. Since the unbreakable vector $v_{4}$ has nonzero inner product with $x_{0}$, using Corollary 4.16, we get that $k_{3}=k_{2}+1$. 

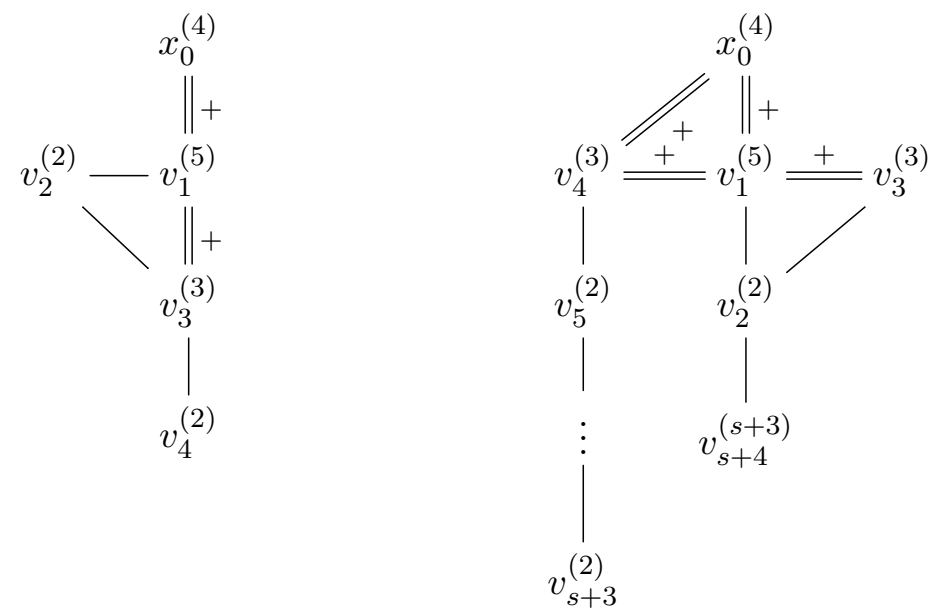

Figure 9. Pairing graphs when $\left(\sigma_{0}, \cdots, \sigma_{k_{3}}\right)$ is $(1,2,2,3,3,7)$ (left) or $\left(1,2,2,3,4^{[s]}, 4 s+5,4 s+9\right), s>0$ (right).

Proposition 7.19. If $\left(\sigma_{0}, \cdots, \sigma_{k_{3}}\right)=(1,2,2,3,3,7)$, then $n+1=k_{3}$ (i.e. $v_{k_{3}}$ is the last standard basis vector).

Proof. We claim that the index $k_{3}+1$ (that is, 6) does not exist. Using Lemmas 3.5, 4.7, 4.3 , and 7.14, $S_{6}^{\prime}=\{4,5\}$. Then $\left\langle v_{6}, v_{4}\right\rangle \neq 0$, and also $v_{6}$ is orthogonal to $x_{0}$. Using Lemmas 7.16 and 7.12 , we must have $\left[v_{6}\right] \subset\left[v_{1}\right]$ which implies that $\left\langle v_{6}, v_{1}\right\rangle \neq 0$ since $\left|v_{6}\right| \geq 3$. This contradicts Lemma 7.14.

Proposition 7.20. If $\left(\sigma_{0}, \cdots, \sigma_{k_{3}}\right)=\left(1,2,2,3,4^{[s]}, 4 s+5,4 s+9\right), s \geq 0$, then $v_{s+6}=$ $e_{s+4}+e_{s+5}-e_{s+6}$ if it exists, and $\left|v_{i}\right|=2$ for $i>s+6$. In this case, $\sigma=\left(1,2,2,3,4^{[s]}, 4 s+\right.$ $\left.5,4 s+9,(8 s+14)^{[t]}\right), t \geq 0$.

Proof. Suppose that $\ell>k_{3}=s+5$ is an index such that $S_{\ell} \neq \emptyset$. We will prove that $\ell=s+6$ and $S_{\ell}=\{s+4, s+5\}$. This, together with Lemma 4.26, will imply our desired result.

By Lemmas 3.5, 7.14, and Corollary 4.16, $S_{\ell}^{\prime}$ is one of $\emptyset,\{3,4\},\{3,5\}$ and $\{4,5\}$ if $s=0$, and one of $\emptyset,\{3, s+5\}$, and $\{s+4, s+5\}$ if $s>0$. Let $j=\min \operatorname{supp}\left(v_{\ell}\right)$, then $j \geq 2$ by Lemma 7.14. Also, $j \neq 3$ by Lemma 7.15.

If $s=0$ and $S_{\ell}^{\prime}=\{3,4\}$, we have $\left\langle v_{\ell}, x_{0}\right\rangle=2$ and $\left|v_{\ell}\right| \geq 4$, so $x_{1} \in\left[v_{\ell}\right]$. Using Lemma 3.9, we get $\left\langle v_{\ell}, v_{1}\right\rangle \neq 0$, a contradiction.

If $S_{\ell}^{\prime}=\{3, s+5\}$, to avoid $\left\langle v_{\ell}, v_{s+4}\right\rangle>1,2 \notin \operatorname{supp}\left(v_{\ell}\right)$. Thus, $j=3$, which is impossible.

Having proved $S_{\ell}^{\prime}=\emptyset$ or $\{s+4, s+5\}$, we claim that $S_{\ell}=S_{\ell}^{\prime}$. First, $j \neq 2$ by Lemma 7.15. So our claim holds when $s=0$. When $s>0$, if $4 \leq j<s+3$, we have a claw $\left(v_{j}, v_{j-1}, v_{j+1}, v_{\ell}\right)$. If $j=s+3,\left\langle v_{\ell}, v_{s+3}\right\rangle \neq 0$. By Lemma 7.16, $\left[v_{4}\right]$ and $\left[v_{1}\right]$ share their left endpoint. Since 
$\left|v_{5}\right|=\cdots=\left|v_{s+3}\right|=2$ and $v_{4} \sim v_{5} \sim \cdots \sim v_{s+3}$, we have $\left[v_{\ell}\right] \subset\left[v_{1}\right]$ by Lemma 7.12. Thus $\left\langle v_{\ell}, v_{1}\right\rangle \neq 0$ by Lemma 3.9, a contradiction. So our claim is proved.

Now by Lemma $4.7, s+5 \in S_{s+6}$. So $S_{s+6}=\{s+4, s+5\}$ by the results in the previous two paragraphs. If there was $\ell>s+6$ satisfying $S_{\ell}=\{s+4, s+5\}$, we would have a heavy triple $\left(v_{s+4}, v_{s+6}, v_{\ell}\right)$. Thus $S_{\ell}=\emptyset$ whenever $\ell>s+6$.

\section{Determining $p$ AND $q$}

In Sections 5, 6, and 7, we have classfied all the $(n+1)$-dimensional C-type lattices that are isomorphic to changemaker lattices. In the present section, we list all the corresponding prism manifolds $P(p, q)$. To do so, we start with the refined basis $S^{\prime}=\left\{v_{1}, \cdots, v_{n+1}\right\} \backslash\left\{v_{k_{3}}\right\} \cup\left\{x_{0}\right\}$ as defined in (10). The first step is changing the basis into the vertex basis $\left\{x_{0}, x_{1}, \cdots, x_{n}\right\}$. We then recover the $a_{i}$ from the norms of vertex basis elements. By using Equation (1), we obtain $p$ and $q$.

Example 8.1. We present an example that clarifies how $(p, q)$ is computed in Proposition 5.6. The changemaker is

$$
\left(1,1,2^{[s]}, 2 s-1,2 s+1\right), s=n-2 \geq 2 .
$$

Let $S^{\prime}$ denote the modified standard basis for the changemaker lattice $L=(\sigma)^{\perp}$. It is straightforward to check that

$$
\left\{x_{0}\right\} \cup\left\{-v_{2}, \cdots,-v_{s+1}, v_{3}+\cdots+v_{s+2}, v_{1}\right\}
$$

forms the vertex basis $S^{*}$. Also, the vertex norms are

$$
\left\{3,2^{[s-1]}, s+1,2\right\} \text {. }
$$

Using Lemma 2.4 together with Equation (1), we have

$$
\frac{2 q-p}{q-p}=\left[3,2^{[s-1]}, s+1,2\right]=\frac{4 s^{2}+3}{2 s^{2}-s+2} .
$$

In particular, $p=2 s-1$ and $q=2 s^{2}+s+1$. We see that $q=\frac{1}{2}\left(p^{2}+3 p+4\right), p \geq 3$.

Similar computations give prism manifolds $P(p, q)$, with $q>p$, so that each falls into one of the families in Table 1. We denote the set of such prism manifolds $\mathcal{P}_{q>p}^{+}$. Here we divide the families so that each changemaker vector corresponds to a unique family. In some cases there are prism manifolds that correspond to more than one family in Table 1. For instance, it is straightforward to check that $P(5,22)$ belongs to both Families 5 and 1A. The detailed correspondence between the changemaker vectors and $P(p, q)$ can be found in Table 3. Note that the positive integer $p$ is always odd.

\section{PRism MANifolds REALIZABle BY SURGERY ON KNOTS IN $S^{3}$}

Table 1 gives a list of all prism manifolds $P(p, q)$, with $q>p$, that can possibly be realized by surgery on knots in $S^{3}$. In $\left[\mathrm{BHM}^{+} 16\right.$, Table 2], a list of realizable prism manifolds $P(p, q)$ with $q>0$ is provided. It is straightforward to verify that the manifolds in Table 1 coincide 
TABLE 1. $\mathcal{P}_{q>p}^{+}$, table of $P(p, q)$ that are realizable, $q>p$

\begin{tabular}{|c|c|c|}
\hline Type & $P(p, q)$ & $\begin{array}{l}\text { Range of parameters } \\
(p \text { and } r \text { are always odd, } p>1)\end{array}$ \\
\hline $1 \mathrm{~A}$ & $P\left(p, \frac{1}{2}\left(p^{2}+3 p+4\right)\right)$ & \\
\hline 1B & $P\left(p, \frac{1}{22}\left(p^{2}+3 p+4\right)\right)$ & $\begin{array}{l}p \equiv 5 \text { or } 3(\bmod 22) \\
p \neq 3,5\end{array}$ \\
\hline 2 & $P\left(p, \frac{1}{|4 r+2|}\left(r^{2} p-1\right)\right)$ & $\begin{aligned} r & \equiv-1(\bmod 4) \\
p & \equiv-2 r+3(\bmod 4 r+2) \\
r & \neq-5,-1,3\end{aligned}$ \\
\hline 3A & $P\left(p, \frac{1}{2 r}(p-1)(p-4)\right)$ & $\begin{array}{l}p \equiv 1(\bmod 2 r) \\
p \neq 2 r+1 \\
r \geq 5\end{array}$ \\
\hline 3B & $P\left(p, \frac{1}{2 r}(p-1)(p-4)\right)$ & $\begin{array}{l}p \equiv r+4(\bmod 2 r) \\
p>r+4 \\
r \geq 1\end{array}$ \\
\hline 4 & $P\left(p, \frac{1}{2 r^{2}}\left((2 r+1)^{2} p-1\right)\right)$ & $\begin{array}{l}p \equiv-4 r+1\left(\bmod 2 r^{2}\right) \\
r \neq 1,-1\end{array}$ \\
\hline 5 & $P\left(p, \frac{1}{r^{2}-2 r-1}\left(r^{2} p-1\right)\right)$ & $\begin{array}{l}r>1 \\
p \equiv-2 r+5\left(\bmod r^{2}-2 r-1\right)\end{array}$ \\
\hline
\end{tabular}

Sporadic $P(11,19), P(13,34)$

with those of $\left[\mathrm{BHM}^{+} 16\right.$, Table 2] with $q>p$. That is, Table 1 is a complete list of prism manifolds $P(p, q)$, with $q>p$, arising from surgery on knots in $S^{3}$. 
9.1. Prism manifolds corresponding to more than one changemaker vector. As we pointed out in Section 8, some of the prism manifolds in Table 1 correspond to distinct changemaker vectors. In this subsection, we address this by providing distinct knots corresponding to such prism manifolds. Our strategy is as follows: let $\sigma$ be a changemaker vector whose orthogonal complement is isomorphic to $C(p, q)$ for some $p$ and $q$. Let $\sigma$ correspond to a knot $K$ in $S^{3}$ on which surgery results in $P(p, q)$. Using Lemma 2.7, we compute the Alexander polynomial $\Delta_{K}(T)$. Then we exhibit a $\mathrm{P} / \mathrm{SF}$ knot $K_{\sigma}$ that admits a surgery to $P(p, q)$. By directly computing $\Delta_{K_{\sigma}}(T)$ we show that the two Alexander polynomials coincide. That is, $K_{\sigma}$ matches with $\sigma$. See $\left[\mathrm{BHM}^{+} 16\right.$, Section 13.2]. The parameters beneath the $\mathrm{P} / \mathrm{SF}$ knots in Table 2 are explained in $\left[\mathrm{BHM}^{+} 16\right]$.

\subsection{Proof of the main results.}

Proof of Theorem 1.4. If $C(p, q)$ is isomorphic to a changemaker lattice $L$, then it belongs to one of the families enumerated in Sections 5, 6, and 7. Following Section 8, we can find a pair $\left(p^{\prime}, q^{\prime}\right)$ such that $L$ is isomorphic to $C\left(p^{\prime}, q^{\prime}\right)$, and $P\left(p^{\prime}, q^{\prime}\right) \in \mathcal{P}_{q>p}^{+}$. Now, Proposition 3.16 finishes the proof.

Proof of Theorem 1.1. Suppose $P(p, q) \cong S_{4 q}^{3}(K)$, it follows from Theorem 1.3 and Theorem 1.4 that $P(p, q)$ belongs to one of the six families in Table 1 and $P(p, q) \cong S_{4 q}^{3}\left(K_{0}\right)$ for some Berge-Kang knot $K_{0}$. To get the result about $\widehat{H F K}$, we note that $K$ and $K_{0}$ correspond to the same changemaker vector. Using Lemma 2.7, we know that $\Delta_{K}=\Delta_{K_{0}}$, so $\widehat{H F K}(K) \cong \widehat{H F K}\left(K_{0}\right)$ by [OS05a, Theorem 1.2]. 
TABLE 2. Prism manifolds $P(p, q)$ corresponding to more than one changemaker

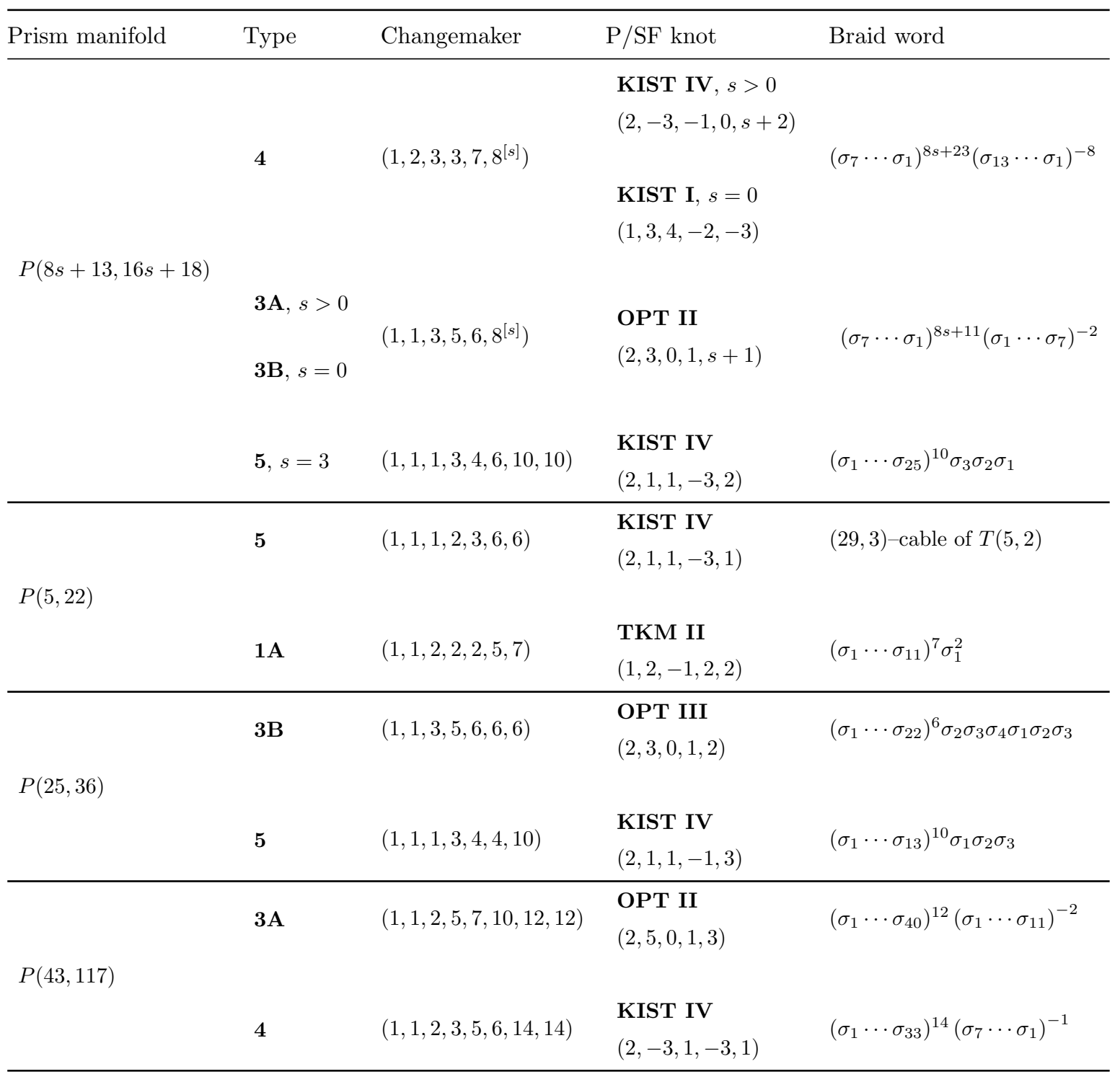


TABLE 3. C-type changemakers and the corresponding prism manifolds, Part I

\begin{tabular}{|c|c|c|}
\hline Prop. & Changemaker vector & Vertex basis (with $x_{0}$ omitted) $\left\{x_{1}, \cdots, x_{n}\right\}$ \\
\hline \multirow[t]{2}{*}{5.6} & $\begin{array}{l}\left(1,1,2^{[s]}, 2 s-1,2 s+1\right) \\
s \geq 2\end{array}$ & $\left\{-v_{2}, \cdots,-v_{s+1}, v_{[3, s+2]}, v_{1}\right\}$ \\
\hline & $\begin{array}{l}\left(1,1,2^{[s]}, 2 s+1,2 s+3,4 s+4,8 s+10\right) \\
s \geq 1\end{array}$ & $\left\{-v_{2}, \cdots,-v_{s+1},-v_{s+5}, v_{s+4}, v_{s+2}, v_{1}\right\}$ \\
\hline \multirow[t]{4}{*}{5.7} & $\begin{array}{l}\left(1,1,2^{[s]}, 2 s+1,2 s+3,4 s+6,8 s+10\right) \\
s \geq 1\end{array}$ & $\left\{-v_{2}, \cdots,-v_{s+1},-v_{s+4}, v_{s+5}, v_{s+2}, v_{1}\right\}$ \\
\hline & $\begin{array}{l}\left(1,1,2,3,5,8^{[s]}, 8 s+6,(8 s+14)^{[t]}\right) \\
s \geq 1\end{array}$ & $\left\{-v_{2}, v_{s+5}, v_{1},-v_{3}-v_{1},-v_{5}, \cdots,-v_{s+4},-v_{s+6}, \cdots,-v_{s+t+5}\right\}$ \\
\hline & $\left(1,1,2,3,5,6,14^{[t]}\right)$ & $\left\{-v_{2}, v_{1}+v_{5},-v_{1},-v_{3},-v_{6}, \cdots,-v_{t+5}\right\}$ \\
\hline & $\begin{array}{l}\left(1,1,2^{[s]}, 2 s+3,2 s+5,(4 s+6)^{[t]}\right) \\
s, t \geq 1\end{array}$ & $\left\{-v_{2}, \cdots,-v_{s+1}, v_{[1, s+1]}+v_{[s+4, s+t+3]}-v_{s+2},-v_{s+t+3}, \cdots,-v_{s+4},-v_{1}\right\}$ \\
\hline \multirow[t]{3}{*}{5.9} & $\begin{array}{l}\left(1,1,2^{[s]}, 2 s+3,2 s+5\right) \\
s \geq 1\end{array}$ & $\left\{-v_{2}, \cdots,-v_{s+1}, v_{[1, s+1]}-v_{s+2},-v_{1}\right\}$ \\
\hline & $\begin{array}{l}\left(1,1,2^{[s]}, 2 s+3,2 s+5,4 s+6,(4 s+8)^{[t]}\right) \\
s, t \geq 1\end{array}$ & $\left\{-v_{2}, \ldots,-v_{s+1},-v_{s+5}, \ldots,-v_{s+t+4}, v_{[1, s+1]}+v_{[s+4, s+t+4]}-v_{s+2},-v_{s+4},-v_{1}\right\}$ \\
\hline & $\begin{array}{l}\left(1,1,3,5,6^{[t]}\right) \\
t \geq 1\end{array}$ & $\left\{v_{1}+v_{[4, t+3]}-v_{2},-v_{t+3}, \ldots,-v_{4},-v_{1}\right\}$ \\
\hline \multirow[t]{3}{*}{6.3} & $(1,1,3,5)$ & $\left\{-v_{2}, v_{1}\right\}$ \\
\hline & $\left(1,1,3,5,6,8^{[t+1]}\right)$ & $\left\{-v_{5}, \cdots,-v_{t+5}, v_{1}+v_{[4, t+5]}-v_{2},-v_{4},-v_{1}\right\}$ \\
\hline & $\left(1,1,1,3,4,4^{[t]}, 4 t+6,(4 t+10)^{[s]}\right)$ & $\left\{-v_{t+5},-v_{1},-v_{2},-v_{4}, \cdots,-v_{t+4},-v_{t+6}, \cdots,-v_{t+s+5}\right\}$ \\
\hline \multirow[t]{2}{*}{6.4} & $(1,1,1,3,4,10)$ & $\left\{-v_{5}, v_{4}, v_{2}, v_{1}\right\}$ \\
\hline & $(1,1,1,3,6,10)$ & $\left\{-v_{4}, v_{5}, v_{2}, v_{1}\right\}$ \\
\hline 6.5 & $\begin{array}{l}\left(1,1,1,2,3,6^{[t]}\right) \\
t \geq 1\end{array}$ & $\left\{-v_{3},-v_{1},-v_{2},-v_{5}, \cdots,-v_{t+4}\right\}$ \\
\hline 7.9 & $(1,2,3,4,5,9)$ & $\left\{-v_{3}, v_{[3,4]}-v_{1},-v_{4}, v_{2}\right\}$ \\
\hline \multirow[t]{2}{*}{7.10} & $\begin{array}{l}\left(1,2,3,3,7,8^{[s]},(8 s+10)^{[t]}\right) \\
s \geq 1\end{array}$ & $\left\{v_{[5, s+4]}-v_{1},-v_{s+4}, \cdots,-v_{5}, v_{2}, v_{3}, v_{s+5}, \cdots, v_{s+t+4}\right\}$ \\
\hline & $\left(1,2,3,3,7,10^{[t]}\right)$ & $\left\{-v_{1}, v_{2}, v_{3}, v_{5}, \cdots, v_{t+4}\right\}$ \\
\hline 7.11 & $\begin{array}{l}\left(1,2,3,4^{[s]}, 4 s+3,4 s+7,(8 s+10)^{[t]}\right) \\
s \geq 1\end{array}$ & $\left\{-v_{3}, \cdots,-v_{s+2}, v_{[3, s+2]}-v_{1}, v_{2}, v_{s+3}, v_{s+5}, \cdots, v_{s+t+4}\right\}$ \\
\hline 7.19 & $(1,2,2,3,3,7)$ & $\left\{v_{[3,4]}-v_{1},-v_{4},-v_{3},-v_{2}\right\}$ \\
\hline \multirow[t]{2}{*}{7.20} & $\begin{array}{l}\left(1,2,2,3,4^{[s]}, 4 s+5,4 s+9,(8 s+14)^{[t]}\right) \\
s \geq 1\end{array}$ & $\left\{-v_{4}, \cdots,-v_{s+3}, v_{[3, s+3]}-v_{1},-v_{3},-v_{2},-v_{s+4},-v_{s+6}, \cdots,-v_{s+t+5}\right\}$ \\
\hline & $\left(1,2,2,3,5,9,14^{[t]}\right)$ & $\left\{v_{3}-v_{1},-v_{3},-v_{2},-v_{4},-v_{6}, \cdots,-v_{t+5}\right\}$ \\
\hline
\end{tabular}


TABLE 3. C-type changemakers and the corresponding prism manifolds, Part II

\begin{tabular}{|c|c|c|c|}
\hline Prop. & Vertex norms $\left\{a_{1}, \ldots, a_{n}\right\}$ & Prism manifold parameters & $\mathcal{P}_{q>p}^{+}$type \\
\hline \multirow{2}{*}{5.6} & \multirow{2}{*}{$3,2^{[s-1]}, s+1,2$} & $p=2 s-1$ & \multirow{2}{*}{$1 \mathrm{~A}$} \\
\hline & & $q=2 s^{2}+s+1$ & \\
\hline \multirow{10}{*}{5.7} & \multirow{2}{*}{$3,2^{[s-1]}, 5,3, s+2,2$} & $p=22 s+25$ & \multirow{2}{*}{ 1B } \\
\hline & & $q=22 s^{2}+53 s+32$ & \\
\hline & \multirow{2}{*}{$3,2^{[s-1]}, 4,4, s+2,2$} & $p=22 s+27$ & \multirow{2}{*}{ 1B } \\
\hline & & $q=22 s^{2}+57 s+37$ & \\
\hline & \multirow{3}{*}{$3, s+3,2,3,3,2^{[s-1]}, 3,2^{[t-1]}$} & $r=2 s+3$ & \multirow{3}{*}{4} \\
\hline & & $p=2 r^{2}(t+1)-4 r+1$ & \\
\hline & & $q=(2 r+1)^{2}(t+1)-8 r-6$ & \\
\hline & \multirow{3}{*}{$3,3,2,3,4,2^{[t-1]}$} & $r=3$ & \multirow{3}{*}{4} \\
\hline & & $p=18 t+7$ & \\
\hline & & $q=49 t+19$ & \\
\hline \multirow{9}{*}{5.9} & \multirow{3}{*}{$3,2^{[s-1]}, 4,2^{[t-1]}, s+3,2$} & $r=2 t+1$ & \multirow{3}{*}{$3 B$} \\
\hline & & $p=2 r(s+1)+r+4$ & \\
\hline & & $q=\frac{1}{2}(2 r s+3(r+1))(2 s+3)$ & \\
\hline & \multirow{3}{*}{$3,2^{[s-1]}, s+5,2$} & $r=1$ & \multirow{3}{*}{ 3B } \\
\hline & & $p=2 s+7$ & \\
\hline & & $q=(s+3)(2 s+3)$ & \\
\hline & \multirow{3}{*}{$3,2^{[s-1]}, 3,2^{[t-1]}, 3, s+3,2$} & $r=2 t+3$ & \multirow{3}{*}{$3 \mathbf{A}$} \\
\hline & & $p=2 r(s+2)+1$ & \\
\hline & & $q=(s+2)(2 r(s+2)-3)$ & \\
\hline \multirow{9}{*}{6.3} & \multirow{3}{*}{$5,2^{[t-1]}, 3,2$} & $r=2 t+1$ & \multirow{3}{*}{$3 B$} \\
\hline & & $p=6 t+7$ & \\
\hline & & $q=9 t+9$ & \\
\hline & \multirow{3}{*}{6,2} & $r=1$ & \multirow{3}{*}{ 3B } \\
\hline & & $p=7$ & \\
\hline & & $q=9$ & \\
\hline & \multirow{3}{*}{$4,2^{[t]}, 3,3,2$} & $r=2 t+5$ & \multirow{3}{*}{$3 \mathbf{A}$} \\
\hline & & $p=8 t+21$ & \\
\hline & & $q=16 t+34$ & \\
\hline
\end{tabular}


TABLE 3. C-type changemakers and the corresponding prism manifolds, Part III

\begin{tabular}{|c|c|c|c|}
\hline Prop. & Vertex norms $\left\{a_{1}, \ldots, a_{n}\right\}$ & Prism manifold parameters & $\mathcal{P}_{q>p}^{+}$type \\
\hline \multirow{7}{*}{6.4} & \multirow{3}{*}{$t+4,2,2,3,2^{[t]}, 3,2^{[s-1]}$} & $r=2 t+5$ & \multirow{3}{*}{5} \\
\hline & & $p=\left(r^{2}-2 r-1\right)(s+1)-2 r+5$ & \\
\hline & & $q=r^{2}(s+1)-2 r+1$ & \\
\hline & \multirow{2}{*}{$6,3,2,2$} & $p=25$ & \multirow{2}{*}{ 1B } \\
\hline & & $q=32$ & \\
\hline & \multirow{2}{*}{$5,4,2,2$} & $p=27$ & \multirow{2}{*}{ 1B } \\
\hline & & $q=37$ & \\
\hline \multirow{3}{*}{6.5} & \multirow{3}{*}{$3,2,2,4,2^{[t-1]}$} & $r=3$ & \multirow{3}{*}{5} \\
\hline & & $p=2 t+1$ & \\
\hline & & $q=9 t+4$ & \\
\hline \multirow{2}{*}{7.9} & \multirow{2}{*}{$3,3,3,3$} & $p=13$ & \multirow{2}{*}{ Sporadic } \\
\hline & & $q=34$ & \\
\hline \multirow{6}{*}{7.10} & \multirow{3}{*}{$4,2^{[s-1]}, 3,3,2, s+3,2^{[t-1]}$} & $r=-3-2 s$ & \multirow{3}{*}{4} \\
\hline & & $p=2 r^{2} t-4 r+1$ & \\
\hline & & $q=t(2 r+1)^{2}-8 r-6$ & \\
\hline & \multirow{3}{*}{$5,3,2,3,2^{[t-1]}$} & $r=-3$ & \multirow{3}{*}{4} \\
\hline & & $p=18 t+13$ & \\
\hline & & $q=25 t+18$ & \\
\hline \multirow{3}{*}{7.11} & \multirow{3}{*}{$3,2^{[s-1]}, 4,3, s+2,3,2^{[t-1]}$} & $r=-5-4 s$ & \multirow{3}{*}{2} \\
\hline & & $p=(-4 r-2) t-2 r+3$ & \\
\hline & & $q=r^{2} t+\frac{1}{2}\left(r^{2}-2 r+1\right)$ & \\
\hline \multirow{2}{*}{7.19} & \multirow{2}{*}{$4,2,3,2$} & $p=11$ & \multirow{2}{*}{ Sporadic } \\
\hline & & $q=19$ & \\
\hline \multirow{6}{*}{7.20} & \multirow{3}{*}{$3,2^{[s-1]}, 3,3,2, s+3,3,2^{[t-1]}$} & $r=7+4 s$ & \multirow{3}{*}{2} \\
\hline & & $p=(4 r+2) t+2 r+5$ & \\
\hline & & $q=r^{2} t+\frac{1}{2}\left(r^{2}+2 r-1\right)$ & \\
\hline & \multirow{3}{*}{$4,3,2,3,3,2^{[t-1]}$} & $r=7$ & \multirow{3}{*}{2} \\
\hline & & $p=30 t+19$ & \\
\hline & & $q=49 t+31$ & \\
\hline
\end{tabular}

In this table, $v_{[a, b]}$ means $v_{a}+v_{a+1}+\cdots+v_{b}$ for $a<b$. All vertex bases are presented in the form $\left\{x_{1}, \cdots, x_{n}\right\}$. The parameters $s, t \geq 0$ unless otherwise stated. A superscript ${ }^{[-1]}$ at an element in the sequence of vertex norms means that the sequence is truncated at this element and the element preceding it. For example, the sequence $\left\{3,2^{[s-1]}, 4,3, s+2,3,2^{[t-1]}\right\}$ becomes $\left\{3,2^{[s-1]}, 4,3, s+2\right\}$ when $t=0$. 


\section{REFERENCES}

[AK80] Selman Akbulut and Robion Kirby, Branched covers of surfaces in 4-manifolds, Math. Ann. 252 (1980), 111-131.

$\left[\mathrm{BHM}^{+} 16\right]$ William Ballinger, Chloe C. Hsu, Wyatt Mackey, Yi Ni, Tynan Ochse, and Faramarz Vafaee, The prism manifold realization problem, Preprint, arXiv:1612.04921, 2016.

[BK] John Berge and Sungmo Kang, The hyperbolic $P / P, P / S F_{d}$, and $P / S F_{m} k n o t s$ in $S^{3}$, Preprint.

[Don83] Simon Donaldson, An application of gauge theory to four-dimensional topology, J. Differential Geom. 18 (1983), no. 2, 279-315.

[Gre13] Joshua E. Greene, The lens space realization problem, Ann. of Math. (2) 177 (2013), no. 2, 449-511.

[Gre15] L L-space surgeries, genus bounds, and the cabling conjecture, J. Differential Geom. 100 (2015), no. 3, 491-506.

[Mon73] José M. Montesinos, Seifert manifolds that are ramified two-sheeted cyclic coverings, Bol. Soc. Mat. Mexicana (2) 18 (1973), 1-32.

[OS03] Peter Ozsváth and Zoltán Szabó, Absolutely graded Floer homologies and intersection forms for four-manifolds with boundary, Adv. Math. 173 (2003), no. 2, 179-261.

[OS04a] _ Holomorphic disks and knot invariants, Adv. Math. 186 (2004), no. 1, 58-116.

[OS04b] Holomorphic disks and three-manifold invariants: properties and applications, Ann. of Math. (2) 159 (2004), no. 3, 1159-1245.

[OS05a] _ On knot Floer homology and lens space surgeries, Topology 44 (2005), no. 6, 1281-1300.

[OS05b] On the Heegaard Floer homology of branched double-covers, Adv. Math. 194 (2005), no. 1, $1-33$.

Department of Mathematics, California Institute of Technology, Pasadena, CA 91125

E-mail address: wballing@caltech.edu, yini@caltech.edu, tochse@caltech.edu, vafaee@caltech.edu 